\title{
Measurement of the $k_{\mathrm{t}}$ splitting scales in $Z \rightarrow \ell \ell$ events in $p p$ collisions at $\sqrt{s}=8 \mathrm{TeV}$ with the ATLAS detector
}

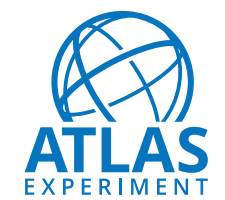

\section{The ATLAS collaboration}

\section{E-mail: atlas.publications@cern.ch}

ABSTRACT: A measurement of the splitting scales occuring in the $k_{\mathrm{t}}$ jet-clustering algorithm is presented for final states containing a $Z$ boson. The measurement is done using $20.2 \mathrm{fb}^{-1}$ of proton-proton collision data collected at a centre-of-mass energy of $\sqrt{s}=8 \mathrm{TeV}$ by the ATLAS experiment at the LHC in 2012. The measurement is based on chargedparticle track information, which is measured with excellent precision in the $p_{\mathrm{T}}$ region relevant for the transition between the perturbative and the non-perturbative regimes. The data distributions are corrected for detector effects, and are found to deviate from state-of-the-art predictions in various regions of the observables.

KEYwords: Hadron-Hadron scattering (experiments)

ArXiv EPrint: 1704.01530 


\section{Contents}

1 Introduction 1

2 ATLAS detector 3

3 Event selection $\quad 4$

3.1 Object reconstruction and event selection at detector level 4

3.2 Particle-level selection and phase-space definition 5

4 Monte Carlo simulation $\quad 5$

4.1 Samples of simulated events 5

$\begin{array}{lll}4.2 & \text { Theoretical predictions } & 6\end{array}$

5 Analysis method $\quad 7$

$\begin{array}{ll}5.1 \text { Background estimation } & 7\end{array}$

5.2 Unfolding 8

$\begin{array}{llr}5.3 & \text { Systematic uncertainties } & 8\end{array}$

6 Results 10

$\begin{array}{llr}7 & \text { Conclusions } & 15\end{array}$

A Results for the electron channel with $R=1.0 \quad 16$

B Results for the muon channel with $R=0.4$ and $R=1.0 \quad 18$

$\begin{array}{ll}\text { The ATLAS collaboration } & 24\end{array}$

\section{Introduction}

A collimated spray of particles arising from a cascade of strong interactions is commonly referred to as a hadronic jet. Jet production in association with other final-state particles can constitute signal as well as an important background process in many of the precision measurements and new-physics searches conducted at CERN's Large Hadron Collider (LHC). A good understanding of processes initiated by strong interactions is therefore crucial.

Jet production in association with a leptonically decaying heavy gauge boson $(V=$ $W, Z)$ allows effects of the strong interaction to be studied in a relatively clean environment. Good progress has been made in recent years towards higher-order calculations of these processes in quantum chromodynamics (QCD), e.g. fixed-order calculations of high jet multiplicities at next-to-leading order (NLO) $[1,2]$ and the inclusive as well as $V+1$-jet processes at next-to-next-to-leading order (NNLO) [3]. Furthermore, new methods have 
been published for matching NLO $V+$ multijet predictions with the parton shower in a merged sample [4-6], or to match NNLO calculations to a parton shower for the inclusive processes $[7,8]$. Comparisons of precision measurements to these accurate predictions are a powerful means by which to study aspects of QCD.

While properties of the jets can be studied directly using the jet momenta, a complementary approach is taken in this paper by studying the jet production rates at different resolution scales. To this end, splitting scales of jets are constructed using an infraredsafe clustering algorithm based on sequential combination of the input momenta. In this analysis the $k_{\mathrm{t}}$ algorithm $[9,10]$ is used, with distance measures defined for every iteration as follows:

$$
\begin{aligned}
& d_{i j}=\min \left(p_{\mathrm{T}, i}^{2}, p_{\mathrm{T}, j}^{2}\right) \times \frac{\Delta R_{i j}^{2}}{R^{2}}, \\
& d_{i b}=p_{\mathrm{T}, i}^{2},
\end{aligned}
$$

where the transverse momentum $p_{\mathrm{T}}$ carries an index corresponding to the $i^{\text {th }}$ and $j^{\text {th }}$ constituent momentum in the input list, for all possible permutations of $i$ and $j$ in the given clustering step. The input momenta separation $\Delta R_{i j}$ is defined in terms of the rapidity $y$ and the azimuthal angle $\phi$ via the relation $\left(\Delta R_{i j}\right)^{2}=\left(y_{i}-y_{j}\right)^{2}+\left(\phi_{i}-\phi_{j}\right)^{2}$. The index $b$ denotes the beam line and the parameter $R$ governs the average cone size in $y-\phi$ space around the jet axis. For a given iteration of the algorithm in which the number of input momenta drops from $k+1$ to $k$, the associated squared splitting scale $d_{k}$ is given by the minimum of all the $d_{i j}$ and $d_{i b}$ scales defined for that iteration step:

$$
d_{k}=\min _{i, j}\left(d_{i j}, d_{i b}\right)
$$

If this minimum is a $d_{i j}$, the $i^{\text {th }}$ and $j^{\text {th }}$ momenta in the input list are replaced by their combination. If the minimum is a $d_{i b}$, the $i^{\text {th }}$ momentum is removed from the input collection and is declared a jet. The index $k$ defines the order of the splitting scale, with $k=0$ being the last iteration step before the algorithm terminates. Hence the zeroth-order splitting scale, $\sqrt{d_{0}}$, corresponds to the $p_{\mathrm{T}}$ of the leading $k_{\mathrm{t}}$-jet, and one can regard the $N^{\text {th }}$ splitting scale, $\sqrt{d_{N}}$, as the distance measure at which an $N$-jet event is resolved as an $(N+1)$-jet event. The steps of a $k_{\mathrm{t}}$ clustering sequence using three input momenta are illustrated in figure 1.

In this paper, measurements of differential distributions of the splitting scales occurring in the $k_{\mathrm{t}}$ clustering algorithm using charged-particle tracks in events with $Z+$ jets are presented. The aim is to constrain the theoretical modelling of strong-interaction effects, and charged-particle tracks are used instead of calorimeter cells to reduce the systematic uncertainties of the measurements significantly. In addition to these primary results using only charged-particle information, less precise extrapolated results including neutral particles are also provided to allow comparisons to fixed-order calculations.

The measurements are performed independently in the $Z \rightarrow e^{+} e^{-}$and $Z \rightarrow \mu^{+} \mu^{-}$ decay channels as well as for jet-radius parameters of $R=0.4$ and $R=1.0$ in each decay channel. The presented analysis is complementary to the ATLAS measurement of the $k_{\mathrm{t}}$ splitting scales in $W+$ jets events at $\sqrt{s}=7 \mathrm{TeV}[11]$. 


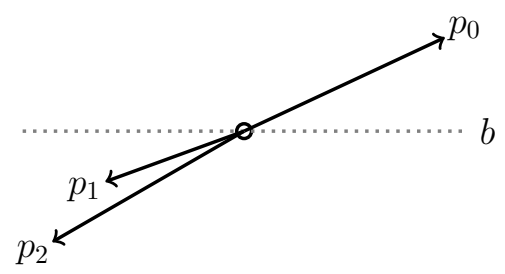

(a) Step 1 .

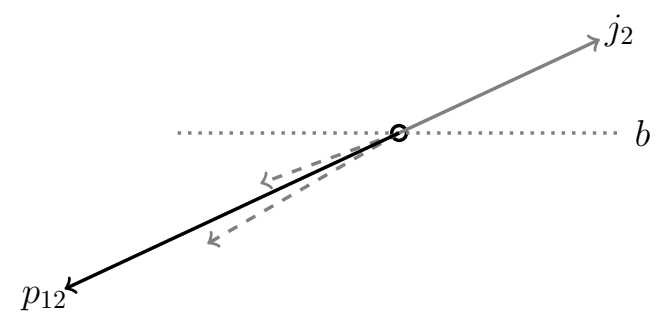

(c) Step 3 .

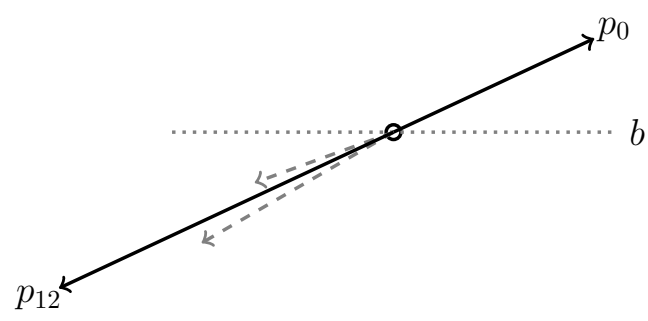

(b) Step 2.

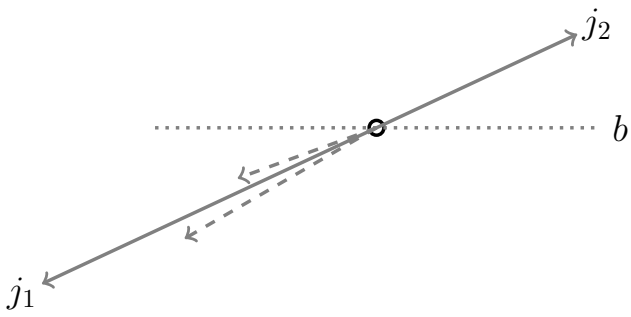

(d) Step 4.

Figure 1. Simplified illustration of the $k_{\mathrm{t}}$ clustering algorithm, starting with three input momenta $p_{0}, p_{1}$ and $p_{2}$ (step 1). The dotted line labelled $b$ represents the beam line. In step 2 , the minimum distance measure is the one between two input momenta $p_{1}$ and $p_{2}$, so that the two input momenta are replaced by their vector combination. In step 3 , the minimum distance measure is between the $p_{0}$ and the beam line, so that $p_{0}$ is declared a jet $\left(j_{2}\right)$ and removed from the input list. Finally in step 4 , there is only the combined input momentum $p_{12}$ left and so it will be declared a jet $\left(j_{1}\right)$ and the algorithm terminates.

\section{ATLAS detector}

The ATLAS detector is described in detail in ref. [12]. Tracks and interaction vertices are reconstructed with the inner detector (ID) tracking system, consisting of a silicon pixel detector, a silicon microstrip detector (SCT) and a transition radiation tracker. The ID is immersed in a $2 \mathrm{~T}$ axial magnetic field, providing charged-particle tracking in the pseudorapidity range $|\eta|<2$. . $^{1}$ The ATLAS calorimeter system provides fine-grained measurements of shower energy depositions over a wide range of $\eta$. An electromagnetic liquid-argon sampling calorimeter covers the region $|\eta|<3.2$ and is divided into a barrel part $(|\eta|<1.475)$ and an endcap part $(1.375<|\eta|<3.2)$. The hadronic barrel calorimeter $(|\eta|<1.7)$ consists of steel absorbers and active scintillator tiles. The hadronic endcap calorimeter $(1.5<|\eta|<3.2)$ and forward electromagnetic and hadronic calorimeters $(3.1<|\eta|<4.9)$ use liquid argon as the active medium. The muon spectrometer comprises separate trigger and high-precision tracking chambers measuring the deflection

\footnotetext{
${ }^{1}$ ATLAS uses a right-handed coordinate system with its origin at the nominal interaction point (IP) in the centre of the detector and the $z$-axis along the beam pipe. The $x$-axis points from the IP to the centre of the LHC ring, and the $y$-axis points upward. Cylindrical coordinates $(r, \phi)$ are used in the transverse plane, $\phi$ being the azimuthal angle around the $z$-axis. The pseudorapidity is defined in terms of the polar angle $\theta$ as $\eta=-\ln \tan (\theta / 2)$.
} 
of muons in a magnetic field generated by superconducting air-core toroids. The precision chamber system covers the region $|\eta|<2.7$ with three layers of monitored drift tube chambers, complemented by cathode strip chambers in the forward region. The muon trigger system covers the range $|\eta|<2.4$ with resistive plate chambers in the barrel, and thin gap chambers in the endcap regions. A three-level trigger system is used to select events of interest [13]. The Level-1 trigger reduces the event rate to less than $75 \mathrm{kHz}$ using hardware-based trigger algorithms acting on a subset of the available detector information. Two software-based trigger levels then reduce the event rate further to about $400 \mathrm{~Hz}$ using the complete detector information.

\section{Event selection}

The measurement is performed using proton-proton collision data recorded at a centre-ofmass energy of $\sqrt{s}=8 \mathrm{TeV}$. The data were collected between April and December 2012 in data-taking periods where the detector was fully operational, resulting in an integrated luminosity of $20.2 \mathrm{fb}^{-1}$.

\subsection{Object reconstruction and event selection at detector level}

Events containing a dilepton candidate were retained for further analysis using dedicated triggers requiring the presence of two oppositely charged electrons with transverse momentum above $12 \mathrm{GeV}$ or two oppositely charged muons with transverse momenta above $18 \mathrm{GeV}$ and $8 \mathrm{GeV}$ respectively.

Inner detector tracks are selected in the phase-space region $p_{\mathrm{T}}>400 \mathrm{MeV}$ and $|\eta|<2.5$. The track candidates are required to have at least one hit in the pixel detector and at least five SCT hits. A hit in the innermost pixel layer is required in cases where the track candidates have passed through an active region of that layer. The track reconstructed from the hits is then extrapolated and combined with information from the transition radiation tracker [14]. The reduced $\chi^{2}$ of the track fit is required to be less than 3 in order to remove mismeasured tracks or combinatorial background. In order to reject backgrounds stemming from other proton-proton collisions in the same or different bunch crossings (pileup), the transverse and longitudinal impact parameters are required to be $\left|d_{0}\right|<$ $1.0 \mathrm{~mm}$ and $\left|z_{0} \times \sin \theta\right|<0.6 \mathrm{~mm}$ with respect to the primary vertex, respectively. The primary vertex in the event is defined as the collision vertex with the highest sum of squared transverse momenta of the associated ID tracks.

Electron candidates are identified as clusters of energy in the electromagnetic calorimeter which are associated with a corresponding ID track. They are required to have $p_{\mathrm{T}}>25 \mathrm{GeV}$ and $|\eta|<2.47$, excluding the transition regions between the barrel and endcap electromagnetic calorimeters $(1.37<|\eta|<1.52)$. The electron candidates must satisfy a set of medium selection criteria [15] that have been optimised for the high rate of proton-proton collisions per beam crossing observed in the 2012 data. Electron candidates are required to be isolated, meaning that the scalar sum of the $p_{\mathrm{T}}$ of those tracks within $\Delta R=0.2$ around the electron track is required to be less than $13 \%$ of the $p_{\mathrm{T}}$ of the electron. (The definition of $\Delta R$ is the same as for the $k_{\mathrm{t}}$ algorithm, except that it makes 
use of pseudorapidity rather than rapidity.) Impact parameter requirements are imposed to ensure that the electron candidates originate from the primary vertex.

Muon candidates are reconstructed using the combined muon algorithm [16], which involves matching and combining ID tracks with tracks in the muon spectrometer. The muon candidates are required to have $p_{\mathrm{T}}>25 \mathrm{GeV}$ and $|\eta|<2.4$. Muon track quality requirements are imposed to suppress backgrounds, along with impact parameter requirements to ensure that the muon candidates originate from the primary vertex. Muon candidates are also required to be isolated, meaning that the scalar sum of the $p_{\mathrm{T}}$ of those tracks within $\Delta R=0.2$ around the muon track (using pseudorapidity again) is required to be less than $10 \%$ of the $p_{\mathrm{T}}$ of the muon.

A $Z$-boson candidate is selected by requiring exactly two opposite-charge same-flavour leptons (electrons or muons) and requiring the invariant mass of the dilepton system to satisfy $71 \mathrm{GeV}<m_{\ell \ell}<111 \mathrm{GeV}$. The momenta of all selected ID tracks - apart from the two lepton tracks - are then passed into the $k_{\mathrm{t}}$ clustering algorithm introduced in section 1 to construct the splitting scales.

\subsection{Particle-level selection and phase-space definition}

Particle-level predictions are obtained using final-state objects with a mean decay length $(c \tau)$ longer than $10 \mathrm{~mm}$. Leptons are defined at the dressed level, i.e. they are given by the four-momentum combination of the respective lepton (an electron or a muon) and all nearby photons within a cone of size $\Delta R=0.1$ centred on the lepton. Electrons are required to pass $|\eta|<2.47$, excluding the transition region between the barrel and end cap electromagnetic calorimeters $1.37<|\eta|<1.52$, whilst muons are selected with $|\eta|<2.4$. Furthermore, a transverse momentum requirement of $p_{\mathrm{T}}>25 \mathrm{GeV}$ is imposed for either lepton flavour.

Events are required to contain a $Z$-boson candidate, defined as exactly two oppositely charged, same-flavour leptons (electrons or muons) with a dilepton invariant mass of $71 \mathrm{GeV}<m_{\ell \ell}<111 \mathrm{GeV}$.

All charged final-state particles with $p_{\mathrm{T}}>400 \mathrm{MeV}$ and $|\eta|<2.5$ - excluding the selected leptons - serve as input to the $k_{\mathrm{t}}$ clustering algorithm to construct the splittingscale observables. The measurement is performed twice, using jet-radius parameters of 0.4 and 1.0 respectively. This allows studies of the resolution scales of both narrow and broad jets, which have different sensitivity to the hadronisation and underlying-event modelling.

\section{Monte Carlo simulation}

\subsection{Samples of simulated events}

Signal event samples for $Z \rightarrow e^{+} e^{-}$and $Z \rightarrow \mu^{+} \mu^{-}$production in association with jets (QCD $Z+$ jets) were generated in order to correct the data for detector effects in an unfolding procedure and to estimate systematic uncertainties. The samples were produced using the SherPa v1.4.3 and Powheg-Box [17-19] (SVN revision r1556) event generators. The SHERPA samples are based on matrix elements with up to four additional hard 
emissions at leading order (LO), using parton distribution functions (PDFs) from the CT10 set [20], which were matched and merged to the SHERPA parton shower using the set of tuned parameters developed by the Sherpa authors. The Powheg-Box samples (hereafter referred to as the Powheg samples) use the CT10 PDF set and are passed through Pythia v8.175 and subsequently through PHOTOS++ [21] for parton showering and radiative quantum-electrodynamical corrections, respectively.

$Z \rightarrow \tau^{+} \tau^{-}$as well as $W+$ jets production were generated with SHERPA using the MENLOPS prescription [22] to merge the results of inclusive $Z \rightarrow \tau^{+} \tau^{-}$and $W \rightarrow \ell \nu$ calculations performed at NLO accuracy with the LO multi-leg prediction. All QCD $V+$ jets samples are normalised using an inclusive NNLO cross section [23].

Processes involing matrix elements with up to one additional parton emission for electroweak $Z+$ jets production (including all diagrams with three electroweak couplings at tree level) were simulated using SHERPA. The background contribution from $W W$ production with both bosons decaying leptonically is estimated using PowhEG+PythiA 8.

Background contributions stemming from top-quark interactions $(t \bar{t}, t$-channel single top and $W t$ ) were generated using PowHEG with the CT10 PDF set in the hard scattering in conjunction with PYTHIA v6.427 [24] for parton showering and hadronisation using the CTEQ6L1 [25] PDF set and the corresponding Perugia 2011C [26] set of tuned parameters. The hdamp parameter, which controls the $p_{\mathrm{T}}$ of the first additional emission beyond the Born configuration, is set to the mass of the top quark. The $t \bar{t}$ sample is normalised using a NNLO calculation in QCD including resummation of next-to-next-to-leading logarithmic (NNLL) soft gluon terms [27], while the single-top samples are normalised using an approximate NNLO calculation including NNLL-accurate resummation of soft gluon terms [28-30].

The Monte Carlo event samples mentioned above were passed through GEANT4 [31, 32] for a full simulation [33] of the ATLAS detector and are reconstructed with the same analysis chain as used for the measured data. Pileup is simulated with PYTHIA v8.175 [34] using the A2 [35] set of tuned parton shower parameters and the MSTW2008lo [36] set of parton distribution functions.

\subsection{Theoretical predictions}

In addition to the samples of fully simulated events, additional particle-level predictions were generated to provide a state-of-the-art comparison to the unfolded measurements.

Predictions for $Z$ boson production in association with jets are obtained using the Sherpa v2.2.1 generator [37]. Matrix elements are calculated for up to two additional parton emissions at NLO accuracy and up to four additional parton emissions at LO accuracy using the Comix [38] and OpenLoops [39] matrix element generators, and merged with the SHERPA parton shower [40] which is based on Catani-Seymour subtraction terms. The merging of multi-parton matrix elements with the parton shower is achieved using an improved CKKW matching procedure [41, 42], which is extended to NLO accuracy using the MEPS@NLO prescription [4]. The PDFs are provided by the NNPDF3.0nnlo set [43] and the dedicated set of tuned parton shower parameters developed by the SHERPA authors is used. 


\begin{tabular}{|lrrrr|}
\hline & \multicolumn{2}{c}{$Z \rightarrow e^{+} e^{-}$} & \multicolumn{2}{c|}{$Z \rightarrow \mu^{+} \mu^{-}$} \\
Process & Events & Contribution [\%] & Events & Contribution [\%] \\
\hline QCD $Z+$ jets & 5090000 & $98.93 \%$ & 7220000 & $99.40 \%$ \\
Multijet & 42000 & $0.81 \%$ & 25000 & $0.34 \%$ \\
Electroweak $Z+$ jets & 5350 & $0.10 \%$ & 7340 & $0.10 \%$ \\
Top quarks & 6190 & $0.12 \%$ & 8440 & $0.12 \%$ \\
$W(W)$ & 1100 & $0.02 \%$ & 1460 & $0.02 \%$ \\
$Z \rightarrow \tau^{+} \tau^{-}$ & 1100 & $0.02 \%$ & 1700 & $0.02 \%$ \\
\hline Total expected & 5150000 & $100.00 \%$ & 7260000 & $100.00 \%$ \\
\hline Total observed & 5196858 & & 7349195 & \\
\hline
\end{tabular}

Table 1. Observed and expected numbers of events in the electron and the muon channels. The signal as well as the electroweak $Z+$ jets, $W W, W+$ jets, $t \bar{t}$ and single-top background rates are estimated using dedicated Monte Carlo samples. The multijet background is estimated using a data-driven technique.

Predictions for $Z+$ jets production at NNLO were provided by the DY@NNLOPS authors [8]. The calculations are obtained using DYNNLO [44, 45] along with the multiscale improved NLO (MiNLO) prescription [46] for the scale choices as implemented in the PowheG-Box package [19, 47]. For the hard scattering the PDF4LHC15nnlo PDF set [48] is used, and parton showering is provided by PYTHIA v8.185 using the Monash [49] set of tuned parameters.

\section{Analysis method}

Distributions of the $k_{\mathrm{t}}$ splitting scales up to the seventh order were constructed from track momenta selected according to section 3.1.

\subsection{Background estimation}

Events from the signal process and the background contributions from $Z \rightarrow \tau^{+} \tau^{-}, W+$ jets, top-quark pair and single top quark production as well as diboson and electroweak $Z+$ jets production are obtained by applying the analysis chain to the dedicated simulated samples introduced in section 4.1. The multijet background can also contribute if two jets are misidentified as leptons or if they contain leptons from $b$ - or $c$-hadron decays. A multijetenhanced sample is obtained from the data by reversing some of the lepton identification criteria. A two-component template fit to the dilepton invariant mass spectrum is then employed to determine the normalisation in the fiducial measurement region, using the multijet template and a template formed from all other processes. Table 1 shows the estimated composition in each lepton channel. The relative sizes of the various background contributions are also illustrated in figure 2 , which shows the $\sqrt{d_{1}}$ distribution at the detector level in both the electron and the muon channel. The purity of the signal is close to $99 \%$. 

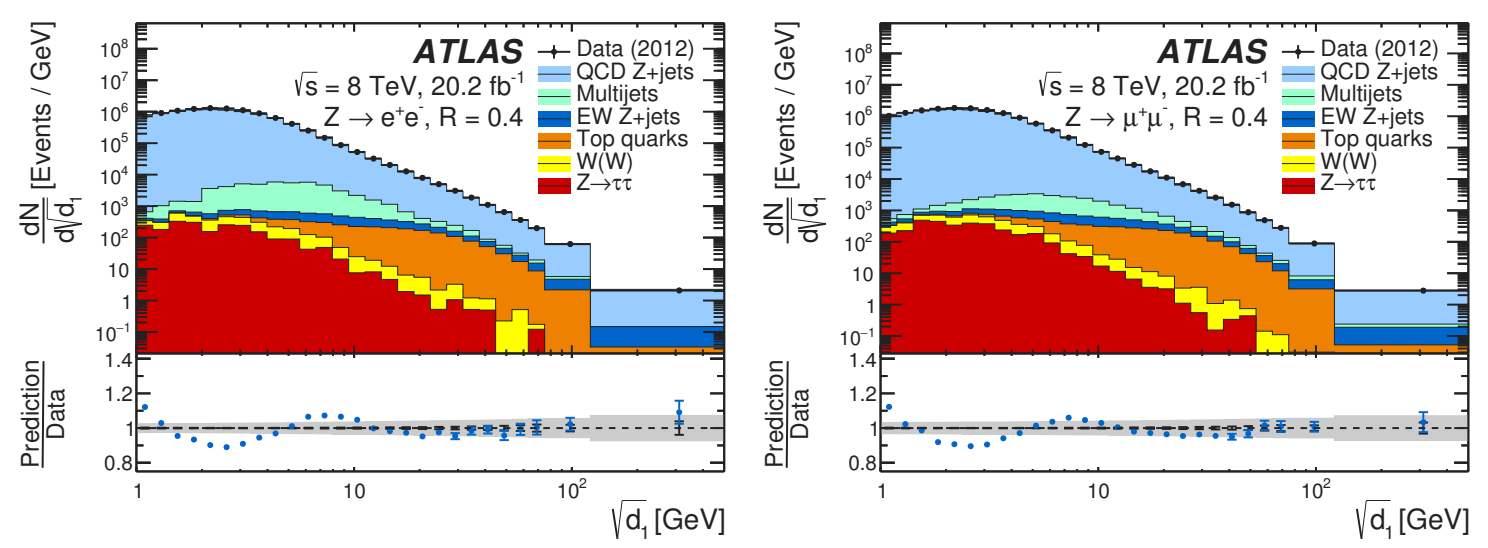

Figure 2. Detector-level splitting scale distributions in the electron and muon channels using the jet-radius parameter $R=0.4$. The lower panel shows the ratio of the combined Monte Carlo predictions to the data. The size of the error bars reflects the statistical uncertainty for predictions and data, while the combined experimental and systematic data uncertainty is indicated by a grey band around unity.

\subsection{Unfolding}

The estimated number of background events is subtracted from the data in each bin of a given distribution. The background-subtracted data are then unfolded back to the particle level using an iterative procedure based on Bayes' theorem [50, 51], which makes use of an unfolding prior that is updated at each iteration. In the first iteration, the nominal particlelevel predictions from SHERPA are used as the unfolding prior. The detector resolution causes bin-to-bin migrations, which are corrected for using a detector response matrix in the unfolding procedure. Acceptance and efficiency losses in the fiducial measurement region as well as fake contributions (e.g. due to pileup) are also accounted for. The unfolded bin values are found to converge after five iterations in most cases. The statistical uncertainty in data and simulation is estimated using pseudoexperiments.

In addition to the nominal charged-particle-level results ("charged-only"), the unfolding procedure is repeated using particle-level predictions that include the neutral particles as well, thereby extrapolating the data to a particle-level including all particles ("charged+neutral"). The requirements on the $Z$-boson candidate and the particles entering the clustering sequence remain identical to those of the charged-only analysis. These extrapolated results are provided for the benefit of theoretical calculations which cannot account for hadronisation processes, and depend strongly on the modelling of hadronisation processes in the generator used during the unfolding. Since the extrapolation and the corresponding uncertainty estimate could change with other hadronisation models in the future, the "charged-only" measurements should be considered the primary results of this publication.

\subsection{Systematic uncertainties}

The systematic variations outlined below are propagated through the unfolding procedure by creating a new response matrix constructed from the simulation after reweighting, 
smearing or shifting the relevant event weights or objects. The shift in the unfolded spectrum is symmetrised and taken as a systematic uncertainty in the final result. The binning is chosen to be uniform in logarithmic space, with some bins merged towards the tails of the distributions to compensate for statistical fluctuations in the data.

Experimental uncertainties arise from the lepton-based and luminosity systematic uncertainties, the pileup modelling as well as the track reconstruction efficiency. The systematic uncertainties in the lepton reconstruction, identification, isolation and trigger efficiencies, as well as the lepton momentum scale and resolution, are defined in refs. $[15,16]$. The total impact of the lepton-based systematic uncertainties on the final results is typically $1 \%$ or less. The uncertainty in the integrated luminosity is $1.9 \%$, as determined from beam separation scans [52] performed in November 2012. The difference in the pileup profile between the detector-level simulation and the data is corrected for by reweighting the simulation to match the average number of proton-proton collisions observed in the data. The impact of the remaining mismodelling on the measurement is found to be at most $1 \%$, which is assigned as a systematic uncertainty. The efficiency to reconstruct an ID track depends on the ID material distribution. A corresponding uncertainty is derived by comparing the nominal simulation to a dedicated simulation using a distorted ID geometry and a $15 \%$ increase in the material budget. The resulting systematic uncertainty associated with the track reconstruction efficiency is typically at the level of $5 \%$ but can rise to $10 \%$ in the tails of the distributions, in particular for higher-order splitting scales. Uncertainties associated with the track momentum are found to be negligible for the range of track momenta probed in this measurement. As a cross-check, splitting scales are also constructed in data and simulation using tracks that do not pass the nominal longitudinal impact parameter requirements in order to assess the modelling of pileup and fake tracks in the simulation. Good agreement is observed between data and simulation, with the data being generally described to within $10 \%$. The differences are covered by the assigned uncertainties, which are larger in that region due to the larger fraction of fake and pileup tracks, and so no additional systematic uncertainties in the pileup modelling or fake-track rates are assigned.

A data-driven uncertainty associated with the imperfect modelling of the unfolded observables at the detector level is obtained by reweighting the simulation such that the level of agreement between the detector-level distributions and the data is improved before performing the unfolding procedure. The reweighting is applied at the particle level using the ratio of the distributions in data and detector-level simulation and results in a systematic uncertainty of at most $5 \%$. An algorithmic uncertainty associated with the number of iterations chosen in the unfolding procedure is taken to be the difference between the results unfolded using ten iterations and the nominal results obtained using five unfolding iterations. The algorithmic unfolding uncertainty is at the subpercent level.

An uncertainty due to the choice of generator used to unfold the data, which is the dominant systematic uncertainty, is obtained by replacing the nominal SHERPA prediction in the full analysis chain with a prediction from Powheg+Pythia 8, which uses a very different parton shower and hadronisation model compared to the nominal generator. The difference between the results unfolded with the nominal predictions and the alternative 
predictions is then symmetrised and assigned as an uncertainty in the choice of unfolding prior. This uncertainty estimate might be reduced by reweighting both SHERPA and Powheg+Pythia 8 to the experimental data at reconstruction level before constructing the response matrices. Since it is not clear that this would still cover the full impact of non-perturbative effects on the unfolding, no such reweighting is applied when assessing the unfolding-prior uncertainty.

The extrapolated "charged+neutral" results are also obtained using the generator combinations described above as unfolding priors, providing an estimate of the extrapolation uncertainty.

To cover further systematic effects of the unfolding algorithm on the extrapolated results, an MC-closure uncertainty was derived by unfolding Powheg+Pythia 8 from detector level to the charged+neutral level using response matrices generated from the ShERPA samples. The difference with respect to the direct charged+neutral predictions from Powheg+Pythia 8 is then added as an MC-based closure uncertainty in analogy to the data-driven reweighting uncertainty described above.

The breakdown of the corresponding systematic uncertainties can be found in figure 3 . In the breakdowns, the total uncertainty shown is the full quadrature sum of all the individual statistical and systematic uncertainty components. This includes a constant $\pm 1.9 \%$ uncertainty in the luminosity estimate, which is omitted from the breakdown for clarity. The curve labelled 'experimental' contains the tracking and pileup uncertainties as well as the systematic uncertainties associated with the $Z$-boson candidate selection. The curve labelled 'unfolding' contains the uncertainties associated with the unfolding procedure, namely the unfolding closure uncertainty, the uncertainty associated with the number of iterations as well as the unfolding-prior uncertainty.

\section{Results}

In this analysis, distributions for eight splitting scales, $\sqrt{d_{0}} \ldots \sqrt{d_{7}}$, are obtained for all combinations of

- two lepton flavours, $Z \rightarrow e^{+} e^{-}$and $Z \rightarrow \mu^{+} \mu^{-}$,

- two radius parameters in the jet algorithm, $R=0.4$ and $R=1.0$, and

- two particle-level definitions: "charged-only" and "charged+neutral", as introduced in section 5.2.

A full set considering all splitting scales is shown only for the electron-channel analysis with $R=0.4$ in figure 4 , while other combinations can be found in appendices $\mathrm{A}$ and $\mathrm{B}$.

The measured differential cross sections are compared to state-of-the-art predictions obtained from SHERPA ('MEPS@NLO') and DY@NNLO+PowhEG+Pythia 8 ('NNLOPS'). Details of the respective generator setups are given in section 4.2. PDF uncertainties for the MEPS@NLO prediction are estimated with the NNPDF3.0nnlo [43] replicas using LHAPDF [53]. They are combined with an uncertainty estimate based on 

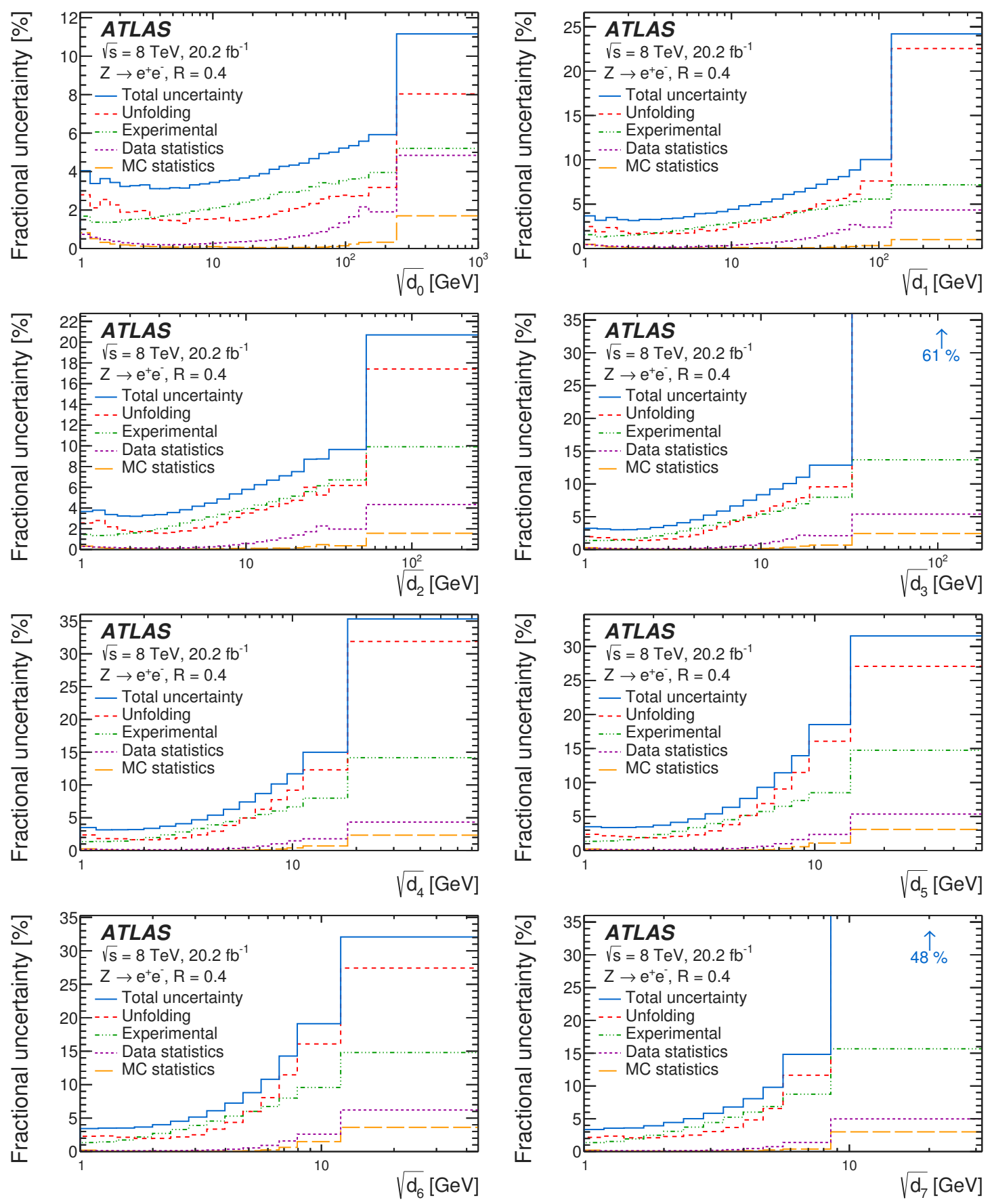

Figure 3. Breakdowns of the total systematic uncertainty into its contributions in the electron channel using the jet-radius parameter $R=0.4$. The total uncertainty shown is the full quadrature sum of all the individual statistical and systematic uncertainty components, including a constant $\pm 1.9 \%$ uncertainty in the luminosity estimate. 
7-point scale variations, i.e. all combinations of factors of $0.5,1$ and 2 in the factorisation and renormalisation scales are taken into account except the opposite combinations of $(0.5,2)$ or $(2,0.5)$. The scale uncertainty envelope for the NNLOPS prediction is obtained from 21-point scale variations using the prescription described in ref. [8].

Neither of the generators provides a fully satisfactory description of the experimental data. In the ratio plots of the lower-order splitting scales it is seen that both predictions underestimate the cross section in the peak region at values of around $3 \mathrm{GeV}$ by typically 10-20\%, but are consistent with the data at $10 \mathrm{GeV}$. At higher values the MEPS@NLO prediction agrees well with the data while the NNLOPS prediction systematically overestimates the cross section. Both overshoot the data significantly in the region close to $1 \mathrm{GeV}$. The level of agreement of the NNLOPS predictions in the soft region is improved significantly for the higher-order splitting scales.

The generator uncertainties in these comparisons are only estimated by varying parameters related to the perturbative aspects of the MC generators. In the soft region of the splitting-scale distributions other aspects become relevant, such as hadronisation and multiple parton interactions, and even the parton shower modelling. The discrepancies unveiled by these measurements indicate that the data can provide new input for the tuning of parameters in the non-perturbative stages of event generators.

Figure 5 displays again the first-order splitting scale, $\sqrt{d_{1}}$, to demonstrate the different variations in the analysis due to the choice of lepton flavour, jet-radius parameter and particle level. A detailed breakdown of the systematic uncertainties is included here in the same style as described earlier.

The uncertainties for the extrapolated charged+neutral distributions are increased due to the unfolding procedure, which includes the correction for going from a fiducial phase space using only charged particles to one using all particles. This effect is most significant for low values of the lower-order splitting scales such as $\sqrt{d_{1}}$, where uncertainties grow beyond $10 \%$ compared to less than $5 \%$ in the charged-only measurement. For higher values of the splitting scales the differences between the charged-only and charged + neutral uncertainties are not as large, as this region is not affected much by hadronisation effects. The peak of the extrapolated distributions, when compared to the nominal (charged) distributions, is shifted by roughly $1 \mathrm{GeV}$ in the $R=0.4$ case and by about $2.5 \mathrm{GeV}$ in the $R=1.0$ case. It moves to higher values of the splitting scales as expected given that the jet energies increase with the addition of neutral particles.

The two lepton flavours agree to better than $1 \sigma$, once differences in the fiducial lepton selection are accounted for. The channels are not combined, because data statistics is a subdominant uncertainty. The $R=1.0$ comparisons reveal features similar to the $R=0.4$ case. A full set of distributions and systematic uncertainties in the $R=1.0$ case are shown in appendix A. Distributions of all possible combinations are furthermore provided in HEPDATA [54] along with an associated Rivet [55] routine. 

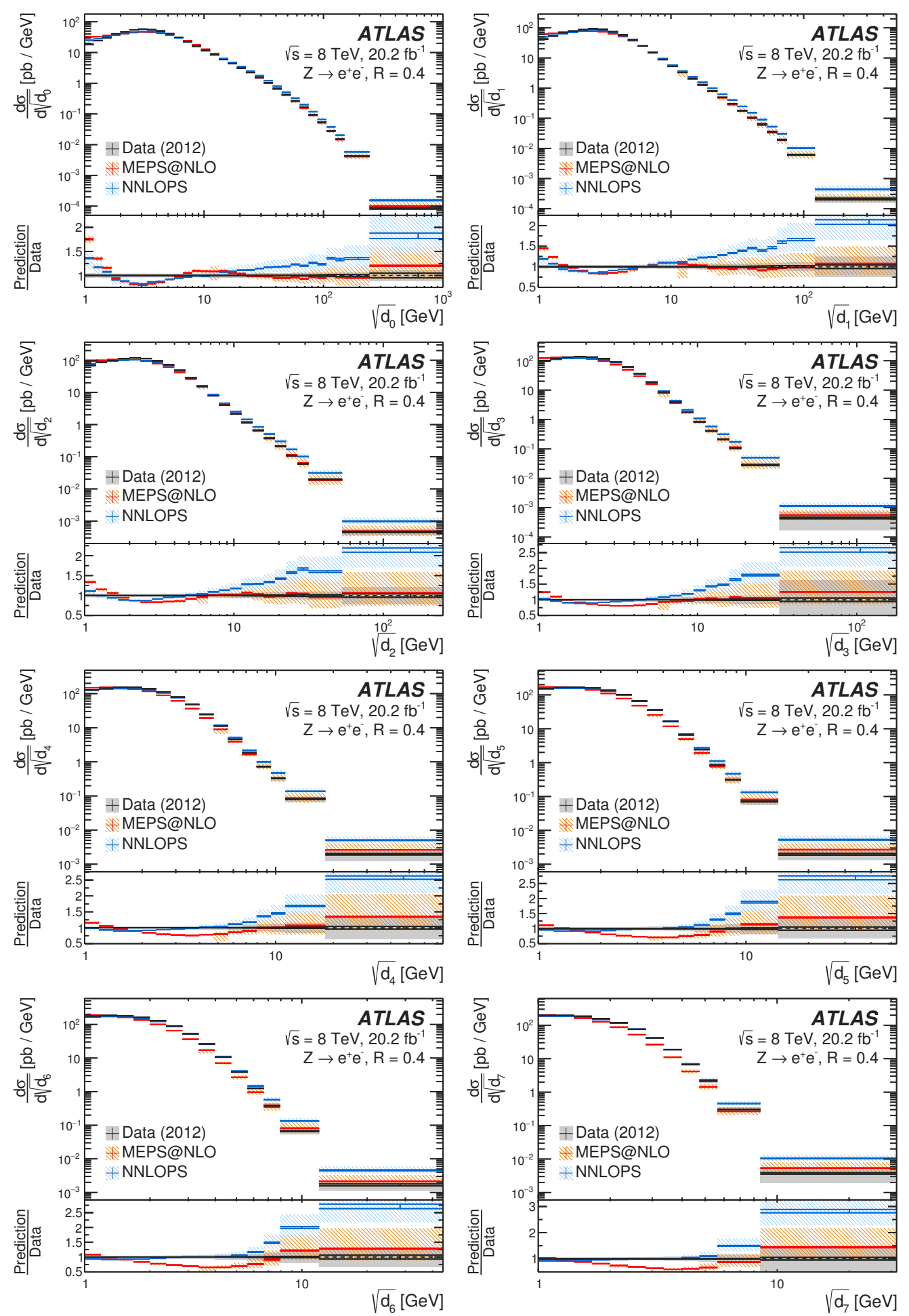

Figure 4. Charged-only distributions for the eight leading splitting scales in the electron channel using the jet-radius parameter $R=0.4$. The size of the error bars reflects the statistical uncertainty, while the combined statistical and systematic uncertainty is indicated by the grey band. Theoretical predictions from SHERPA with NLO multijet merging ("MEPS@NLO") and from Powheg+Pythia 8 with NNLO matching ("NNLOPS") are displayed including error bands for the generator uncertainties. 

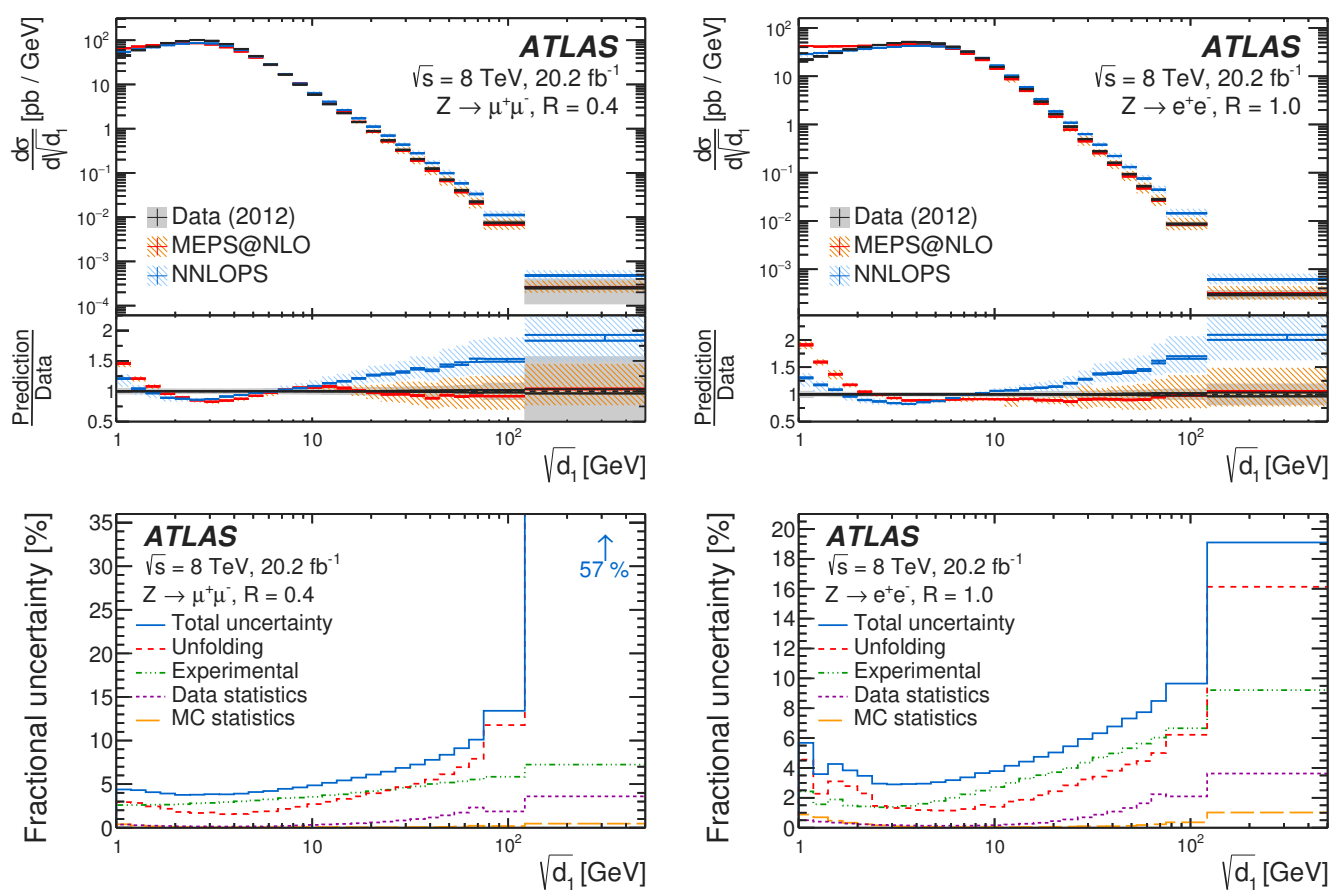

(a) muon, $R=0.4$, charged-only

(b) electron, $R=1.0$, charged-only
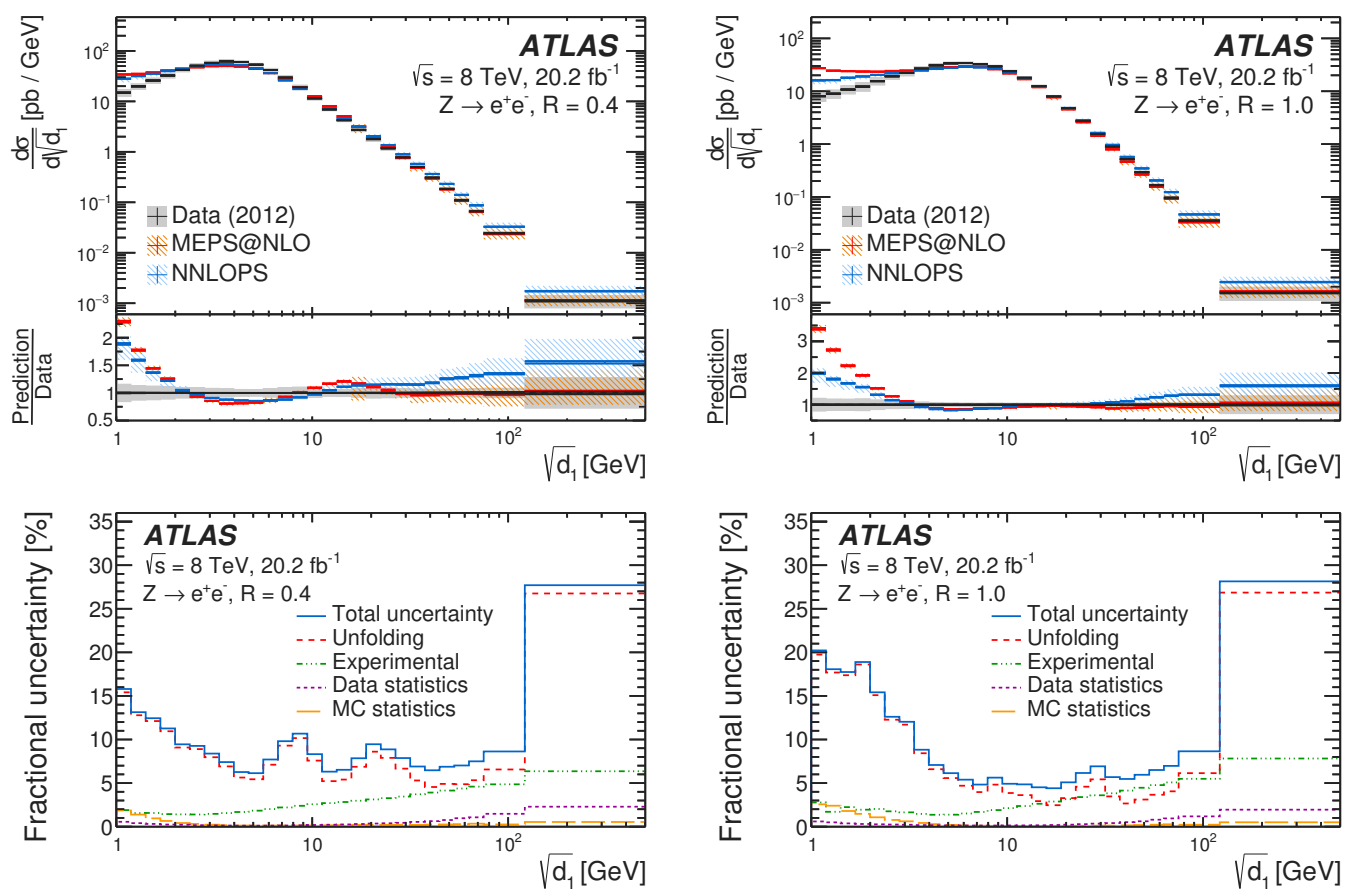

(c) electron, $R=0.4$, charged + neutral

(d) electron, $R=1.0$, charged+neutral

Figure 5. Unfolded $\sqrt{d_{1}}$ splitting scale for four different variations of the analysis as described in the sub-captions. The upper panels show the distributions in the same style as figure 4. The lower panels show breakdowns of the systematic uncertainties in data in the same style as figure 3. 


\section{Conclusions}

Differential cross sections are measured as a function of the splitting scales in the $k_{\mathrm{t}}$ algorithm applied to the hadronic activity in $Z \rightarrow \ell \ell$ events. The measurements use an $8 \mathrm{TeV}$ proton-proton collison data set recorded by the ATLAS detector at the LHC with an integrated luminosity of $20.2 \mathrm{fb}^{-1}$.

Splitting scales up to the seventh order are measured for both the $Z \rightarrow e^{+} e^{-}$and $Z \rightarrow \mu^{+} \mu^{-}$channels and for jet-radius parameters of $R=0.4$ and $R=1$.0. Chargedparticle tracks are used for the analysis.

The measurements are corrected for detector effects. In addition to the charged-particle final state, a Monte Carlo simulation-based extrapolation to the charged- and neutralparticle final state is provided. The final results are compared to two state-of-the-art theoretical predictions, one including NNLO accuracy matrix elements, and a second prediction with multi-leg NLO merging. Significant deviations from data are found for both predictions, in the perturbative as well as the non-perturbative regimes.

The measurements of splitting scales are sensitive to the hard perturbative modelling at high scale values as well as soft hadronic activity at lower values. They thus provide a valuable input complementary to standard jet measurements, in particular in the transition region. With their specific identification of QCD jet evolution they provide means to constrain and potentially tune Monte Carlo event generators.

\section{Acknowledgments}

We thank CERN for the very successful operation of the LHC, as well as the support staff from our institutions without whom ATLAS could not be operated efficiently.

We acknowledge the support of ANPCyT, Argentina; YerPhI, Armenia; ARC, Australia; BMWFW and FWF, Austria; ANAS, Azerbaijan; SSTC, Belarus; CNPq and FAPESP, Brazil; NSERC, NRC and CFI, Canada; CERN; CONICYT, Chile; CAS, MOST and NSFC, China; COLCIENCIAS, Colombia; MSMT CR, MPO CR and VSC CR, Czech Republic; DNRF and DNSRC, Denmark; IN2P3-CNRS, CEA-DSM/IRFU, France; SRNSF, Georgia; BMBF, HGF, and MPG, Germany; GSRT, Greece; RGC, Hong Kong SAR, China; ISF, I-CORE and Benoziyo Center, Israel; INFN, Italy; MEXT and JSPS, Japan; CNRST, Morocco; NWO, Netherlands; RCN, Norway; MNiSW and NCN, Poland; FCT, Portugal; MNE/IFA, Romania; MES of Russia and NRC KI, Russian Federation; JINR; MESTD, Serbia; MSSR, Slovakia; ARRS and MIZŠ, Slovenia; DST/NRF, South Africa; MINECO, Spain; SRC and Wallenberg Foundation, Sweden; SERI, SNSF and Cantons of Bern and Geneva, Switzerland; MOST, Taiwan; TAEK, Turkey; STFC, United Kingdom; DOE and NSF, United States of America. In addition, individual groups and members have received support from BCKDF, the Canada Council, CANARIE, CRC, Compute Canada, FQRNT, and the Ontario Innovation Trust, Canada; EPLANET, ERC, ERDF, FP7, Horizon 2020 and Marie Skłodowska-Curie Actions, European Union; Investissements d'Avenir Labex and Idex, ANR, Région Auvergne and Fondation Partager le Savoir, France; DFG and AvH Foundation, Germany; Herakleitos, Thales and Aristeia 
programmes co-financed by EU-ESF and the Greek NSRF; BSF, GIF and Minerva, Israel; BRF, Norway; CERCA Programme Generalitat de Catalunya, Generalitat Valenciana, Spain; the Royal Society and Leverhulme Trust, United Kingdom.

The crucial computing support from all WLCG partners is acknowledged gratefully, in particular from CERN, the ATLAS Tier-1 facilities at TRIUMF (Canada), NDGF (Denmark, Norway, Sweden), CC-IN2P3 (France), KIT/GridKA (Germany), INFN-CNAF (Italy), NL-T1 (Netherlands), PIC (Spain), ASGC (Taiwan), RAL (U.K.) and BNL (U.S.A.), the Tier-2 facilities worldwide and large non-WLCG resource providers. Major contributors of computing resources are listed in ref. [56].

\section{A Results for the electron channel with $R=1.0$}

This section contains results obtained with a jet radius parameter $R=1.0$ in the electron channel. 

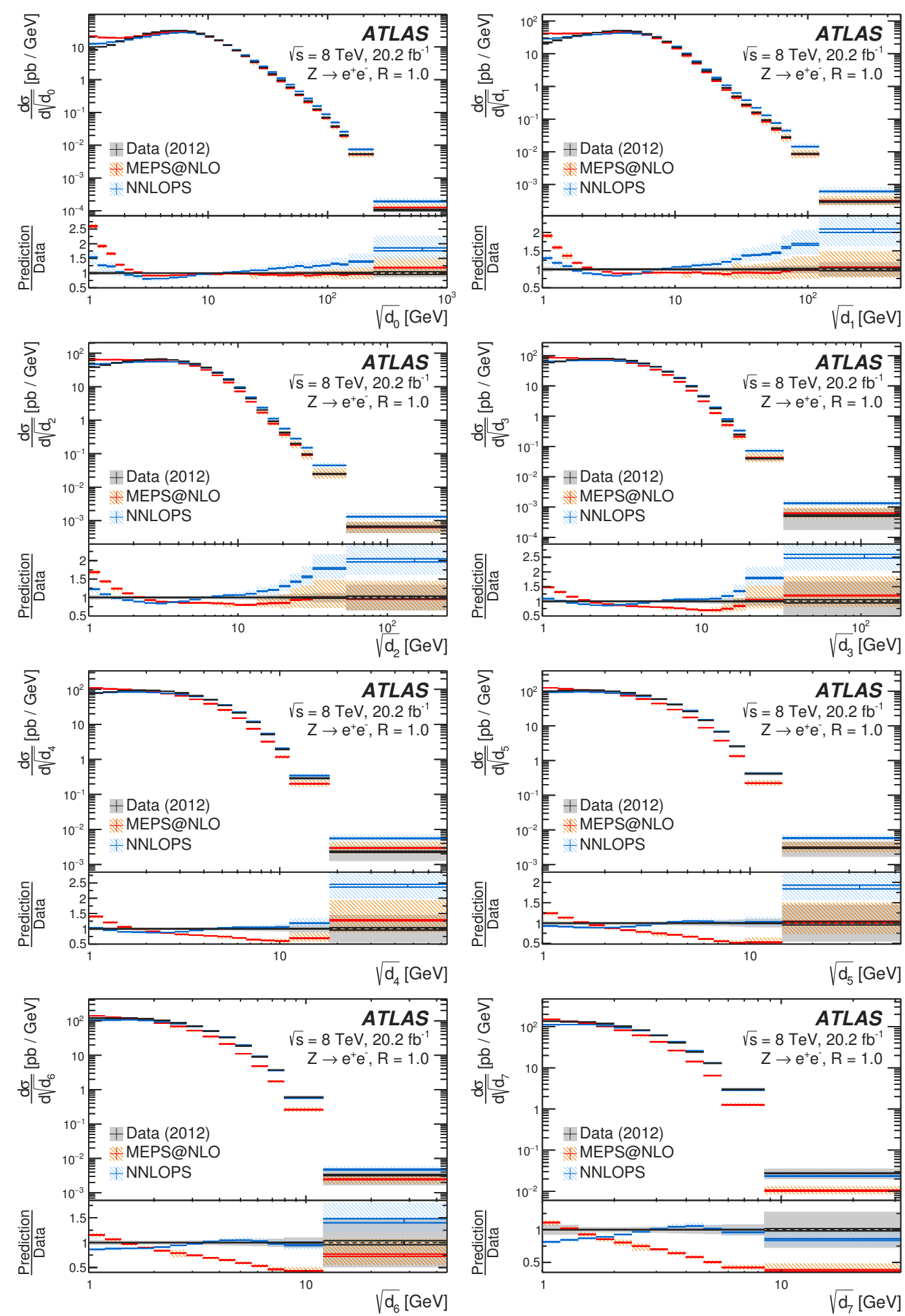

Figure 6. Charged-only distributions for the eight leading splitting scales in the electron channel using the jet-radius parameter $R=1.0$. The size of the error bars reflects the statistical uncertainty, while the combined statistical and systematic uncertainty is indicated by the grey band. Theoretical predictions from SHERPA with NLO multijet merging ("MEPS@NLO") and from PowHEG+PYTHIA 8 with NNLO matching ("NNLOPS") are displayed. 


\section{B Results for the muon channel with $R=0.4$ and $R=1.0$}

This section contains results obtained with a jet-radius parameter $R=0.4$ and $R=1.0$ in the muon channel. 

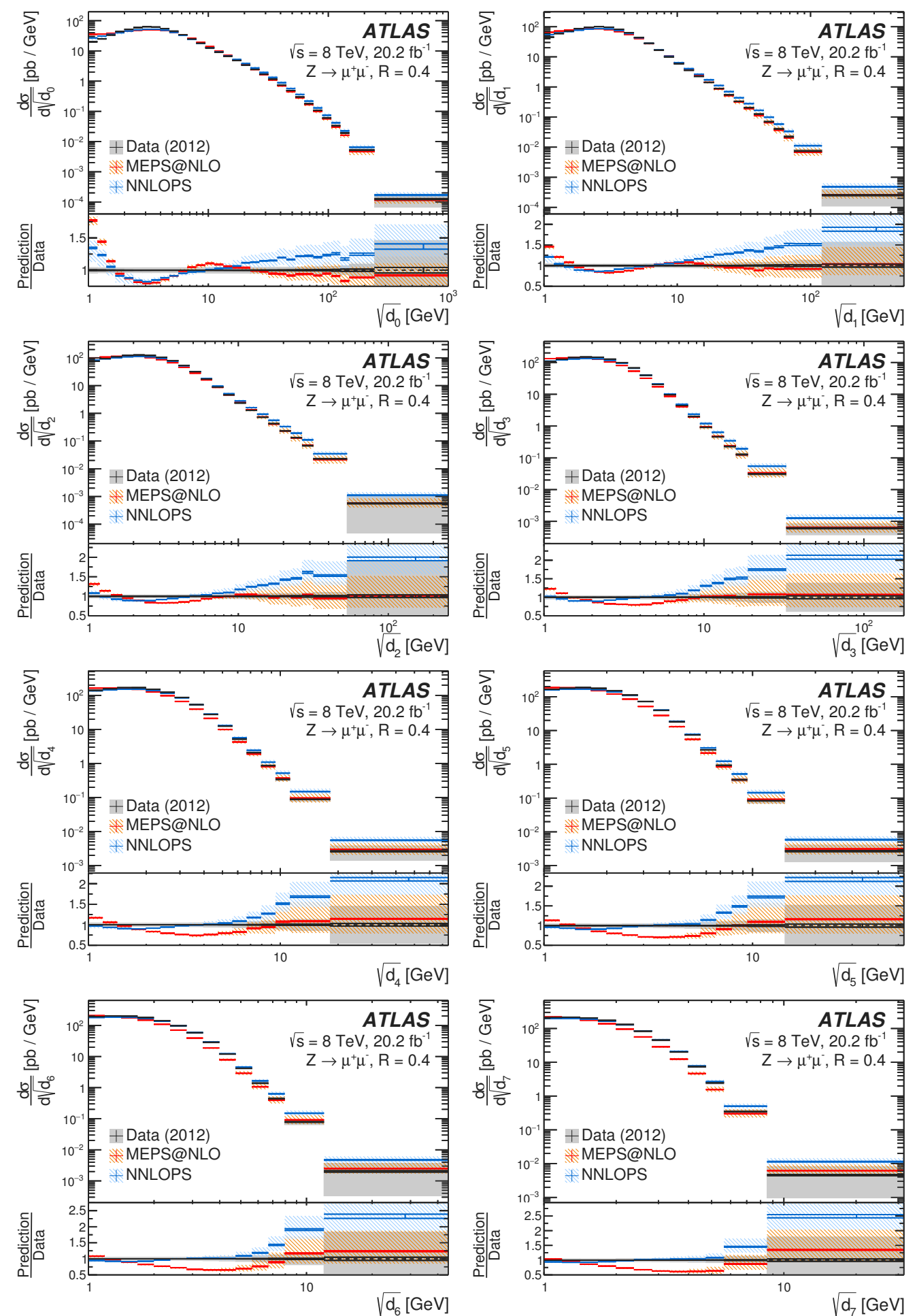

Figure 7. Charged-only distributions for the eight leading splitting scales in the muon channel using the jet-radius parameter $R=0.4$. The size of the error bars reflects the statistical uncertainty, while the combined statistical and systematic uncertainty is indicated by the grey band. Theoretical predictions from SHERPA with NLO multijet merging ("MEPS@NLO") and from PowHEG+PYTHIA 8 with NNLO matching ("NNLOPS") are displayed. 

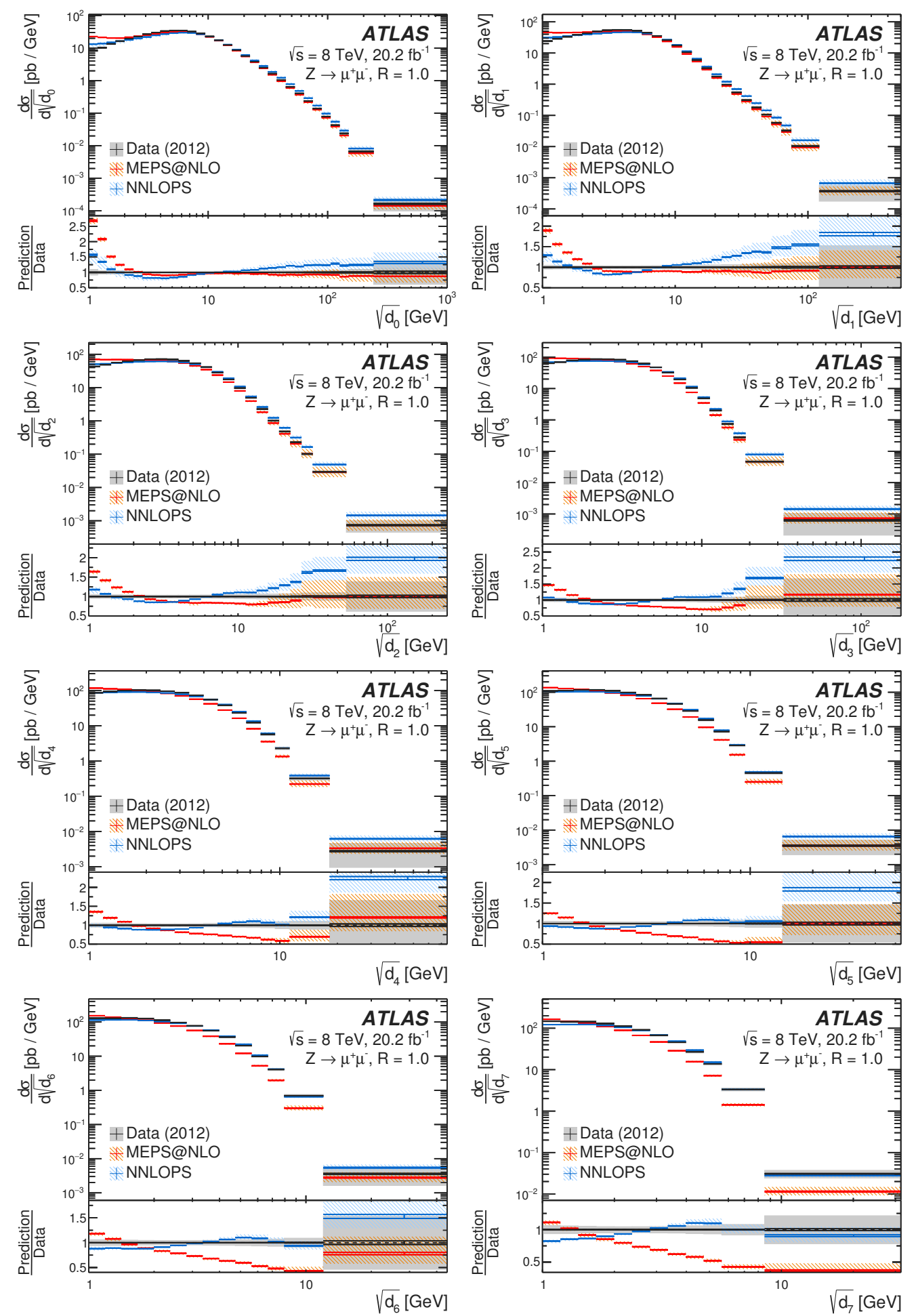

Figure 8. Charged-only distributions for the eight leading splitting scales in the muon channel using the jet-radius parameter $R=1.0$. The size of the error bars reflects the statistical uncertainty, while the combined statistical and systematic uncertainty is indicated by the grey band. Theoretical predictions from SHERPA with NLO multijet merging ("MEPS@NLO") and from PowHEG+PYTHIA 8 with NNLO matching ("NNLOPS") are displayed. 
Open Access. This article is distributed under the terms of the Creative Commons Attribution License (CC-BY 4.0), which permits any use, distribution and reproduction in any medium, provided the original author(s) and source are credited.

\section{References}

[1] H. Ita, Z. Bern, L.J. Dixon, F. Febres Cordero, D.A. Kosower and D. Maître, Precise Predictions for $Z+4$ Jets at Hadron Colliders, Phys. Rev. D 85 (2012) 031501 [arXiv: 1108.2229] [INSPIRE].

[2] Z. Bern et al., Next-to-Leading Order W +5-Jet Production at the LHC, Phys. Rev. D 88 (2013) 014025 [arXiv: 1304.1253] [INSPIRE].

[3] R. Boughezal, C. Focke, X. Liu and F. Petriello, $W$-boson production in association with a jet at next-to-next-to-leading order in perturbative QCD, Phys. Rev. Lett. 115 (2015) 062002 [arXiv: 1504.02131] [INSPIRE].

[4] S. Hoeche, F. Krauss, M. Schonherr and F. Siegert, QCD matrix elements + parton showers: The NLO case, JHEP 04 (2013) 027 [arXiv:1207.5030] [INSPIRE].

[5] R. Frederix and S. Frixione, Merging meets matching in MC@NLO, JHEP 12 (2012) 061 [arXiv: 1209.6215] [INSPIRE].

[6] L. Lönnblad and S. Prestel, Merging Multi-leg NLO Matrix Elements with Parton Showers, JHEP 03 (2013) 166 [arXiv:1211.7278] [INSPIRE].

[7] S. Höche, Y. Li and S. Prestel, Drell-Yan lepton pair production at NNLO QCD with parton showers, Phys. Rev. D 91 (2015) 074015 [arXiv: 1405.3607] [INSPIRE].

[8] A. Karlberg, E. Re and G. Zanderighi, NNLOPS accurate Drell-Yan production, JHEP 09 (2014) 134 [arXiv:1407.2940] [INSPIRE].

[9] S. Catani, Y.L. Dokshitzer, M.H. Seymour and B.R. Webber, Longitudinally invariant $K_{t}$ clustering algorithms for hadron hadron collisions, Nucl. Phys. B 406 (1993) 187 [INSPIRE].

[10] G.P. Salam, Towards Jetography, Eur. Phys. J. C 67 (2010) 637 [arXiv:0906.1833] [INSPIRE].

[11] ATLAS collaboration, Measurement of $k_{\mathrm{T}}$ splitting scales in $W \rightarrow \ell \nu$ events at $\sqrt{s}=7 \mathrm{TeV}$ withATLAS detector, Eur. Phys. J. C 73 (2013) 2432 [arXiv:1302.1415] [InSPIRE].

[12] ATLAS collaboration, The ATLAS Experiment at the CERN Large Hadron Collider, 2008 JINST 3 S08003 [INSPIRE].

[13] ATLAS collaboration, Performance of the ATLAS Trigger System in 2010, Eur. Phys. J. C 72 (2012) 1849 [arXiv:1110.1530] [inSPIRE].

[14] ATLAS collaboration, Performance of the ATLAS Inner Detector Track and Vertex Reconstruction in the High Pile-Up LHC Environment, ATLAS-CONF-2012-042 (2012).

[15] ATLAS collaboration, Electron efficiency measurements with the ATLAS detector using 2012 LHC proton-proton collision data, Eur. Phys. J. C 77 (2017) 195 [arXiv:1612.01456] [INSPIRE].

[16] ATLAS collaboration, Measurement of the muon reconstruction performance of the ATLAS detector using 2011 and 2012 LHC proton-proton collision data, Eur. Phys. J. C 74 (2014) 3130 [arXiv: 1407.3935 ] [INSPIRE].

[17] P. Nason, A new method for combining NLO QCD with shower Monte Carlo algorithms, JHEP 11 (2004) 040 [hep-ph/0409146] [INSPIRE]. 
[18] S. Frixione, P. Nason and C. Oleari, Matching NLO QCD computations with Parton Shower simulations: the POWHEG method, JHEP 11 (2007) 070 [arXiv:0709.2092] [INSPIRE].

[19] S. Alioli, P. Nason, C. Oleari and E. Re, A general framework for implementing NLO calculations in shower Monte Carlo programs: the POWHEG BOX, JHEP 06 (2010) 043 [arXiv: 1002.2581] [INSPIRE].

[20] H.-L. Lai et al., New parton distributions for collider physics, Phys. Rev. D 82 (2010) 074024 [arXiv: 1007.2241] [INSPIRE].

[21] P. Golonka and Z. Was, PHOTOS Monte Carlo: A precision tool for QED corrections in Z and $W$ decays, Eur. Phys. J. C 45 (2006) 97 [hep-ph/0506026] [INSPIRE].

[22] S. Hoche, F. Krauss, M. Schonherr and F. Siegert, NLO matrix elements and truncated showers, JHEP 08 (2011) 123 [arXiv: 1009.1127] [INSPIRE].

[23] C. Anastasiou, L.J. Dixon, K. Melnikov and F. Petriello, High precision QCD at hadron colliders: Electroweak gauge boson rapidity distributions at NNLO, Phys. Rev. D 69 (2004) 094008 [hep-ph/0312266] [INSPIRE].

[24] T. Sjöstrand, S. Mrenna and P.Z. Skands, PYTHIA 6.4 Physics and Manual, JHEP 05 (2006) 026 [hep-ph/0603175] [INSPIRE].

[25] J. Pumplin, D.R. Stump, J. Huston, H.L. Lai, P.M. Nadolsky and W.K. Tung, New generation of parton distributions with uncertainties from global QCD analysis, JHEP 07 (2002) 012 [hep-ph/0201195] [INSPIRE].

[26] P.Z. Skands, Tuning Monte Carlo Generators: The Perugia Tunes, Phys. Rev. D 82 (2010) 074018 [arXiv: 1005.3457] [INSPIRE].

[27] M. Czakon, P. Fiedler and A. Mitov, Total Top-Quark Pair-Production Cross section at Hadron Colliders Through $O\left(\alpha \frac{4}{S}\right)$, Phys. Rev. Lett. 110 (2013) 252004 [arXiv:1303.6254] [INSPIRE].

[28] N. Kidonakis, Two-loop soft anomalous dimensions for single top quark associated production with $a W^{-}$or $H^{-}$, Phys. Rev. D 82 (2010) 054018 [arXiv:1005.4451] [InSPIRE].

[29] N. Kidonakis, NNLL resummation for s-channel single top quark production, Phys. Rev. D 81 (2010) 054028 [arXiv: 1001.5034] [INSPIRE].

[30] N. Kidonakis, Next-to-next-to-leading-order collinear and soft gluon corrections for t-channel single top quark production, Phys. Rev. D 83 (2011) 091503 [arXiv:1103.2792] [INSPIRE].

[31] GEANT4 collaboration, S. Agostinelli et al., GEANT4: A simulation toolkit, Nucl. Instrum. Meth. A 506 (2003) 250 [inSPIRE].

[32] J. Allison et al., Geant4 developments and applications, IEEE Trans. Nucl. Sci. 53 (2006) 270 .

[33] ATLAS collaboration, The ATLAS Simulation Infrastructure, Eur. Phys. J. C 70 (2010) 823 [arXiv: 1005.4568] [INSPIRE].

[34] T. Sjöstrand, S. Mrenna and P.Z. Skands, A Brief Introduction to PYTHIA 8.1, Comput. Phys. Commun. 178 (2008) 852 [arXiv:0710.3820] [INSPIRE].

[35] ATLAS collaboration, Further ATLAS tunes of PYTHIA6 and PYTHIA 8, ATL-PHYS-PUB-2011-014 (2011).

[36] A.D. Martin, W.J. Stirling, R.S. Thorne and G. Watt, Parton distributions for the LHC, Eur. Phys. J. C 63 (2009) 189 [arXiv:0901.0002] [INSPIRE]. 
[37] T. Gleisberg et al., Event generation with SHERPA 1.1, JHEP 02 (2009) 007 [arXiv:0811.4622] [INSPIRE].

[38] T. Gleisberg and S. Hoeche, Comix, a new matrix element generator, JHEP 12 (2008) 039 [arXiv:0808.3674] [INSPIRE].

[39] F. Cascioli, P. Maierhofer and S. Pozzorini, Scattering Amplitudes with Open Loops, Phys. Rev. Lett. 108 (2012) 111601 [arXiv:1111.5206] [INSPIRE].

[40] S. Schumann and F. Krauss, A parton shower algorithm based on Catani-Seymour dipole factorisation, JHEP 03 (2008) 038 [arXiv:0709.1027] [INSPIRE].

[41] S. Catani, F. Krauss, R. Kuhn and B.R. Webber, QCD matrix elements + parton showers, JHEP 11 (2001) 063 [hep-ph/0109231] [INSPIRE].

[42] S. Hoeche, F. Krauss, S. Schumann and F. Siegert, QCD matrix elements and truncated showers, JHEP 05 (2009) 053 [arXiv:0903.1219] [INSPIRE].

[43] NNPDF collaboration, R.D. Ball et al., Parton distributions for the LHC Run II, JHEP 04 (2015) 040 [arXiv: 1410.8849] [INSPIRE].

[44] S. Catani, L. Cieri, G. Ferrera, D. de Florian and M. Grazzini, Vector boson production at hadron colliders: a fully exclusive QCD calculation at NNLO, Phys. Rev. Lett. 103 (2009) 082001 [arXiv: 0903.2120] [INSPIRE].

[45] S. Catani and M. Grazzini, An NNLO subtraction formalism in hadron collisions and its application to Higgs boson production at the LHC, Phys. Rev. Lett. 98 (2007) 222002 [hep-ph/0703012] [INSPIRE].

[46] K. Hamilton, P. Nason, C. Oleari and G. Zanderighi, Merging $H / W / Z+0$ and 1 jet at $N L O$ with no merging scale: a path to parton shower + NNLO matching, JHEP 05 (2013) 082 [arXiv:1212.4504] [INSPIRE].

[47] S. Alioli, P. Nason, C. Oleari and E. Re, Vector boson plus one jet production in POWHEG, JHEP 01 (2011) 095 [arXiv: 1009.5594] [INSPIRE].

[48] J.R. Andersen et al., Les Houches 2015: Physics at TeV Colliders Standard Model Working Group Report, arXiv:1605.04692 [INSPIRE].

[49] P. Skands, S. Carrazza and J. Rojo, Tuning PYTHIA 8.1: the Monash 2013 Tune, Eur. Phys. J. C 74 (2014) 3024 [arXiv: 1404.5630] [INSPIRE].

[50] G. D'Agostini, A multidimensional unfolding method based on Bayes' theorem, Nucl. Instrum. Meth. A 362 (1995) 487 [INSPIRE].

[51] T. Adye, Unfolding algorithms and tests using RooUnfold, arXiv:1105.1160 [INSPIRE].

[52] ATLAS collaboration, Luminosity determination in pp collisions at $\sqrt{s}=8 \mathrm{TeV}$ using the ATLAS detector at the LHC, Eur. Phys. J. C 76 (2016) 653 [arXiv:1608.03953] [InSPIRE].

[53] A. Buckley et al., LHAPDF6: parton density access in the LHC precision era, Eur. Phys. J. C 75 (2015) 132 [arXiv:1412.7420] [inSPIRE].

[54] A. Buckley and M. Whalley, HepData reloaded: Reinventing the HEP data archive, PoS (ACAT2010) 067 [arXiv: 1006.0517] [INSPIRE].

[55] A. Buckley et al., Rivet user manual, Comput. Phys. Commun. 184 (2013) 2803 [arXiv: 1003.0694] [INSPIRE].

[56] ATLAS collaboration, ATLAS Computing Acknowledgements 2016-201\%, ATL-GEN-PUB-2016-002 (2016). 


\section{The ATLAS collaboration}

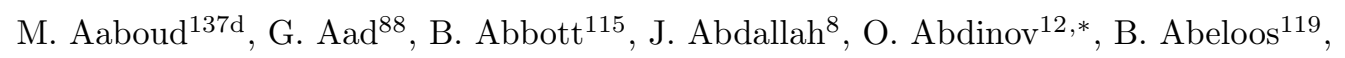
S.H. Abidi ${ }^{161}$, O.S. AbouZeid ${ }^{139}$, N.L. Abraham ${ }^{151}$, H. Abramowicz ${ }^{155}$, H. Abreu ${ }^{154}$, R. Abreu ${ }^{118}$, Y. Abulaiti ${ }^{148 a, 148 b}$, B.S. Acharya ${ }^{167 a, 167 b, a}$, S. Adachi ${ }^{157}$, L. Adamczyk ${ }^{41 a}$, J. Adelman ${ }^{110}$, M. Adersberger ${ }^{102}$, T. Adye ${ }^{133}$, A.A. Affolder ${ }^{139}$, T. Agatonovic-Jovin ${ }^{14}$, C. Agheorghiesei ${ }^{28 c}$, J.A. Aguilar-Saavedra ${ }^{128 a, 128 f}$, S.P. Ahlen ${ }^{24}$, F. Ahmadov ${ }^{68, b}$, G. Aielli135a,135b, S. Akatsuka ${ }^{71}$, H. Akerstedt ${ }^{148 a, 148 b}$, T.P.A. Åkesson ${ }^{84}$, A.V. Akimov ${ }^{98}$, G.L. Alberghi ${ }^{22 a, 22 b}$, J. Albert ${ }^{172}$, P. Albicocco ${ }^{50}$, M.J. Alconada Verzini ${ }^{74}$, M. Aleksa ${ }^{32}$, I.N. Aleksandrov ${ }^{68}$, C. Alexa ${ }^{28 b}$, G. Alexander ${ }^{155}$, T. Alexopoulos ${ }^{10}$, M. Alhroob ${ }^{115}$, B. Ali ${ }^{130}$, M. Aliev ${ }^{76 a, 76 b}$, G. Alimonti ${ }^{94 a}$, J. Alison ${ }^{33}$, S.P. Alkire ${ }^{38}$, B.M.M. Allbrooke ${ }^{151}$, B.W. Allen ${ }^{118}$, P.P. Allport ${ }^{19}$, A. Aloisio ${ }^{106 a, 106 b}$, A. Alonso ${ }^{39}$, F. Alonso ${ }^{74}$, C. Alpigiani ${ }^{140}$, A.A. Alshehri ${ }^{56}$, M. Alstaty ${ }^{88}$, B. Alvarez Gonzalez ${ }^{32}$, D. Álvarez Piqueras ${ }^{170}$, M.G. Alviggi ${ }^{106 a, 106 b}$, B.T. Amadio ${ }^{16}$, Y. Amaral Coutinho ${ }^{26 a}$, C. Amelung ${ }^{25}$, D. Amidei ${ }^{92}$, S.P. Amor Dos Santos ${ }^{128 a, 128 c}$, A. Amorim ${ }^{128 a, 128 b}$, S. Amoroso ${ }^{32}$, G. Amundsen ${ }^{25}$, C. Anastopoulos ${ }^{141}$, L.S. Ancu ${ }^{52}$, N. Andari ${ }^{19}$, T. Andeen ${ }^{11}$, C.F. Anders ${ }^{60 b}$, J.K. Anders ${ }^{77}$, K.J. Anderson ${ }^{33}$, A. Andreazza ${ }^{94 a, 94 b}$, V. Andrei60a , S. Angelidakis ${ }^{9}$, I. Angelozzi ${ }^{109}$, A. Angerami ${ }^{38}$, A.V. Anisenkov ${ }^{111, c}$, N. Anjos ${ }^{13}$, A. Annovi ${ }^{126 a, 126 b}$, C. Antel ${ }^{60 a}$, M. Antonelli ${ }^{50}$, A. Antonov ${ }^{100, *}$, D.J. Antrim ${ }^{166}$, F. Anulli ${ }^{134 a}$, M. Aoki ${ }^{69}$, L. Aperio Bella ${ }^{32}$, G. Arabidze ${ }^{93}$, Y. Arai ${ }^{69}$, J.P. Araque ${ }^{128 a}$, V. Araujo Ferraz ${ }^{26 a}$, A.T.H. Arce ${ }^{48}$, R.E. Ardell ${ }^{80}$, F.A. Arduh ${ }^{74}$, J-F. Arguin 97 , S. Argyropoulos ${ }^{66}$, M. Arik ${ }^{20 a}$, A.J. Armbruster ${ }^{145}$,

L.J. Armitage ${ }^{79}$, O. Arnaez ${ }^{161}$, H. Arnold ${ }^{51}$, M. Arratia ${ }^{30}$, O. Arslan ${ }^{23}$, A. Artamonov 99 , G. Artoni ${ }^{122}$, S. Artz ${ }^{86}$, S. Asai ${ }^{157}$, N. Asbah ${ }^{45}$, A. Ashkenazi ${ }^{155}$, L. Asquith ${ }^{151}$, K. Assamagan ${ }^{27}$, R. Astalos ${ }^{146 a}$, M. Atkinson ${ }^{169}$, N.B. Atlay ${ }^{143}$, K. Augsten ${ }^{130}$, G. Avolio ${ }^{32}$, B. Axen ${ }^{16}$, M.K. Ayoub ${ }^{119}$, G. Azuelos ${ }^{97, d}$, A.E. Baas ${ }^{60 a}$, M.J. Baca ${ }^{19}$, H. Bachacou ${ }^{138}$, K. Bachas ${ }^{76 a, 76 b}$, M. Backes ${ }^{122}$, M. Backhaus ${ }^{32}$, P. Bagnaia ${ }^{134 a, 134 b}$, H. Bahrasemani ${ }^{144}$, J.T. Baines ${ }^{133}$, M. Bajic ${ }^{39}$, O.K. Baker ${ }^{179}$, E.M. Baldin ${ }^{111, c}$, P. Balek ${ }^{175}$, F. Balli ${ }^{138}$, W.K. Balunas ${ }^{124}$, E. Banas ${ }^{42}$, Sw. Banerjee ${ }^{176, e}$, A.A.E. Bannoura ${ }^{178}$, L. Barak ${ }^{32}$, E.L. Barberio ${ }^{91}$, D. Barberis ${ }^{53 a, 53 b}$, M. Barbero ${ }^{88}$, T. Barillari ${ }^{103}$, M-S Barisits ${ }^{32}$, T. Barklow ${ }^{145}$, N. Barlow ${ }^{30}$, S.L. Barnes ${ }^{36 c}$, B.M. Barnett ${ }^{133}$, R.M. Barnett ${ }^{16}$, Z. Barnovska-Blenessy ${ }^{36 a}$, A. Baroncelli ${ }^{136 a}$, G. Barone ${ }^{25}$, A.J. Barr ${ }^{122}$, L. Barranco Navarro ${ }^{170}$, F. Barreiro ${ }^{85}$, J. Barreiro Guimarães da Costa ${ }^{35 a}$, R. Bartoldus ${ }^{145}$, A.E. Barton ${ }^{75}$, P. Bartos ${ }^{146 a}$, A. Basalaev ${ }^{125}$, A. Bassalat ${ }^{119, f}$, R.L. Bates ${ }^{56}$, S.J. Batista ${ }^{161}$, J.R. Batley ${ }^{30}$, M. Battaglia ${ }^{139}$, M. Bauce ${ }^{134 a, 134 b}$, F. Bauer ${ }^{138}$, H.S. Bawa ${ }^{145, g}$, J.B. Beacham ${ }^{113}$, M.D. Beattie ${ }^{75}$, T. Beau ${ }^{83}$, P.H. Beauchemin ${ }^{165}$, P. Bechtle ${ }^{23}$, H.P. Beck ${ }^{18, h}$, K. Becker ${ }^{122}$, M. Becker ${ }^{86}$, M. Beckingham ${ }^{173}$, C. Becot ${ }^{112}$, A.J. Beddall ${ }^{20 e}$, A. Beddall ${ }^{20 b}$, V.A. Bednyakov ${ }^{68}$, M. Bedognetti ${ }^{109}$, C.P. Bee ${ }^{150}$, T.A. Beermann ${ }^{32}$, M. Begalli ${ }^{26 a}$, M. Begel ${ }^{27}$, J.K. Behr ${ }^{45}$, A.S. Bell ${ }^{81}$, G. Bella ${ }^{155}$, L. Bellagamba ${ }^{22 a}$, A. Bellerive ${ }^{31}$, M. Bellomo ${ }^{154}$, K. Belotskiy ${ }^{100}$, O. Beltramello ${ }^{32}$, N.L. Belyaev ${ }^{100}$, O. Benary ${ }^{155, *}$, D. Benchekroun ${ }^{137 a}$, M. Bender ${ }^{102}$, K. Bendtz ${ }^{148 a, 148 b}$, N. Benekos ${ }^{10}$, Y. Benhammou ${ }^{155}$, E. Benhar Noccioli ${ }^{179}$, J. Benitez ${ }^{66}$, D.P. Benjamin ${ }^{48}$, M. Benoit ${ }^{52}$, J.R. Bensinger ${ }^{25}$, S. Bentvelsen ${ }^{109}$, L. Beresford ${ }^{122}$, M. Beretta ${ }^{50}$, D. Berge ${ }^{109}$, E. Bergeaas Kuutmann ${ }^{168}$, N. Berger ${ }^{5}$, J. Beringer ${ }^{16}$, S. Berlendis ${ }^{58}$, N.R. Bernard ${ }^{89}$, G. Bernardi ${ }^{83}$, C. Bernius ${ }^{145}$, F.U. Bernlochner ${ }^{23}$, T. Berry ${ }^{80}$, P. Berta ${ }^{131}$, C. Bertella ${ }^{35 a}$, G. Bertoli ${ }^{148 a, 148 b}$, F. Bertolucci ${ }^{126 a, 126 b}$, I.A. Bertram ${ }^{75}$, C. Bertsche ${ }^{45}$, D. Bertsche ${ }^{115}$, G.J. Besjes ${ }^{39}$, O. Bessidskaia Bylund ${ }^{148 a, 148 b}$, M. Bessner ${ }^{45}$, N. Besson ${ }^{138}$, C. Betancourt ${ }^{51}$, A. Bethani ${ }^{87}$, S. Bethke ${ }^{103}$, A.J. Bevan ${ }^{79}$, J. Beyer ${ }^{103}$, R.M. Bianchi ${ }^{127}$, O. Biebel ${ }^{102}$, D. Biedermann ${ }^{17}$, R. Bielski ${ }^{87}$, N.V. Biesuz ${ }^{126 a, 126 b}$, M. Biglietti ${ }^{136 a}$,

J. Bilbao De Mendizabal ${ }^{52}$, T.R.V. Billoud ${ }^{97}$, H. Bilokon ${ }^{50}$, M. Bindi ${ }^{57}$, A. Bingul ${ }^{20 b}$, C. Bini ${ }^{134 a, 134 b}$, S. Biondi ${ }^{22 a, 22 b}$, T. Bisanz ${ }^{57}$, C. Bittrich ${ }^{47}$, D.M. Bjergaard ${ }^{48}$, C.W. Black ${ }^{152}$, J.E. Black ${ }^{145}$, K.M. Black ${ }^{24}$, R.E. Blair ${ }^{6}$, T. Blazek ${ }^{146 a}$, I. Bloch ${ }^{45}$, C. Blocker ${ }^{25}$, A. Blue ${ }^{56}$, 
W. Blum ${ }^{86, *}$, U. Blumenschein ${ }^{79}$, S. Blunier ${ }^{34 a}$, G.J. Bobbink ${ }^{109}$, V.S. Bobrovnikov ${ }^{111, c}$, S.S. Bocchetta ${ }^{84}$, A. Bocci ${ }^{48}$, C. Bock ${ }^{102}$, M. Boehler ${ }^{51}$, D. Boerner ${ }^{178}$, D. Bogavac ${ }^{102}$, A.G. Bogdanchikov ${ }^{111}$, C. Bohm ${ }^{148 a}$, V. Boisvert ${ }^{80}$, P. Bokan ${ }^{168, i}$, T. Bold ${ }^{41 a}$, A.S. Boldyrev ${ }^{101}$, A.E. Bolz ${ }^{60 b}$, M. Bomben ${ }^{83}$, M. Bona ${ }^{79}$, M. Boonekamp ${ }^{138}$, A. Borisov ${ }^{132}$, G. Borissov ${ }^{75}$, J. Bortfeldt ${ }^{32}$, D. Bortoletto ${ }^{122}$, V. Bortolotto ${ }^{62 \mathrm{a}, 62 \mathrm{~b}, 62 \mathrm{c}}, \mathrm{K}^{2}$ Bos $^{109}$, D. Boscherini ${ }^{22 \mathrm{a}}$, M. Bosman ${ }^{13}$, J.D. Bossio Sola ${ }^{29}$, J. Boudreau ${ }^{127}$, J. Bouffard ${ }^{2}$, E.V. Bouhova-Thacker ${ }^{75}$, D. Boumediene ${ }^{37}$, C. Bourdarios ${ }^{119}$, S.K. Boutle ${ }^{56}$, A. Boveia ${ }^{113}$, J. Boyd ${ }^{32}$, I.R. Boyko ${ }^{68}$,

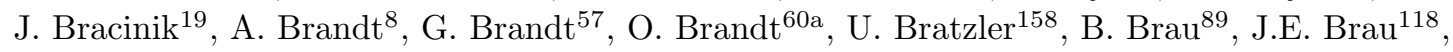
W.D. Breaden Madden ${ }^{56}$, K. Brendlinger ${ }^{45}$, A.J. Brennan ${ }^{91}$, L. Brenner ${ }^{109}$, R. Brenner ${ }^{168}$, S. Bressler ${ }^{175}$, D.L. Briglin ${ }^{19}$, T.M. Bristow ${ }^{49}$, D. Britton ${ }^{56}$, D. Britzger ${ }^{45}$, F.M. Brochu ${ }^{30}$, I. Brock $^{23}$, R. Brock ${ }^{93}$, G. Brooijmans ${ }^{38}$, T. Brooks ${ }^{80}$, W.K. Brooks ${ }^{34 b}$, J. Brosamer ${ }^{16}$, E. Brost ${ }^{110}$, J.H Broughton ${ }^{19}$, P.A. Bruckman de Renstrom ${ }^{42}$, D. Bruncko ${ }^{146 b}$, A. Bruni ${ }^{22 a}$, G. Bruni ${ }^{22 a}$, L.S. Bruni ${ }^{109}$, BH Brunt ${ }^{30}$, M. Bruschi ${ }^{22 a}$, N. Bruscino ${ }^{23}$, P. Bryant $^{33}$, L. Bryngemark ${ }^{45}$, T. Buanes ${ }^{15}$, Q. Buat ${ }^{144}$, P. Buchholz ${ }^{143}$, A.G. Buckley ${ }^{56}$, I.A. Budagov ${ }^{68}$, F. Buehrer ${ }^{51}$, M.K. Bugge ${ }^{121}$, O. Bulekov ${ }^{100}$, D. Bullock ${ }^{8}$, T.J. Burch ${ }^{110}$, H. Burckhart ${ }^{32}$, S. Burdin ${ }^{77}$, C.D. Burgard ${ }^{51}$, A.M. Burger ${ }^{5}$, B. Burghgrave ${ }^{110}$, K. Burka ${ }^{42}$, S. Burke ${ }^{133}$, I. Burmeister ${ }^{46}$, J.T.P. Burr ${ }^{122}$, E. Busato ${ }^{37}$, D. Büscher ${ }^{51}$, V. Büscher ${ }^{86}$, P. Bussey ${ }^{56}$, J.M. Butler ${ }^{24}$, C.M. Buttar ${ }^{56}$, J.M. Butterworth ${ }^{81}$, P. Butti ${ }^{32}$, W. Buttinger ${ }^{27}$, A. Buzatu ${ }^{35 c}$, A.R. Buzykaev ${ }^{111, c}$, S. Cabrera Urbán ${ }^{170}$, D. Caforio ${ }^{130}$, V.M. Cairo ${ }^{40 a, 40 b}$, O. Cakir ${ }^{4 a}$, N. Calace ${ }^{52}$, P. Calafiura ${ }^{16}$, A. Calandri ${ }^{88}$, G. Calderini ${ }^{83}$, P. Calfayan ${ }^{64}$, G. Callea ${ }^{40 a, 40 b}$, L.P. Caloba ${ }^{26 a}$, S. Calvente Lopez ${ }^{85}$, D. Calvet ${ }^{37}$, S. Calvet ${ }^{37}$, T.P. Calvet ${ }^{88}$, R. Camacho Toro ${ }^{33}$, S. Camarda ${ }^{32}$, P. Camarri ${ }^{135 a, 135 b}$, D. Cameron ${ }^{121}$, R. Caminal Armadans ${ }^{169}$, C. Camincher ${ }^{58}$, S. Campana ${ }^{32}$, M. Campanelli ${ }^{81}$, A. Camplani ${ }^{94 a, 94 b}$, A. Campoverde ${ }^{143}$, V. Canale ${ }^{106 a, 106 b}$, M. Cano Bret ${ }^{36 c}$, J. Cantero ${ }^{116}$, T. Cao ${ }^{155}$, M.D.M. Capeans Garrido ${ }^{32}$, I. Caprini28b, M. Caprini ${ }^{28 b}$, M. Capua ${ }^{40 a, 40 b}$, R.M. Carbone ${ }^{38}$, R. Cardarelli ${ }^{135 a}$, F. Cardillo ${ }^{51}$, I. Carli ${ }^{131}$, T. Carli ${ }^{32}$, G. Carlino ${ }^{106 a}$, B.T. Carlson ${ }^{127}$, L. Carminati94a,94b, R.M.D. Carney ${ }^{148 a, 148 b}$, S. Caron ${ }^{108}$, E. Carquin ${ }^{34 b}$, S. Carrá94a,94b , G.D. Carrillo-Montoya ${ }^{32}$, J. Carvalho ${ }^{128 a, 128 c}$, D. Casadei ${ }^{19}$, M.P. Casado ${ }^{13, j}$, M. Casolino ${ }^{13}$, D.W. Casper ${ }^{166}$, R. Castelijn ${ }^{109}$, V. Castillo Gimenez ${ }^{170}$, N.F. Castro ${ }^{128 a, k}$, A. Catinaccio ${ }^{32}$, J.R. Catmore ${ }^{121}$, A. Cattai ${ }^{32}$, J. Caudron ${ }^{23}$, V. Cavaliere ${ }^{169}$, E. Cavallaro ${ }^{13}$, D. Cavalli ${ }^{94 a}$, M. Cavalli-Sforza ${ }^{13}$, V. Cavasinni ${ }^{126 a, 126 b}$, E. Celebi ${ }^{20 a}$, F. Ceradini ${ }^{136 a, 136 b}$, L. Cerda Alberich ${ }^{170}$, A.S. Cerqueira ${ }^{26 b}$, A. Cerri ${ }^{151}$, L. Cerrito ${ }^{135 a, 135 b}$, F. Cerutti ${ }^{16}$, A. Cervelli ${ }^{18}$, S.A. Cetin $^{20 d}$, A. Chafaq ${ }^{137 a}$, D. Chakraborty ${ }^{110}$, S.K. Chan ${ }^{59}$, W.S. Chan ${ }^{109}$, Y.L. Chan ${ }^{62 a}$, P. Chang ${ }^{169}$, J.D. Chapman ${ }^{30}$, D.G. Charlton ${ }^{19}$, C.C. Chau ${ }^{161}$, C.A. Chavez Barajas ${ }^{151}$, S. Che ${ }^{113}$, S. Cheatham ${ }^{167 a, 167 c}$, A. Chegwidden ${ }^{93}$, S. Chekanov ${ }^{6}$, S.V. Chekulaev ${ }^{163 a}$, G.A. Chelkov ${ }^{68, l}$, M.A. Chelstowska ${ }^{32}$, C. Chen ${ }^{67}$, H. Chen ${ }^{27}$, S. Chen ${ }^{35 b}$, S. Chen ${ }^{157}$, X. Chen ${ }^{35 c, m}$, Y. Chen ${ }^{70}$, H.C. Cheng ${ }^{92}$, H.J. Cheng ${ }^{35 a}$, A. Cheplakov ${ }^{68}$, E. Cheremushkina ${ }^{132}$, R. Cherkaoui El Moursli ${ }^{137 e}$,

V. Chernyatin ${ }^{27, *}$, E. Cheu ${ }^{7}$, L. Chevalier ${ }^{138}$, V. Chiarella ${ }^{50}$, G. Chiarelli ${ }^{126 a, 126 b}$, G. Chiodini ${ }^{76 a}$, A.S. Chisholm ${ }^{32}$, A. Chitan ${ }^{28 b}$, Y.H. Chiu ${ }^{172}$, M.V. Chizhov ${ }^{68}$, K. Choi ${ }^{64}$, A.R. Chomont ${ }^{37}$, S. Chouridou ${ }^{156}$, V. Christodoulou ${ }^{81}$, D. Chromek-Burckhart ${ }^{32}$, M.C. Chu ${ }^{62 a}$, J. Chudoba ${ }^{129}$, A.J. Chuinard ${ }^{90}$, J.J. Chwastowski ${ }^{42}$, L. Chytka ${ }^{117}$, A.K. Ciftci ${ }^{4 a}$, D. Cinca ${ }^{46}$, V. Cindro ${ }^{78}$, I.A. Cioara ${ }^{23}$, C. Ciocca ${ }^{22 a, 22 b}$, A. Ciocio ${ }^{16}$, F. Cirotto ${ }^{106 a, 106 b}$, Z.H. Citron ${ }^{175}$, M. Citterio ${ }^{94 a}$, M. Ciubancan ${ }^{28 b}$, A. Clark ${ }^{52}$, B.L. Clark ${ }^{59}$, M.R. Clark ${ }^{38}$, P.J. Clark ${ }^{49}$, R.N. Clarke ${ }^{16}$, C. Clement ${ }^{148 a, 148 b}$, Y. Coadou ${ }^{88}$, M. Cobal ${ }^{167 a, 167 c}$, A. Coccaro ${ }^{52}$, J. Cochran ${ }^{67}$, L. Colasurdo $^{108}$, B. Cole ${ }^{38}$, A.P. Colijn ${ }^{109}$, J. Collot ${ }^{58}$, T. Colombo ${ }^{166}$, P. Conde Muiño ${ }^{128 a, 128 b}$, E. Coniavitis ${ }^{51}$, S.H. Connell ${ }^{147 b}$, I.A. Connelly ${ }^{87}$, S. Constantinescu ${ }^{28 b}$, G. Conti $^{32}$, F. Conventi ${ }^{106 a, n}$, M. Cooke ${ }^{16}$, A.M. Cooper-Sarkar ${ }^{122}$, F. Cormier ${ }^{171}$, K.J.R. Cormier ${ }^{161}$, M. Corradi ${ }^{134 a, 134 b}$, F. Corriveau ${ }^{90, o}$, A. Cortes-Gonzalez ${ }^{32}$, G. Cortiana ${ }^{103}$, G. Costa ${ }^{94 a}$, M.J. Costa ${ }^{170}$, 


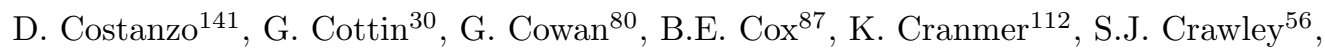
R.A. Creager ${ }^{124}$, G. Cree $^{31}$, S. Crépé-Renaudin ${ }^{58}$, F. Crescioli ${ }^{83}$, W.A. Cribbs ${ }^{148 a, 148 b}$, M. Cristinziani ${ }^{23}$, V. Croft ${ }^{108}$, G. Crosetti ${ }^{40 a, 40 b}$, A. Cueto ${ }^{85}$, T. Cuhadar Donszelmann ${ }^{141}$, A.R. Cukierman ${ }^{145}$, J. Cummings ${ }^{179}$, M. Curatolo ${ }^{50}$, J. Cúth ${ }^{86}$, H. Czirr ${ }^{143}$, P. Czodrowski ${ }^{32}$, G. D'amen ${ }^{22 a, 22 b}$, S. D'Auria ${ }^{56}$, L. D'eramo ${ }^{83}$, M. D'Onofrio ${ }^{77}$,

M.J. Da Cunha Sargedas De Sousa ${ }^{128 a}, 128 b$, C. Da Via ${ }^{87}$, W. Dabrowski ${ }^{41 a}$, T. Dado ${ }^{146 a}$, T. Dai ${ }^{92}$, O. Dale ${ }^{15}$, F. Dallaire ${ }^{97}$, C. Dallapiccola ${ }^{89}$, M. Dam ${ }^{39}$, J.R. Dandoy ${ }^{124}$, M.F. Daneri ${ }^{29}$, N.P. Dang ${ }^{176}$, A.C. Daniells ${ }^{19}$, N.S. Dann ${ }^{87}$, M. Danninger ${ }^{171}$, M. Dano Hoffmann ${ }^{138}$, V. Dao ${ }^{150}$, G. Darbo ${ }^{53 a}$, S. Darmora ${ }^{8}$, J. Dassoulas ${ }^{3}$, A. Dattagupta ${ }^{118}$, T. Daubney ${ }^{45}$, W. Davey ${ }^{23}$, C. David ${ }^{45}$, T. Davidek ${ }^{131}$, M. Davies ${ }^{155}$, D.R. Davis ${ }^{48}$, P. Davison ${ }^{81}$, E. Dawe ${ }^{91}$, I. Dawson ${ }^{141}$, K. De ${ }^{8}$, R. de Asmundis ${ }^{106 a}$, A. De Benedetti ${ }^{115}$, S. De Castro ${ }^{22 a, 22 b}$, S. De Cecco ${ }^{83}$,

N. De Groot ${ }^{108}$, P. de Jong ${ }^{109}$, H. De la Torre ${ }^{93}$, F. De Lorenzi ${ }^{67}$, A. De Maria ${ }^{57}$, D. De Pedis ${ }^{134 a}$, A. De Salvo ${ }^{134 a}$, U. De Sanctis ${ }^{135 a, 135 b}$, A. De Santo ${ }^{151}$, K. De Vasconcelos Corga ${ }^{88}$, J.B. De Vivie De Regie ${ }^{119}$, W.J. Dearnaley ${ }^{75}$, R. Debbe ${ }^{27}$, C. Debenedetti ${ }^{139}$, D.V. Dedovich ${ }^{68}$, N. Dehghanian ${ }^{3}$, I. Deigaard ${ }^{109}$, M. Del Gaudio ${ }^{40 a, 40 b}$, J. Del Peso ${ }^{85}$, T. Del Prete ${ }^{126 a, 126 b}$, D. Delgove ${ }^{119}$, F. Deliot ${ }^{138}$, C.M. Delitzsch ${ }^{52}$, A. Dell'Acqua ${ }^{32}$, L. Dell'Asta ${ }^{24}$, M. Dell'Orso ${ }^{126 a, 126 b}$, M. Della Pietra ${ }^{106 a, 106 b}$, D. della Volpe ${ }^{52}$, M. Delmastro ${ }^{5}$, C. Delporte ${ }^{119}$, P.A. Delsart ${ }^{58}$, D.A. DeMarco ${ }^{161}$, S. Demers ${ }^{179}$, M. Demichev ${ }^{68}$, A. Demilly ${ }^{83}$, S.P. Denisov ${ }^{132}$, D. Denysiuk ${ }^{138}$, D. Derendarz ${ }^{42}$, J.E. Derkaoui ${ }^{137 d}$, F. Derue ${ }^{83}$, P. Dervan ${ }^{77}$, K. Desch ${ }^{23}$, C. Deterre ${ }^{45}$, K. Dette ${ }^{46}$, M.R. Devesa ${ }^{29}$, P.O. Deviveiros ${ }^{32}$, A. Dewhurst ${ }^{133}$, S. Dhaliwal ${ }^{25}$, F.A. Di Bello ${ }^{52}$, A. Di Ciaccio ${ }^{135 a, 135 b}$, L. Di Ciaccio ${ }^{5}$, W.K. Di Clemente ${ }^{124}$, C. Di Donato ${ }^{106 a, 106 b}$, A. Di Girolamo ${ }^{32}$, B. Di Girolamo ${ }^{32}$, B. Di Micco ${ }^{136 a, 136 b}$, R. Di Nardo ${ }^{32}$, K.F. Di Petrillo ${ }^{59}$, A. Di Simone ${ }^{51}$, R. Di Sipio ${ }^{161}$, D. Di Valentino ${ }^{31}$, C. Diaconu ${ }^{88}$, M. Diamond ${ }^{161}$, F.A. Dias $^{39}$, M.A. Diaz ${ }^{34 a}$, E.B. Diehl ${ }^{92}$, J. Dietrich ${ }^{17}$, S. Díez Cornell ${ }^{45}$,

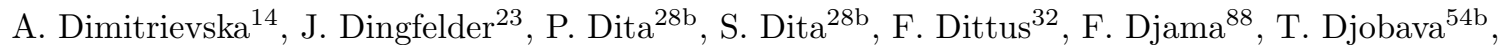
J.I. Djuvsland ${ }^{60 a}$, M.A.B. do Vale ${ }^{26 c}$, D. Dobos ${ }^{32}$, M. Dobre ${ }^{28 b}$, C. Doglioni ${ }^{84}$, J. Dolejsi ${ }^{131}$, Z. Dolezal ${ }^{131}$, M. Donadelli26d, S. Donati ${ }^{126 a, 126 b}$, P. Dondero ${ }^{123 a, 123 b}$, J. Donini ${ }^{37}$, J. Dopke ${ }^{133}$, A. Doria ${ }^{106 a}$, M.T. Dova ${ }^{74}$, A.T. Doyle ${ }^{56}$, E. Drechsler ${ }^{57}$, M. Dris ${ }^{10}$, Y. Du ${ }^{36 b}$, J. Duarte-Campderros ${ }^{155}$, A. Dubreuil ${ }^{52}$, E. Duchovni ${ }^{175}$, G. Duckeck ${ }^{102}$, A. Ducourthial ${ }^{83}$, O.A. Ducu ${ }^{97, p}$, D. Duda ${ }^{109}$, A. Dudarev ${ }^{32}$, A.Chr. Dudder ${ }^{86}$, E.M. Duffield ${ }^{16}$, L. Duflot ${ }^{119}$, M. Dührssen ${ }^{32}$, M. Dumancic ${ }^{175}$, A.E. Dumitriu ${ }^{28 b}$, A.K. Duncan $^{56}$, M. Dunford ${ }^{60 a}$, H. Duran Yildiz ${ }^{4 a}$, M. Düren ${ }^{55}$, A. Durglishvili ${ }^{54 b}$, D. Duschinger ${ }^{47}$, B. Dutta ${ }^{45}$, M. Dyndal $^{45}$, C. Eckardt ${ }^{45}$, K.M. Ecker ${ }^{103}$, R.C. Edgar ${ }^{92}$, T. Eifert ${ }^{32}$, G. Eigen $^{15}$, K. Einsweiler ${ }^{16}$, T. Ekelof ${ }^{168}$, M. El Kacimi ${ }^{137 c}$, R. El Kosseifi ${ }^{88}$, V. Ellajosyula ${ }^{88}$, M. Ellert $^{168}$, S. Elles ${ }^{5}$, F. Ellinghaus ${ }^{178}$, A.A. Elliot $^{172}$, N. Ellis ${ }^{32}$, J. Elmsheuser ${ }^{27}$, M. Elsing ${ }^{32}$, D. Emeliyanov ${ }^{133}$, Y. Enari ${ }^{157}$, O.C. Endner ${ }^{86}$, J.S. Ennis ${ }^{173}$, J. Erdmann ${ }^{46}$, A. Ereditato ${ }^{18}$, G. Ernis ${ }^{178}$, M. Ernst ${ }^{27}$, S. Errede ${ }^{169}$, M. Escalier ${ }^{119}$, C. Escobar ${ }^{127}$, B. Esposito ${ }^{50}$, O. Estrada Pastor $^{170}$, A.I. Etienvre ${ }^{138}$, E. Etzion ${ }^{155}$, H. Evans ${ }^{64}$, A. Ezhilov ${ }^{125}$, M. Ezzi ${ }^{137 e}$, F. Fabbri ${ }^{22 a, 22 b}$, L. Fabbrii ${ }^{22 a, 22 b}$, G. Facini ${ }^{33}$, R.M. Fakhrutdinov ${ }^{132}$, S. Falciano ${ }^{134 a}$, R.J. Falla ${ }^{81}$, J. Faltova ${ }^{32}$, Y. Fang ${ }^{35 a}$, M. Fanti ${ }^{94 a, 94 b}$, A. Farbin ${ }^{8}$, A. Farilla ${ }^{136 a}$, C. Farina ${ }^{127}$, E.M. Farina ${ }^{123 a, 123 b}$, T. Farooque ${ }^{93}$, S. Farrell ${ }^{16}$, S.M. Farrington ${ }^{173}$, P. Farthouat ${ }^{32}$, F. Fassi ${ }^{137 e}$, P. Fassnacht ${ }^{32}$, D. Fassouliotis ${ }^{9}$, M. Faucci Giannelli ${ }^{80}$, A. Favareto ${ }^{53 a, 53 b}$, W.J. Fawcett ${ }^{122}$, L. Fayard ${ }^{119}$, O.L. Fedin ${ }^{125, q}$, W. Fedorko ${ }^{171}$, S. Feigl ${ }^{121}$, L. Feligioni ${ }^{88}$, C. Feng ${ }^{36 b}$, E.J. Feng ${ }^{32}$, H. Feng ${ }^{92}$, M.J. Fenton ${ }^{56}$, A.B. Fenyuk ${ }^{132}$, L. Feremenga ${ }^{8}$, P. Fernandez Martinez ${ }^{170}$, S. Fernandez Perez ${ }^{13}$, J. Ferrando ${ }^{45}$, A. Ferrari ${ }^{168}$, P. Ferrari ${ }^{109}$, R. Ferrari ${ }^{123 a}$, D.E. Ferreira de Lima ${ }^{60 b}$, A. Ferrer ${ }^{170}$, D. Ferrere ${ }^{52}$, C. Ferretti ${ }^{92}$, F. Fiedler ${ }^{86}$, A. Filipčič ${ }^{78}$, M. Filipuzzi ${ }^{45}$, F. Filthaut ${ }^{108}$, M. Fincke-Keeler ${ }^{172}$, K.D. Finelli ${ }^{152}$, M.C.N. Fiolhais ${ }^{128 a, 128 c, r}$, L. Fiorini ${ }^{170}$, A. Fischer ${ }^{2}$, C. Fischer ${ }^{13}$, J. Fischer ${ }^{178}$, W.C. Fisher ${ }^{93}$, N. Flaschel ${ }^{45}$, I. Fleck ${ }^{143}$, P. Fleischmann ${ }^{92}$, R.R.M. Fletcher ${ }^{124}$, T. Flick ${ }^{178}$, 
B.M. Flierl ${ }^{102}$, L.R. Flores Castillo ${ }^{62 a}$, M.J. Flowerdew ${ }^{103}$, G.T. Forcolin ${ }^{87}$, A. Formica ${ }^{138}$, F.A. Förster ${ }^{13}$, A. Forti ${ }^{87}$, A.G. Foster ${ }^{19}$, D. Fournier ${ }^{119}$, H. Fox ${ }^{75}$, S. Fracchia ${ }^{141}$, P. Francavilla ${ }^{83}$, M. Franchini ${ }^{22 a, 22 b}$, S. Franchino ${ }^{60 a}$, D. Francis $^{32}$, L. Franconi ${ }^{121}$, M. Franklin ${ }^{59}$, M. Frate ${ }^{166}$, M. Fraternali ${ }^{123 a, 123 b}$, D. Freeborn ${ }^{81}$, S.M. Fressard-Batraneanu ${ }^{32}$, B. Freund ${ }^{97}$, D. Froidevaux ${ }^{32}$, J.A. Frost ${ }^{122}$, C. Fukunaga ${ }^{158}$, T. Fusayasu ${ }^{104}$, J. Fuster ${ }^{170}$, C. Gabaldon ${ }^{58}$, O. Gabizon ${ }^{154}$, A. Gabriellii2a,22b, A. Gabrielli ${ }^{16}$, G.P. Gach ${ }^{41 a}$, S. Gadatsch ${ }^{32}$, S. Gadomski ${ }^{80}$, G. Gagliardi ${ }^{53 a, 53 b}$, L.G. Gagnon ${ }^{97}$, C. Galea ${ }^{108}$, B. Galhardo ${ }^{128 a, 128 c}$, E.J. Gallas ${ }^{122}$, B.J. Gallop ${ }^{133}$, P. Gallus ${ }^{130}$, G. Galster ${ }^{39}$, K.K. Gan ${ }^{113}$, S. Ganguly ${ }^{37}$, Y. Gao ${ }^{77}$, Y.S. Gao ${ }^{145, g}$, F.M. Garay Walls ${ }^{49}$, C. García ${ }^{170}$, J.E. García Navarro ${ }^{170}$, M. Garcia-Sciveres ${ }^{16}$, R.W. Gardner ${ }^{33}$, N. Garelli ${ }^{145}$, V. Garonne ${ }^{121}$, A. Gascon Bravo ${ }^{45}$, K. Gasnikova ${ }^{45}$, C. Gatti ${ }^{50}$, A. Gaudiello ${ }^{53 a, 53 b}$, G. Gaudio ${ }^{123 a}$, I.L. Gavrilenko ${ }^{98}$, C. Gay ${ }^{171}$, G. Gaycken ${ }^{23}$, E.N. Gazis ${ }^{10}$, C.N.P. Gee ${ }^{133}$, J. Geisen ${ }^{57}$, M. Geisen ${ }^{86}$, M.P. Geisler ${ }^{60 a}$, K. Gellerstedt ${ }^{148 a, 148 b}$, C. Gemme ${ }^{53 a}$, M.H. Genest ${ }^{58}$, C. Geng ${ }^{92}$, S. Gentile ${ }^{134 a, 134 b}$, C. Gentsos ${ }^{156}$, S. George ${ }^{80}$, D. Gerbaudo ${ }^{13}$, A. Gershon ${ }^{155}$, G. Geßner ${ }^{46}$, S. Ghasemi ${ }^{143}$, M. Ghneimat ${ }^{23}$, B. Giacobbe ${ }^{22 a}$, S. Giagu ${ }^{134 a, 134 b}$, P. Giannetti ${ }^{126 a, 126 b}$, S.M. Gibson ${ }^{80}$, M. Gignac ${ }^{171}$, M. Gilchriese ${ }^{16}$, D. Gillberg ${ }^{31}$, G. Gilles ${ }^{178}$, D.M. Gingrich ${ }^{3, d}$, N. Giokaris ${ }^{9, *}$, M.P. Giordani ${ }^{167 a, 167 c}$, F.M. Giorgii ${ }^{2 a}$, P.F. Giraud ${ }^{138}$, P. Giromini ${ }^{59}$, D. Giugni ${ }^{94 a}$, F. Giuli ${ }^{122}$, C. Giuliani ${ }^{103}$, M. Giulini ${ }^{60 b}$, B.K. Gjelsten ${ }^{121}$, S. Gkaitatzis ${ }^{156}$, I. Gkialas ${ }^{9}$, E.L. Gkougkousis ${ }^{139}$, P. Gkountoumis ${ }^{10}$, L.K. Gladilin ${ }^{101}$, C. Glasman ${ }^{85}$, J. Glatzer ${ }^{13}$, P.C.F. Glaysher ${ }^{45}$, A. Glazov ${ }^{45}$, M. Goblirsch-Kolb ${ }^{25}$, J. Godlewski ${ }^{42}$, S. Goldfarb ${ }^{91}$, T. Golling ${ }^{52}$, D. Golubkov ${ }^{132}$, A. Gomes ${ }^{128 a, 128 b, 128 d}$, R. GonC calo $^{128 a}$, R. Goncalves Gama ${ }^{26 a}$, J. Goncalves Pinto Firmino Da Costa ${ }^{138}$, G. Gonella ${ }^{51}$, L. Gonella ${ }^{19}$, A. Gongadze ${ }^{68}$, S. González de la $\mathrm{Hoz}^{170}$, S. Gonzalez-Sevilla ${ }^{52}$, L. Goossens ${ }^{32}$, P.A. Gorbounov ${ }^{99}$, H.A. Gordon ${ }^{27}$, I. Gorelov ${ }^{107}$, B. Gorini ${ }^{32}$, E. Gorini ${ }^{76 a, 76 b}$, A. Gorišek ${ }^{78}$, A.T. Goshaw ${ }^{48}$, C. Gössling ${ }^{46}$, M.I. Gostkin ${ }^{68}$, C.A. Gottardo ${ }^{23}$, C.R. Goudet ${ }^{119}$, D. Goujdami ${ }^{137 c}$, A.G. Goussiou ${ }^{140}$, N. Govender ${ }^{147 b, s}$, E. Gozani ${ }^{154}$, L. Graber ${ }^{57}$, I. Grabowska-Bold ${ }^{41 a}$, P.O.J. Gradin ${ }^{168}$, J. Gramling ${ }^{166}$, E. Gramstad ${ }^{121}$, S. Grancagnolo ${ }^{17}$, V. Gratchev ${ }^{125}$, P.M. Gravila ${ }^{28 f}$, C. Gray ${ }^{56}$, H.M. Gray ${ }^{16}$, Z.D. Greenwood ${ }^{82, t}$, C. Grefe ${ }^{23}$, K. Gregersen ${ }^{81}$, I.M. Gregor ${ }^{45}$, P. Grenier ${ }^{145}$, K. Grevtsov ${ }^{5}$, J. Griffiths ${ }^{8}$, A.A. Grillo ${ }^{139}$, K. Grimm ${ }^{75}$, S. Grinstein ${ }^{13, u}$, Ph. Gris ${ }^{37}$, J.-F. Grivaz ${ }^{119}$, S. Groh ${ }^{86}$, E. Gross ${ }^{175}$, J. Grosse-Knetter ${ }^{57}$, G.C. Grossi ${ }^{82}$, Z.J. Grout ${ }^{81}$, A. Grummer ${ }^{107}$, L. Guan ${ }^{92}$, W. Guan ${ }^{176}$, J. Guenther ${ }^{65}$, F. Guescini ${ }^{163 a}$, D. Guest ${ }^{166}$, O. Gueta ${ }^{155}$, B. Gui ${ }^{113}$, E. Guido ${ }^{53 a, 53 b}$,

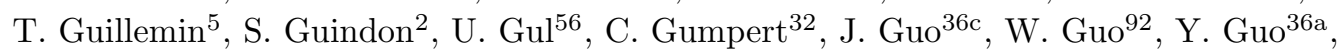

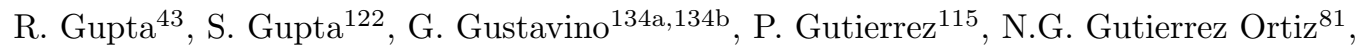
C. Gutschow ${ }^{81}$, C. Guyot ${ }^{138}$, M.P. Guzik ${ }^{41 a}$, C. Gwenlan ${ }^{122}$, C.B. Gwilliam ${ }^{77}$, A. Haas ${ }^{12}$, C. Haber ${ }^{16}$, H.K. Hadavand ${ }^{8}$, N. Haddad ${ }^{137 e}$, A. Hadef ${ }^{88}$, S. Hageböck ${ }^{23}$, M. Hagihara ${ }^{164}$, H. Hakobyan ${ }^{180, *}$, M. Haleem ${ }^{45}$, J. Haley ${ }^{116}$, G. Halladjian ${ }^{93}$, G.D. Hallewell ${ }^{88}$, K. Hamacher ${ }^{178}$, P. Hamal ${ }^{117}$, K. Hamano ${ }^{172}$, A. Hamilton ${ }^{147 a}$, G.N. Hamity ${ }^{141}$, P.G. Hamnett ${ }^{45}$, L. Han ${ }^{36 a}$, S. $\operatorname{Han}^{35 a}$, K. Hanagaki ${ }^{69, v}$, K. Hanawa ${ }^{157}$, M. Hance ${ }^{139}$, B. Haney ${ }^{124}$, P. Hanke ${ }^{60 a}$, J.B. Hansen ${ }^{39}$, J.D. Hansen ${ }^{39}$, M.C. Hansen ${ }^{23}$, P.H. Hansen ${ }^{39}$, K. Hara ${ }^{164}$, A.S. Hard ${ }^{176}$, T. Harenberg ${ }^{178}$, F. Hariri ${ }^{119}$, S. Harkusha ${ }^{95}$, R.D. Harrington ${ }^{49}$, P.F. Harrison ${ }^{173}$, F. Hartjes ${ }^{109}$, N.M. Hartmann ${ }^{102}$, M. Hasegawa ${ }^{70}$, Y. Hasegawa ${ }^{142}$, A. Hasib ${ }^{49}$, S. Hassani ${ }^{138}$, S. Haug ${ }^{18}$, R. Hauser ${ }^{93}$, L. Hauswald ${ }^{47}$, L.B. Havener ${ }^{38}$, M. Havranek ${ }^{130}$, C.M. Hawkes ${ }^{19}$, R.J. Hawkings ${ }^{32}$, D. Hayakawa ${ }^{159}$, D. Hayden ${ }^{93}$, C.P. Hays ${ }^{122}$, J.M. Hays ${ }^{79}$, H.S. Hayward ${ }^{77}$, S.J. Haywood ${ }^{133}$,

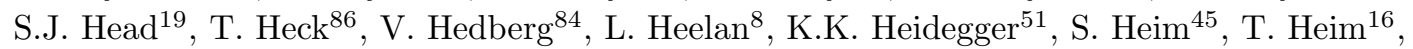
B. Heinemann ${ }^{45, w}$, J.J. Heinrich ${ }^{102}$, L. Heinrich ${ }^{112}$, C. Heinz ${ }^{55}$, J. Hejbal ${ }^{129}$, L. Helary ${ }^{32}$, A. Held ${ }^{171}$, S. Hellman ${ }^{148 a, 148 b}$, C. Helsens ${ }^{32}$, R.C.W. Henderson ${ }^{75}$, Y. Heng ${ }^{176}$,

S. Henkelmann ${ }^{171}$, A.M. Henriques Correia ${ }^{32}$, S. Henrot-Versille ${ }^{119}$, G.H. Herbert ${ }^{17}$, H. Herde ${ }^{25}$, V. Herget ${ }^{177}$, Y. Hernández Jiménez ${ }^{147 c}$, G. Herten ${ }^{51}$, R. Hertenberger ${ }^{102}$, L. Hervas ${ }^{32}$, 
T.C. Herwig ${ }^{124}$, G.G. Hesketh ${ }^{81}$, N.P. Hessey ${ }^{163 a}$, J.W. Hetherly ${ }^{43}$, S. Higashino ${ }^{69}$, E. Higón-Rodriguez ${ }^{170}$, E. Hill ${ }^{172}$, J.C. Hill ${ }^{30}$, K.H. Hiller ${ }^{45}$, S.J. Hillier ${ }^{19}$, M. Hils ${ }^{47}$, I. Hinchliffe ${ }^{16}$, M. Hirose ${ }^{51}$, D. Hirschbuehl ${ }^{178}$, B. Hiti ${ }^{78}$, O. Hladik ${ }^{129}$, X. Hoad ${ }^{49}$, J. Hobbs ${ }^{150}$, N. Hod ${ }^{163 a}$, M.C. Hodgkinson ${ }^{141}$, P. Hodgson ${ }^{141}$, A. Hoecker ${ }^{32}$, M.R. Hoeferkamp ${ }^{107}$, F. Hoenig ${ }^{102}$, D. Hohn ${ }^{23}$, T.R. Holmes ${ }^{33}$, M. Homann ${ }^{46}$, S. Honda ${ }^{164}$, T. Honda ${ }^{69}$, T.M. Hong ${ }^{127}$, B.H. Hooberman ${ }^{169}$, W.H. Hopkins ${ }^{118}$, Y. Horii ${ }^{105}$, A.J. Horton ${ }^{144}$, J-Y. Hostachy ${ }^{58}$, S. Hou ${ }^{153}$, A. Hoummada ${ }^{137 a}$, J. Howarth ${ }^{45}$, J. Hoya ${ }^{74}$, M. Hrabovsky ${ }^{117}$, J. Hrdinka ${ }^{32}$, I. Hristova ${ }^{17}$, J. Hrivnac ${ }^{119}$, T. Hryn'ova ${ }^{5}$, A. Hrynevich ${ }^{96}$, P.J. $\mathrm{Hsu}^{63}$, S.-C. $\mathrm{Hsu}^{140}$, Q. Hu ${ }^{36 a}$, S. Hu ${ }^{36 c}$, Y. Huang ${ }^{35 a}$, Z. Hubacek ${ }^{130}$, F. Hubaut ${ }^{88}$, F. Huegging ${ }^{23}$, T.B. Huffman ${ }^{122}$, E.W. Hughes ${ }^{38}$,

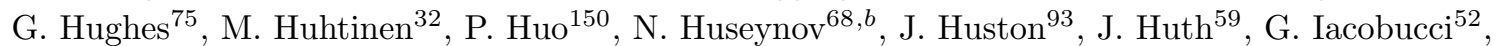
G. Iakovidis ${ }^{27}$, I. Ibragimov ${ }^{143}$, L. Iconomidou-Fayard ${ }^{119}$, Z. Idrissi ${ }^{137 e}$, P. Iengo ${ }^{32}$, O. Igonkina ${ }^{109, x}$, T. Iizawa ${ }^{174}$, Y. Ikegami ${ }^{69}$, M. Ikeno ${ }^{69}$, Y. Ilchenko ${ }^{11, y}$, D. Iliadis ${ }^{156}$, N. Ilic ${ }^{145}$,

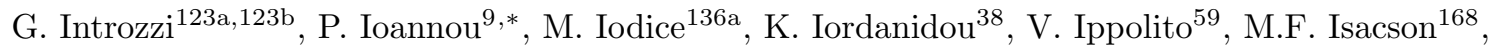

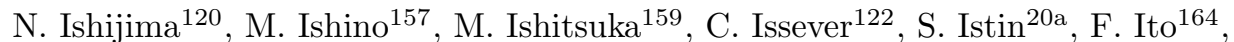
J.M. Iturbe Ponce ${ }^{87}$, R. Iuppa ${ }^{162 a, 162 b}$, H. Iwasaki ${ }^{69}$, J.M. Izen $^{44}$, V. Izzo ${ }^{106 a}$, S. Jabbar ${ }^{3}$, P. Jackson ${ }^{1}$, R.M. Jacobs ${ }^{23}$, V. Jain ${ }^{2}$, K.B. Jakobi ${ }^{86}$, K. Jakobs ${ }^{51}$, S. Jakobsen ${ }^{65}$, T. Jakoubek ${ }^{129}$, D.O. Jamin ${ }^{116}$, D.K. Jana ${ }^{82}$, R. Jansky ${ }^{52}$, J. Janssen ${ }^{23}$, M. Janus ${ }^{57}$, P.A. Janus ${ }^{41 a}$, G. Jarlskog ${ }^{84}$, N. Javadov ${ }^{68, b}$, T. Javurek ${ }^{51}$, M. Javurkova ${ }^{51}$, F. Jeanneau ${ }^{138}$, L. Jeanty ${ }^{16}$, J. Jejelava ${ }^{54 a, z}$,

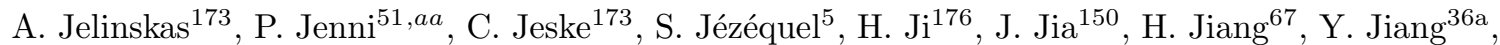
Z. Jiang ${ }^{145}$, S. Jiggins ${ }^{81}$, J. Jimenez Pena ${ }^{170}$, S. Jin ${ }^{35 a}$, A. Jinaru ${ }^{28 b}$, O. Jinnouchi ${ }^{159}$, H. Jivan ${ }^{147 c}$, P. Johansson ${ }^{141}$, K.A. Johns ${ }^{7}$, C.A. Johnson ${ }^{64}$, W.J. Johnson ${ }^{140}$, K. Jon-And ${ }^{148 a, 148 b}$, R.W.L. Jones ${ }^{75}$, S.D. Jones ${ }^{151}$, S. Jones ${ }^{7}$, T.J. Jones ${ }^{77}$, J. Jongmanns ${ }^{60 a}$, P.M. Jorge ${ }^{128 a, 128 b}$, J. Jovicevic ${ }^{163 a}$, X. Ju${ }^{176}$, A. Juste Rozas ${ }^{13, u}$, M.K. Köhler ${ }^{175}$, A. Kaczmarska ${ }^{42}$, M. Kado ${ }^{119}$, H. Kagan ${ }^{113}$, M. Kagan ${ }^{145}$, S.J. Kahn ${ }^{88}$, T. Kaji1 ${ }^{174}$, E. Kajomovitz ${ }^{48}$, C.W. Kalderon ${ }^{84}$, A. Kaluza ${ }^{86}$, S. Kama ${ }^{43}$, A. Kamenshchikov ${ }^{132}$, N. Kanaya ${ }^{157}$, L. Kanjir ${ }^{78}$, V.A. Kantserov ${ }^{100}$, J. Kanzaki ${ }^{69}$, B. Kaplan ${ }^{112}$, L.S. Kaplan ${ }^{176}$, D. $\operatorname{Kar}^{147 c}$, K. Karakostas ${ }^{10}$, N. Karastathis ${ }^{10}$, M.J. Kareem ${ }^{57}$, E. Karentzos ${ }^{10}$, S.N. Karpov ${ }^{68}$, Z.M. Karpova ${ }^{68}$, K. Karthik ${ }^{112}$, V. Kartvelishvili ${ }^{75}$, A.N. Karyukhin ${ }^{132}$, K. Kasahara ${ }^{164}$, L. Kashif ${ }^{176}$, R.D. Kass ${ }^{113}$, A. Kastanas ${ }^{149}$, Y. Kataoka ${ }^{157}$, C. Kato ${ }^{157}$, A. Katre ${ }^{52}$, J. Katzy ${ }^{45}$, K. Kawade ${ }^{70}$, K. Kawagoe ${ }^{73}$, T. Kawamoto ${ }^{157}$, G. Kawamura ${ }^{57}$, E.F. Kay ${ }^{77}$, V.F. Kazanin ${ }^{111, c}$, R. Keeler ${ }^{172}$, R. Kehoe ${ }^{43}$, J.S. Keller ${ }^{31}$, J.J. Kempster ${ }^{80}$, J Kendrick ${ }^{19}$, H. Keoshkerian ${ }^{161}$, O. Kepka ${ }^{129}$, B.P. Kerševan ${ }^{78}$, S. Kersten ${ }^{178}$, R.A. Keyes ${ }^{90}$, M. Khader ${ }^{169}$, F. Khalil-zada ${ }^{12}$, A. Khanov ${ }^{116}$, A.G. Kharlamov ${ }^{111, c}$, T. Kharlamova ${ }^{111, c}$, A. Khodinov ${ }^{160}$, T.J. Khoo ${ }^{52}$, V. Khovanskiy ${ }^{99, *}$, E. Khramov ${ }^{68}$, J. Khubua ${ }^{54 b, a b}$, S. Kido ${ }^{70}$, C.R. Kilby ${ }^{80}$, H.Y. Kim ${ }^{8}$, S.H. Kim ${ }^{164}$, Y.K. Kim $^{33}$, N. Kimura ${ }^{156}$, O.M. Kind ${ }^{17}$, B.T. King 77 , D. Kirchmeier ${ }^{47}$, J. Kirk ${ }^{133}$, A.E. Kiryunin ${ }^{103}$, T. Kishimoto ${ }^{157}$, D. Kisielewska ${ }^{41 a}$, K. Kiuchi ${ }^{164}$, O. Kivernyk ${ }^{5}$, E. Kladiva ${ }^{146 b}$, T. Klapdor-Kleingrothaus ${ }^{51}$, M.H. Klein ${ }^{38}$, M. Klein ${ }^{77}$, U. Klein ${ }^{77}$, K. Kleinknecht ${ }^{86}$, P. Klimek ${ }^{110}$, A. Klimentov ${ }^{27}$, R. Klingenberg ${ }^{46}$, T. Kling ${ }^{23}$, T. Klioutchnikova ${ }^{32}$, E.-E. Kluge ${ }^{60 a}$, P. Kluit ${ }^{109}$, S. Kluth ${ }^{103}$, E. Kneringer ${ }^{65}$, E.B.F.G. Knoops ${ }^{88}$, A. Knue ${ }^{103}$, A. Kobayashi ${ }^{157}$, D. Kobayashi ${ }^{159}$, T. Kobayashi ${ }^{157}$, M. Kobel ${ }^{47}$, M. Kocian ${ }^{145}$, P. Kodys ${ }^{131}$, T. Koffas ${ }^{31}$, E. Koffeman ${ }^{109}$, N.M. Köhler ${ }^{103}$, T. Koi ${ }^{145}$, M. Kolb ${ }^{60 b}$, I. Koletsou ${ }^{5}$, A.A. Komar ${ }^{98, *}$, Y. Komori ${ }^{157}$, T. Kondo ${ }^{69}$, N. Kondrashova ${ }^{36 c}$, K. Köneke ${ }^{51}$, A.C. König ${ }^{108}$, T. Kono ${ }^{69, a c}$, R. Konoplich ${ }^{112, a d}$, N. Konstantinidis ${ }^{81}$, R. Kopeliansky ${ }^{64}$, S. Koperny ${ }^{41 a}$, A.K. Kopp ${ }^{51}$, K. Korcyl ${ }^{42}$, K. Kordas ${ }^{156}$, A. Korn ${ }^{81}$, A.A. Korol ${ }^{111, c}$, I. Korolkov ${ }^{13}$, E.V. Korolkova ${ }^{141}$, O. Kortner ${ }^{103}$, S. Kortner ${ }^{103}$, T. Kosek ${ }^{131}$, V.V. Kostyukhin ${ }^{23}$, A. Kotwal ${ }^{48}$, A. Koulouris ${ }^{10}$, A. Kourkoumeli-Charalampidi ${ }^{123 a, 123 b}$, C. Kourkoumelis ${ }^{9}$, E. Kourlitis ${ }^{141}$, V. Kouskoura ${ }^{27}$, A.B. Kowalewska ${ }^{42}$, R. Kowalewski ${ }^{172}$, T.Z. Kowalski ${ }^{41 a}$, C. Kozakai $^{157}$, W. Kozanecki ${ }^{138}$, A.S. Kozhin ${ }^{132}$, V.A. Kramarenko ${ }^{101}$, G. Kramberger ${ }^{78}$, D. Krasnopevtsev ${ }^{100}$, M.W. Krasny ${ }^{83}$, 
A. Krasznahorkay ${ }^{32}$, D. Krauss ${ }^{103}$, J.A. Kremer ${ }^{41 a}$, J. Kretzschmar ${ }^{77}$, K. Kreutzfeldt ${ }^{55}$, P. Krieger ${ }^{161}$, K. Krizka ${ }^{33}$, K. Kroeninger ${ }^{46}$, H. Kroha ${ }^{103}$, J. Kroll ${ }^{129}$, J. Kroll ${ }^{124}$, J. Kroseberg ${ }^{23}$, J. Krstic ${ }^{14}$, U. Kruchonak ${ }^{68}$, H. Krüger ${ }^{23}$, N. Krumnack ${ }^{67}$, M.C. Kruse ${ }^{48}$, T. Kubota ${ }^{91}$, H. Kucuk ${ }^{81}$, S. Kuday ${ }^{4 b}$, J.T. Kuechler ${ }^{178}$, S. Kuehn ${ }^{32}$, A. Kugel ${ }^{60 c}$, F. Kuger ${ }^{177}$, T. Kuhl ${ }^{45}$, V. Kukhtin ${ }^{68}$, R. Kukla ${ }^{88}$, Y. Kulchitsky ${ }^{95}$, S. Kuleshov ${ }^{34 b}$, Y.P. Kulinich ${ }^{169}$, M. Kuna ${ }^{134 a, 134 b}$, T. Kunigo ${ }^{71}$, A. Kupco ${ }^{129}$, T. Kupfer ${ }^{46}$, O. Kuprash ${ }^{155}$, H. Kurashige ${ }^{70}$, L.L. Kurchaninov ${ }^{163 a}$, Y.A. Kurochkin ${ }^{95}$, M.G. Kurth ${ }^{35 a}$, V. Kus ${ }^{129}$, E.S. Kuwertz ${ }^{172}$, M. Kuze ${ }^{159}$, J. Kvita ${ }^{117}$, T. Kwan ${ }^{172}$, D. Kyriazopoulos ${ }^{141}$, A. La Rosa ${ }^{103}$, J.L. La Rosa Navarro ${ }^{26 d}$, L. La Rotonda ${ }^{40 a, 40 b}$, C. Lacasta ${ }^{170}$, F. Lacava ${ }^{134 a, 134 b}$, J. Lacey ${ }^{45}$, H. Lacker ${ }^{17}$, D. Lacour ${ }^{83}$, E. Ladygin ${ }^{68}$, R. Lafaye ${ }^{5}$, B. Laforge ${ }^{83}$, T. Lagouri ${ }^{179}$, S. Lai ${ }^{57}$, S. Lammers ${ }^{64}$, W. Lampl ${ }^{7}$, E. LanC con $^{27}$, U. Landgraf ${ }^{51}$, M.P.J. Landon ${ }^{79}$, M.C. Lanfermann ${ }^{52}$, V.S. Lang ${ }^{60 a}$, J.C. Lange ${ }^{13}$, R.J. Langenberg ${ }^{32}$, A.J. Lankford ${ }^{166}$, F. Lanni ${ }^{27}$, K. Lantzsch ${ }^{23}$, A. Lanza ${ }^{123 a}$, A. Lapertosa ${ }^{53 a, 53 b}$, S. Laplace ${ }^{83}$,

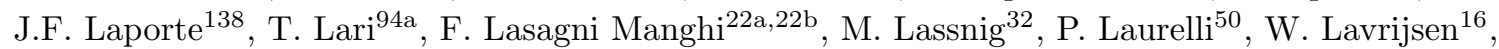

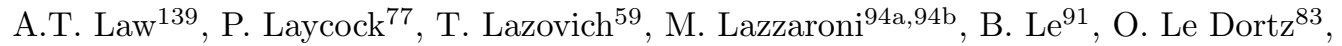
E. Le Guirriec ${ }^{88}$, E.P. Le Quilleuc ${ }^{138}$, M. LeBlanc ${ }^{172}$, T. LeCompte $^{6}$, F. Ledroit-Guillon ${ }^{58}$, C.A. Lee ${ }^{27}$, G.R. Lee ${ }^{133, a e}$, S.C. Lee ${ }^{153}$, L. Lee ${ }^{59}$, B. Lefebvre ${ }^{90}$, G. Lefebvre ${ }^{83}$, M. Lefebvre ${ }^{172}$, F. Legger ${ }^{102}$, C. Leggett ${ }^{16}$, A. Lehan ${ }^{77}$, G. Lehmann Miotto ${ }^{32}$, X. Lei ${ }^{7}$, W.A. Leight ${ }^{45}$, M.A.L. Leite ${ }^{26 \mathrm{~d}}$, R. Leitner ${ }^{131}$, D. Lellouch ${ }^{175}$, B. Lemmer ${ }^{57}$, K.J.C. Leney ${ }^{81}$, T. Lenz ${ }^{23}$, B. Lenzi ${ }^{32}$, R. Leone ${ }^{7}$, S. Leone ${ }^{126 a, 126 b}$, C. Leonidopoulos ${ }^{49}$, G. Lerner ${ }^{151}$, C. Leroy ${ }^{97}$, A.A.J. Lesage ${ }^{138}$, C.G. Lester ${ }^{30}$, M. Levchenko ${ }^{125}$, J. Levêque ${ }^{5}$, D. Levin ${ }^{92}$, L.J. Levinson ${ }^{175}$,

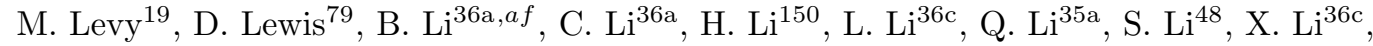

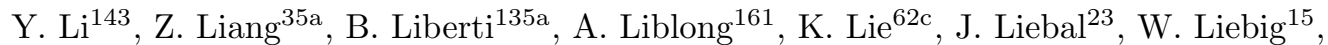
A. Limosani ${ }^{152}$, S.C. $\operatorname{Lin}^{183}$, T.H. $\operatorname{Lin}^{86}$, B.E. Lindquist ${ }^{150}$, A.E. Lionti ${ }^{52}$, E. Lipeles ${ }^{124}$, A. Lipniacka ${ }^{15}$, M. Lisovyi ${ }^{60 b}$, T.M. Liss ${ }^{169}$, A. Lister ${ }^{171}$, A.M. Litke ${ }^{139}$, B. Liu ${ }^{153, a g}$, H. Liu ${ }^{92}$,

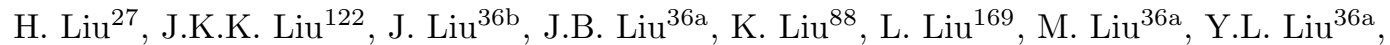
Y. Liu ${ }^{36 a}$, M. Livan ${ }^{123 a, 123 b}$, A. Lleres ${ }^{58}$, J. Llorente Merino ${ }^{35 a}$, S.L. Lloyd ${ }^{79}$, C.Y. Lo ${ }^{62 b}$, F. Lo Sterzo ${ }^{153}$, E.M. Lobodzinska ${ }^{45}$, P. Loch ${ }^{7}$, F.K. Loebinger ${ }^{87}$, A. Loesle ${ }^{51}$, K.M. Loew ${ }^{25}$, A. Loginov ${ }^{179, *}$, T. Lohse $^{17}$, K. Lohwasser ${ }^{45}$, M. Lokajicek ${ }^{129}$, B.A. Long ${ }^{24}$, J.D. Long ${ }^{169}$, R.E. Long ${ }^{75}$, L. Longo ${ }^{76 a, 76 b}$, K.A. Looper ${ }^{113}$, J.A. Lopez ${ }^{34 b}$, D. Lopez Mateos ${ }^{59}$, I. Lopez Paz ${ }^{13}$, A. Lopez Solis ${ }^{83}$, J. Lorenz ${ }^{102}$, N. Lorenzo Martinez ${ }^{5}$, M. Losada ${ }^{21}$, P.J. Lösel ${ }^{102}$, X. Lou ${ }^{35 a}$,

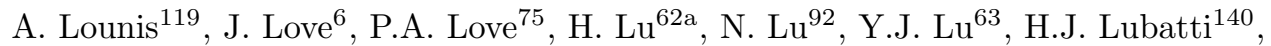
C. Luci ${ }^{134 a, 134 b}$, A. Lucotte ${ }^{58}$, C. Luedtke ${ }^{51}$, F. Luehring ${ }^{64}$, W. Lukas ${ }^{65}$, L. Luminari ${ }^{134 a}$, O. Lundberg ${ }^{148 a, 148 b}$, B. Lund-Jensen ${ }^{149}$, P.M. Luzi ${ }^{83}$, D. Lynn ${ }^{27}$, R. Lysak ${ }^{129}$, E. Lytken ${ }^{84}$, V. Lyubushkin ${ }^{68}$, H. Ma ${ }^{27}$, L.L. Ma ${ }^{36 \mathrm{~b}}$, Y. Ma ${ }^{36 \mathrm{~b}}$, G. Maccarrone ${ }^{50}$, A. Macchiolo ${ }^{103}$, C.M. Macdonald ${ }^{141}$, B. Maček ${ }^{78}$, J. Machado Miguens ${ }^{124,128 b}$, D. Madaffari ${ }^{88}$, R. Madar ${ }^{37}$, W.F. Mader ${ }^{47}$, A. Madsen ${ }^{45}$, J. Maeda ${ }^{70}$, S. Maeland ${ }^{15}$, T. Maeno ${ }^{27}$, A.S. Maevskiy ${ }^{101}$, E. Magradze ${ }^{57}$, J. Mahlstedt ${ }^{109}$, C. Maiani ${ }^{119}$, C. Maidantchik ${ }^{26 a}$, A.A. Maier ${ }^{103}$, T. Maier ${ }^{102}$, A. Maio ${ }^{128 a, 128 b, 128 d}$, O. Majersky ${ }^{146 a}$, S. Majewski ${ }^{118}$, Y. Makida $^{69}$, N. Makovec ${ }^{119}$, B. Malaescu ${ }^{83}$, Pa. Malecki ${ }^{42}$, V.P. Maleev ${ }^{125}$, F. Malek ${ }^{58}$, U. Mallik ${ }^{66}$, D. Malon ${ }^{6}$, C. Malone ${ }^{30}$, S. Maltezos ${ }^{10}$, S. Malyukov ${ }^{32}$, J. Mamuzic ${ }^{170}$, G. Mancini ${ }^{50}$, L. Mandelli ${ }^{94 a}$, I. Mandić ${ }^{78}$, J. Maneira ${ }^{128 a, 128 b}$, L. Manhaes de Andrade Filho ${ }^{26 b}$, J. Manjarres Ramos ${ }^{47}$, A. Mann ${ }^{102}$, A. Manousos ${ }^{32}$, B. Mansoulie ${ }^{138}$, J.D. Mansour ${ }^{35 a}$, R. Mantifel ${ }^{90}$, M. Mantoani ${ }^{57}$, S. Manzoni ${ }^{94 a, 94 b}$, L. Mapelli ${ }^{32}$, G. Marceca ${ }^{29}$, L. March ${ }^{52}$, L. Marchese ${ }^{122}$, G. Marchiori ${ }^{83}$, M. Marcisovsky ${ }^{129}$, M. Marjanovic ${ }^{37}$, D.E. Marley ${ }^{92}$, F. Marroquim ${ }^{26 a}$, S.P. Marsden ${ }^{87}$, Z. Marshall ${ }^{16}$, M.U.F Martensson ${ }^{168}$, S. Marti-Garcia ${ }^{170}$, C.B. Martin ${ }^{113}$, T.A. Martin ${ }^{173}$, V.J. Martin ${ }^{49}$, B. Martin dit Latour ${ }^{15}$, M. Martinez ${ }^{13, u}$, V.I. Martinez Outschoorn ${ }^{169}$, S. Martin-Haugh ${ }^{133}$, V.S. Martoiu ${ }^{28 b}$, A.C. Martyniuk ${ }^{81}$, A. Marzin ${ }^{32}$, L. Masetti ${ }^{86}$, T. Mashimo ${ }^{157}$, R. Mashinistov ${ }^{98}$, J. Masik ${ }^{87}$, A.L. Maslennikov ${ }^{111, c}$, L. Massa ${ }^{135 a, 135 b}$, 
P. Mastrandrea ${ }^{5}$, A. Mastroberardino ${ }^{40 a, 40 b}$, T. Masubuchi ${ }^{157}$, P. Mättig ${ }^{178}$, J. Maurer $^{28 b}$, S.J. Maxfield ${ }^{77}$, D.A. Maximov ${ }^{111, c}$, R. Mazini ${ }^{153}$, I. Maznas ${ }^{156}$, S.M. Mazza ${ }^{94 a, 94 b}$, N.C. Mc Fadden ${ }^{107}$, G. Mc Goldrick ${ }^{161}$, S.P. Mc Kee ${ }^{92}$, A. McCarn ${ }^{92}$, R.L. McCarthy ${ }^{150}$, T.G. McCarthy ${ }^{103}$, L.I. McClymont ${ }^{81}$, E.F. McDonald ${ }^{91}$, J.A. Mcfayden ${ }^{81}$, G. Mchedlidze ${ }^{57}$, S.J. McMahon ${ }^{133}$, P.C. McNamara ${ }^{91}$, R.A. McPherson ${ }^{172, o}$, S. Meehan ${ }^{140}$, T.J. Megy ${ }^{51}$, S. Mehlhase ${ }^{102}$, A. Mehta ${ }^{77}$, T. Meideck ${ }^{58}$, K. Meier ${ }^{60 a}$, B. Meirose ${ }^{44}$, D. Melini ${ }^{170, a h}$, B.R. Mellado Garcia ${ }^{147 c}$, J.D. Mellenthin ${ }^{57}$, M. Melo ${ }^{146 a}$, F. Meloni ${ }^{18}$, S.B. Menary ${ }^{87}$, L. Meng ${ }^{77}$, X.T. Meng ${ }^{92}$, A. Mengarelli22a,22b, S. Menke ${ }^{103}$, E. Meoni ${ }^{40 a, 40 b}$, S. Mergelmeyer ${ }^{17}$, P. Mermod ${ }^{52}$, L. Merola ${ }^{106 a, 106 b}$, C. Meroni ${ }^{94 a}$, F.S. Merritt ${ }^{33}$, A. Messina ${ }^{134 a, 134 b}$, J. Metcalfe ${ }^{6}$, A.S. Mete ${ }^{166}$, C. Meyer ${ }^{124}$, J-P. Meyer ${ }^{138}$, J. Meyer ${ }^{109}$, H. Meyer Zu Theenhausen ${ }^{60 a}$, F. Miano ${ }^{151}$, R.P. Middleton ${ }^{133}$, S. Miglioranzi ${ }^{53 a, 53 b}$, L. Mijović ${ }^{49}$, G. Mikenberg ${ }^{175}$, M. Mikestikova ${ }^{129}$,

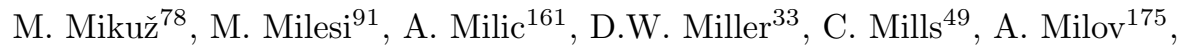

D.A. Milstead ${ }^{148 a, 148 b}$, A.A. Minaenko ${ }^{132}$, Y. Minami ${ }^{157}$, I.A. Minashvili68, A.I. Mincer ${ }^{112}$, B. Mindur ${ }^{41 a}$, M. Mineev ${ }^{68}$, Y. Minegishi ${ }^{157}$, Y. Ming ${ }^{176}$, L.M. Mir ${ }^{13}$, K.P. Mistry ${ }^{124}$, T. Mitani ${ }^{174}$, J. Mitrevski102 ${ }^{102}$ V.A. Mitsou ${ }^{170}$, A. Miucci ${ }^{18}$, P.S. Miyagawa ${ }^{141}$, A. Mizukami ${ }^{69}$, J.U. Mjörnmark ${ }^{84}$, T. Mkrtchyan ${ }^{180}$, M. Mlynarikova ${ }^{131}$, T. Moa ${ }^{148 a, 148 b}$, K. Mochizuki ${ }^{97}$, P. $\operatorname{Mogg}^{51}$, S. Mohapatra ${ }^{38}$, S. Molander ${ }^{148 a, 148 b}$, R. Moles-Valls ${ }^{23}$, R. Monden ${ }^{71}$, M.C. Mondragon ${ }^{93}$, K. Mönig ${ }^{45}$, J. Monk ${ }^{39}$, E. Monnier ${ }^{88}$, A. Montalbano ${ }^{150}$, J. Montejo Berlingen ${ }^{32}$, F. Monticelli ${ }^{74}$, S. Monzani ${ }^{94 a, 94 b}$, R.W. Moore ${ }^{3}$, N. Morange ${ }^{119}$, D. Moreno ${ }^{21}$, M. Moreno Llácer ${ }^{32}$, P. Morettini ${ }^{53 a}$, S. Morgenstern ${ }^{32}$, D. Mori ${ }^{144}$, T. Mori ${ }^{157}$, M. Morii ${ }^{59}$, M. Morinaga ${ }^{157}$, V. Morisbak ${ }^{121}$, A.K. Morley ${ }^{152}$, G. Mornacchi ${ }^{32}$, J.D. Morris ${ }^{79}$, L. Morvaj ${ }^{150}$, P. Moschovakos ${ }^{10}$, M. Mosidze ${ }^{54 b}$, H.J. Moss ${ }^{141}$, J. Moss ${ }^{145, a i}$, K. Motohashi ${ }^{159}$, R. Mount ${ }^{145}$, E. Mountricha ${ }^{27}$, E.J.W. Moyse $^{89}$, S. Muanza ${ }^{88}$, R.D. Mudd ${ }^{19}$, F. Mueller ${ }^{103}$, J. Mueller ${ }^{127}$, R.S.P. Mueller ${ }^{102}$, D. Muenstermann ${ }^{75}$, P. Mullen ${ }^{56}$, G.A. Mullier ${ }^{18}$, F.J. Munoz Sanchez ${ }^{87}$, W.J. Murray ${ }^{173,133}$, H. Musheghyan ${ }^{181}$, M. Muškinja ${ }^{78}$, A.G. Myagkov ${ }^{132, a j}$, M. Myska ${ }^{130}$, B.P. Nachman ${ }^{16}$, O. Nackenhorst ${ }^{52}$, K. Nagai ${ }^{122}$,

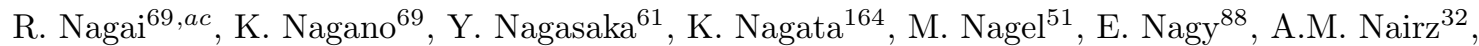
Y. Nakahama ${ }^{105}$, K. Nakamura ${ }^{69}$, T. Nakamura ${ }^{157}$, I. Nakano ${ }^{114}$, R.F. Naranjo Garcia ${ }^{45}$, R. Narayan ${ }^{11}$, D.I. Narrias Villar ${ }^{60 a}$, I. Naryshkin ${ }^{125}$, T. Naumann ${ }^{45}$, G. Navarro ${ }^{21}$, R. Nayyar ${ }^{7}$, H.A. Neal ${ }^{92}$, P.Yu. Nechaeva ${ }^{98}$, T.J. Neep ${ }^{138}$, A. Negri ${ }^{123 a, 123 b}$, M. Negrini ${ }^{22 a}$, S. Nektarijevic ${ }^{108}$, C. Nellist ${ }^{119}$, A. Nelson ${ }^{166}$, M.E. Nelson ${ }^{122}$, S. Nemecek ${ }^{129}$, P. Nemethy ${ }^{112}$, M. Nessi ${ }^{32, a k}$, M.S. Neubauer ${ }^{169}$, M. Neumann ${ }^{178}$, P.R. Newman ${ }^{19}$, T.Y. Ng ${ }^{62 c}$, T. Nguyen Manh ${ }^{97}$, R.B. Nickerson ${ }^{122}$, R. Nicolaidou ${ }^{138}$, J. Nielsen ${ }^{139}$, V. Nikolaenko ${ }^{132, a j}$, I. Nikolic-Audit ${ }^{83}$, K. Nikolopoulos ${ }^{19}$, J.K. Nilsen ${ }^{121}$, P. Nilsson ${ }^{27}$, Y. Ninomiya ${ }^{157}$, A. Nisati ${ }^{134 a}$, N. Nishu ${ }^{35 c}$, R. Nisius ${ }^{103}$, I. Nitsche ${ }^{46}$, T. Nobe ${ }^{157}$, Y. Noguchi ${ }^{71}$, M. Nomachi ${ }^{120}$, I. Nomidis ${ }^{31}$, M.A. Nomura ${ }^{27}$, T. Nooney ${ }^{79}$, M. Nordberg ${ }^{32}$, N. Norjoharuddeen ${ }^{122}$, O. Novgorodova ${ }^{47}$, S. Nowak ${ }^{103}$, M. Nozaki ${ }^{69}$, L. Nozka ${ }^{117}$, K. Ntekas ${ }^{166}$, E. Nurse ${ }^{81}$, F. Nuti ${ }^{91}$, K. O'connor ${ }^{25}$, D.C. O'Neil ${ }^{144}$, A.A. O'Rourke ${ }^{45}$, V. O'Shea ${ }^{56}$, F.G. Oakham ${ }^{31, d}$, H. Oberlack ${ }^{103}$,

T. Obermann ${ }^{23}$, J. Ocariz ${ }^{83}$, A. Ochi ${ }^{70}$, I. Ochoa ${ }^{38}$, J.P. Ochoa-Ricoux ${ }^{34 a}$, S. Oda ${ }^{73}$, S. Odaka $^{69}$, H. Ogren ${ }^{64}$, A. $\mathrm{Oh}^{87}$, S.H. Oh ${ }^{48}$, C.C. Ohm ${ }^{16}$, H. Ohman ${ }^{168}$, H. Oide ${ }^{53 a, 53 b}$, H. Okawa ${ }^{164}$, Y. Okumura ${ }^{157}$, T. Okuyama ${ }^{69}$, A. Olariu ${ }^{28 b}$, L.F. Oleiro Seabra ${ }^{128 a}$, S.A. Olivares Pino ${ }^{49}$, D. Oliveira Damazio ${ }^{27}$, A. Olszewski ${ }^{42}$, J. Olszowska ${ }^{42}$, A. Onofre ${ }^{128 a, 128 \mathrm{e}}$, K. Onogi ${ }^{105}$, P.U.E. Onyisi ${ }^{11, y}$, M.J. Oreglia ${ }^{33}$, Y. Oren ${ }^{155}$, D. Orestano ${ }^{136 a, 136 b}$, N. Orlando ${ }^{62 b}$, R.S. Orr ${ }^{161}$,

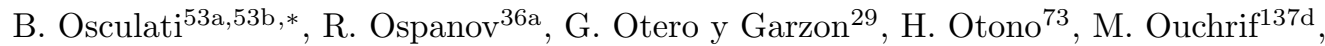
F. Ould-Saada ${ }^{121}$, A. Ouraou ${ }^{138}$, K.P. Oussoren ${ }^{109}$, Q. Ouyang ${ }^{35 a}$, M. Owen ${ }^{56}$, R.E. Owen ${ }^{19}$, V.E. Ozcan ${ }^{20 a}$, N. Ozturk ${ }^{8}$, K. Pachal ${ }^{144}$, A. Pacheco Pages ${ }^{13}$, L. Pacheco Rodriguez ${ }^{138}$, C. Padilla Aranda ${ }^{13}$, S. Pagan Griso ${ }^{16}$, M. Paganini ${ }^{179}$, F. Paige ${ }^{27}$, G. Palacino ${ }^{64}$, S. Palazzo ${ }^{40 a, 40 b}$, S. Palestini ${ }^{32}$, M. Palka ${ }^{41 b}$, D. Pallin ${ }^{37}$, E.St. Panagiotopoulou ${ }^{10}$, 
I. Panagoulias ${ }^{10}$, C.E. Pandini ${ }^{83}$, J.G. Panduro Vazquez ${ }^{80}$, P. Pani ${ }^{32}$, S. Panitkin ${ }^{27}$, D. Pantea ${ }^{28 b}$, L. Paolozzi ${ }^{52}$, Th.D. Papadopoulou ${ }^{10}$, K. Papageorgiou ${ }^{9}$, A. Paramonov ${ }^{6}$,

D. Paredes Hernandez ${ }^{179}$, A.J. Parker ${ }^{75}$, M.A. Parker ${ }^{30}$, K.A. Parker ${ }^{45}$, F. Parodi ${ }^{53 a, 53 b}$, J.A. Parsons ${ }^{38}$, U. Parzefall ${ }^{51}$, V.R. Pascuzzi ${ }^{161}$, J.M. Pasner ${ }^{139}$, E. Pasqualucci ${ }^{134 a}$, S. Passaggio ${ }^{53 a}$, Fr. Pastore ${ }^{80}$, S. Pataraia ${ }^{178}$, J.R. Pater ${ }^{87}$, T. Pauly ${ }^{32}$, B. Pearson ${ }^{103}$, S. Pedraza Lopez ${ }^{170}$, R. Pedro ${ }^{128 a, 128 b}$, S.V. Peleganchuk ${ }^{111, c}$, O. Penc ${ }^{129}$, C. Peng ${ }^{35 a}$, H. Peng ${ }^{36 a}$, J. Penwell ${ }^{64}$, B.S. Peralva ${ }^{26 b}$, M.M. Perego ${ }^{138}$, D.V. Perepelitsa ${ }^{27}$, L. Perini ${ }^{94 a, 94 b}$, H. Pernegger ${ }^{32}$, S. Perrella ${ }^{106 a, 106 b}$, R. Peschke ${ }^{45}$, V.D. Peshekhonov ${ }^{68, *}$, K. Peters ${ }^{45}$, R.F.Y. Peters ${ }^{87}$, B.A. Petersen ${ }^{32}$, T.C. Petersen ${ }^{39}$, E. Petit ${ }^{58}$, A. Petridis ${ }^{1}$, C. Petridou ${ }^{156}$, P. Petroff ${ }^{119}$, E. Petrolo ${ }^{134 a}$, M. Petrov ${ }^{122}$, F. Petrucci ${ }^{136 a, 136 b}$, N.E. Pettersson ${ }^{89}$, A. Peyaud ${ }^{138}$, R. Pezoa ${ }^{34 b}$, F.H. Phillips ${ }^{93}$, P.W. Phillips ${ }^{133}$, G. Piacquadio ${ }^{150}$, E. Pianori ${ }^{173}$, A. Picazio ${ }^{89}$, E. Piccaro ${ }^{79}$, M.A. Pickering ${ }^{122}$, R. Piegaia ${ }^{29}$, J.E. Pilcher ${ }^{33}$, A.D. Pilkington ${ }^{87}$, A.W.J. Pin ${ }^{87}$, M. Pinamonti 135a,135b, J.L. Pinfold ${ }^{3}$, H. Pirumov ${ }^{45}$, M. Pitt ${ }^{175}$, L. Plazak ${ }^{146 a}$, M.-A. Pleier ${ }^{27}$, V. Pleskot ${ }^{86}$, E. Plotnikova ${ }^{68}$, D. Pluth ${ }^{67}$, P. Podberezko $^{111}$, R. Poettgen ${ }^{148 a, 148 b}$, R. Poggi ${ }^{123 a, 123 b}$, L. Poggioli ${ }^{119}$, D. Pohl ${ }^{23}$, G. Polesello ${ }^{123 a}$, A. Poley ${ }^{45}$, A. Policicchio ${ }^{40 a, 40 b}$, R. Polifka ${ }^{32}$, A. Polini ${ }^{22 a}$, C.S. Pollard ${ }^{56}$, V. Polychronakos ${ }^{27}$, K. Pommès ${ }^{32}$, D. Ponomarenko ${ }^{100}$, L. Pontecorvo ${ }^{134 a}$, B.G. Pope ${ }^{93}$, G.A. Popeneciu ${ }^{28 d}$, A. Poppleton ${ }^{32}$, S. Pospisil ${ }^{130}$, K. Potamianos ${ }^{16}$, I.N. Potrap ${ }^{68}$, C.J. Potter ${ }^{30}$, G. Poulard ${ }^{32}$, T. Poulsen ${ }^{84}$, J. Poveda ${ }^{32}$, M.E. Pozo Astigarraga ${ }^{32}$, P. Pralavorio ${ }^{88}$, A. Pranko ${ }^{16}$, S. Prell ${ }^{67}$, D. Price ${ }^{87}$, L.E. Price ${ }^{6}$, M. Primavera ${ }^{76 a}$, S. Prince ${ }^{90}$, N. Proklova ${ }^{100}$, K. Prokofiev $^{62 c}$, F. Prokoshin ${ }^{34 b}$,

S. Protopopescu ${ }^{27}$, J. Proudfoot ${ }^{6}$, M. Przybycien ${ }^{41 a}$, A. Puri ${ }^{169}$, P. Puzo ${ }^{119}$, J. Qian ${ }^{92}$, G. Qin ${ }^{56}$, Y. Qin ${ }^{87}$, A. Quadt ${ }^{57}$, M. Queitsch-Maitland ${ }^{45}$, D. Quilty ${ }^{56}$, S. Raddum ${ }^{121}$, V. Radeka ${ }^{27}$, V. Radescu ${ }^{122}$, S.K. Radhakrishnan ${ }^{150}$, P. Radloff ${ }^{118}$, P. Rados ${ }^{91}$, F. Ragusa ${ }^{94 a, 94 b}$, G. Rahal ${ }^{182}$, J.A. Raine ${ }^{87}$, S. Rajagopalan ${ }^{27}$, C. Rangel-Smith ${ }^{168}$, T. Rashid ${ }^{119}$, S. Raspopov ${ }^{5}$, M.G. Ratti ${ }^{94 a, 94 b}$, D.M. Rauch ${ }^{45}$, F. Rauscher ${ }^{102}$, S. Rave ${ }^{86}$, I. Ravinovich ${ }^{175}$, J.H. Rawling ${ }^{87}$, M. Raymond ${ }^{32}$, A.L. Read ${ }^{121}$, N.P. Readioff ${ }^{58}$, M. Reale ${ }^{76 a, 76 b}$, D.M. Rebuzzi ${ }^{123 a, 123 b}$, A. Redelbach ${ }^{177}$, G. Redlinger ${ }^{27}$, R. Reece ${ }^{139}$, R.G. Reed ${ }^{147 c}$, K. Reeves ${ }^{44}$, L. Rehnisch $^{17}$, J. Reichert ${ }^{124}$, A. Reiss ${ }^{86}$, C. Rembser ${ }^{32}$, H. Ren $^{35 a}$, M. Rescigno ${ }^{134 a}$, S. Resconi ${ }^{94 a}$, E.D. Resseguie ${ }^{124}$, S. Rettie ${ }^{171}$, E. Reynolds ${ }^{19}$, O.L. Rezanova ${ }^{111, c}$, P. Reznicek ${ }^{131}$, R. Rezvani ${ }^{97}$, R. Richter ${ }^{103}$, S. Richter ${ }^{81}$, E. Richter-Was ${ }^{41 b}$, O. Ricken ${ }^{23}$, M. Ridel ${ }^{83}$, P. Rieck ${ }^{103}$, C.J. Riegel ${ }^{178}$, J. Rieger ${ }^{57}$, O. Rifki ${ }^{115}$, M. Rijssenbeek ${ }^{150}$, A. Rimoldi'123a,123b, M. Rimoldi ${ }^{18}$, L. Rinaldi ${ }^{22 a}$, G. Ripellino ${ }^{149}$, B. Ristić5 ${ }^{52}$, E. Ritsch ${ }^{32}$, I. Riu ${ }^{13}$, F. Rizatdinova ${ }^{116}$, E. Rizvi ${ }^{79}$, C. Rizzi ${ }^{13}$, R.T. Roberts ${ }^{87}$, S.H. Robertson ${ }^{90, o}$, A. Robichaud-Veronneau ${ }^{90}$, D. Robinson ${ }^{30}$, J.E.M. Robinson ${ }^{45}$, A. Robson ${ }^{56}$, E. Rocco $^{86}$, C. Roda ${ }^{126 a, 126 b}$, Y. Rodina ${ }^{88, a l}$,

S. Rodriguez Bosca ${ }^{170}$, A. Rodriguez Perez ${ }^{13}$, D. Rodriguez Rodriguez ${ }^{170}$, S. Roe ${ }^{32}$, C.S. Rogan ${ }^{59}$, O. Røhne ${ }^{121}$, J. Roloff ${ }^{59}$, A. Romaniouk ${ }^{100}$, M. Romano $22 \mathrm{a}, 22 \mathrm{~b}$, S.M. Romano Saez ${ }^{37}$, E. Romero Adam ${ }^{170}$, N. Rompotis ${ }^{77}$, M. Ronzani ${ }^{51}$, L. Roos ${ }^{83}$, S. Rosati ${ }^{134 a}$, K. Rosbach ${ }^{51}$, P. Rose ${ }^{139}$, N.-A. Rosien ${ }^{57}$, E. Rossi ${ }^{106 a, 106 b}$, L.P. Rossi ${ }^{53 a}$, J.H.N. Rosten ${ }^{30}$, R. Rosten ${ }^{140}$, M. Rotaru ${ }^{28 b}$, I. Roth ${ }^{175}$, J. Rothberg ${ }^{140}$, D. Rousseau ${ }^{119}$, A. Rozanov ${ }^{88}$, Y. Rozen ${ }^{154}$, X. Ruan ${ }^{147 c}$, F. Rubbo ${ }^{145}$, F. Rühr ${ }^{51}$, A. Ruiz-Martinez ${ }^{31}$, Z. Rurikova ${ }^{51}$, N.A. Rusakovich ${ }^{68}$, H.L. Russell ${ }^{90}$, J.P. Rutherfoord ${ }^{7}$, N. Ruthmann ${ }^{32}$, Y.F. Ryabov ${ }^{125}$, M. Rybar ${ }^{169}$, G. Rybkin ${ }^{119}$, S. Ryu ${ }^{6}$, A. Ryzhov ${ }^{132}$, G.F. Rzehorz ${ }^{57}$, A.F. Saavedra ${ }^{152}$, G. Sabato ${ }^{109}$, S. Sacerdoti ${ }^{29}$, H.F-W. Sadrozinski ${ }^{139}$, R. Sadykov ${ }^{68}$, F. Safai Tehrani ${ }^{134 a}$, P. Saha ${ }^{110}$, M. Sahinsoy ${ }^{60 a}$, M. Saimpert ${ }^{45}$, M. Saito ${ }^{157}$, T. Saito ${ }^{157}$, H. Sakamoto ${ }^{157}$, Y. Sakurai ${ }^{174}$, G. Salamanna ${ }^{136 a, 136 b}$, J.E. Salazar Loyola ${ }^{34 b}$, D. Salek ${ }^{109}$, P.H. Sales De Bruin ${ }^{168}$, D. Salihagic ${ }^{103}$, A. Salnikov ${ }^{145}$, J. Salt ${ }^{170}$, D. Salvatore ${ }^{40 a, 40 b}$, F. Salvatore ${ }^{151}$, A. Salvucci ${ }^{62 a, 62 b, 62 c}$, A. Salzburger ${ }^{32}$, D. Sammel ${ }^{51}$, D. Sampsonidis ${ }^{156}$, D. Sampsonidou ${ }^{156}$, J. Sánchez ${ }^{170}$, V. Sanchez Martinez ${ }^{170}$, A. Sanchez Pineda ${ }^{167 a, 167 c}$, H. Sandaker ${ }^{121}$, R.L. Sandbach ${ }^{79}$, C.O. Sander ${ }^{45}$, M. Sandhoff ${ }^{178}$, 
C. Sandoval ${ }^{21}$, D.P.C. Sankey ${ }^{133}$, M. Sannino ${ }^{53 a, 53 b}$, A. Sansoni ${ }^{50}$, C. Santoni ${ }^{37}$,

R. Santonico ${ }^{135 a, 135 b}$, H. Santos ${ }^{128 a}$, I. Santoyo Castillo ${ }^{151}$, A. Sapronov ${ }^{68}$, J.G. Saraiva ${ }^{128 a, 128 d}$,

B. Sarrazin ${ }^{23}$, O. Sasaki ${ }^{69}$, K. Sato ${ }^{164}$, E. Sauvan ${ }^{5}$, G. Savage ${ }^{80}$, P. Savard ${ }^{161, d}$, N. Savic ${ }^{103}$,

C. Sawyer ${ }^{133}$, L. Sawyer ${ }^{82, t}$, J. Saxon ${ }^{33}$, C. Sbarra ${ }^{22 a}$, A. Sbrizzi ${ }^{22 a, 22 b}$, T. Scanlon ${ }^{81}$,

D.A. Scannicchio ${ }^{166}$, M. Scarcella ${ }^{152}$, V. Scarfone ${ }^{40 a, 40 b}$, J. Schaarschmidt ${ }^{140}$, P. Schacht ${ }^{103}$,

B.M. Schachtner ${ }^{102}$, D. Schaefer ${ }^{32}$, L. Schaefer ${ }^{124}$, R. Schaefer ${ }^{45}$, J. Schaeffer ${ }^{86}$, S. Schaepe ${ }^{23}$,

S. Schaetzel ${ }^{60 b}$, U. Schäfer ${ }^{86}$, A.C. Schaffer ${ }^{119}$, D. Schaile ${ }^{102}$, R.D. Schamberger ${ }^{150}$, V. Scharf ${ }^{60 a}$, V.A. Schegelsky ${ }^{125}$, D. Scheirich ${ }^{131}$, M. Schernau ${ }^{166}$, C. Schiavi ${ }^{53 a, 53 b}$, S. Schier ${ }^{139}$,

L.K. Schildgen ${ }^{23}$, C. Schillo ${ }^{51}$, M. Schioppa ${ }^{40 a, 40 b}$, S. Schlenker ${ }^{32}$, K.R. Schmidt-Sommerfeld ${ }^{103}$,

K. Schmieden ${ }^{32}$, C. Schmitt ${ }^{86}$, S. Schmitt ${ }^{45}$, S. Schmitz ${ }^{86}$, U. Schnoor ${ }^{51}$, L. Schoeffel ${ }^{138}$,

A. Schoening ${ }^{60 b}$, B.D. Schoenrock ${ }^{93}$, E. Schopf ${ }^{23}$, M. Schott ${ }^{86}$, J.F.P. Schouwenberg ${ }^{108}$,

J. Schovancova ${ }^{181}$, S. Schramm ${ }^{52}$, N. Schuh ${ }^{86}$, A. Schulte ${ }^{86}$, M.J. Schultens ${ }^{23}$,

H.-C. Schultz-Coulon ${ }^{60 a}$, H. Schulz ${ }^{17}$, M. Schumacher ${ }^{51}$, B.A. Schumm ${ }^{139}$, Ph. Schune ${ }^{138}$,

A. Schwartzman ${ }^{145}$, T.A. Schwarz ${ }^{92}$, H. Schweiger ${ }^{87}$, Ph. Schwemling ${ }^{138}$, R. Schwienhorst ${ }^{93}$,

J. Schwindling ${ }^{138}$, A. Sciandra ${ }^{23}$, G. Sciolla ${ }^{25}$, F. Scuri ${ }^{126 a, 126 b}$, F. Scutti ${ }^{91}$, J. Searcy ${ }^{92}$,

P. Seema ${ }^{23}$, S.C. Seidel ${ }^{107}$, A. Seiden ${ }^{139}$, J.M. Seixas ${ }^{26 a}$, G. Sekhniaidze ${ }^{106 a}$, K. Sekhon ${ }^{92}$,

S.J. Sekula ${ }^{43}$, N. Semprini-Cesari ${ }^{22 a, 22 b}$, S. Senkin ${ }^{37}$, C. Serfon ${ }^{121}$, L. Serin ${ }^{119}$, L. Serkin ${ }^{167 a, 167 b}$, M. Sessa ${ }^{136 a, 136 b}$, R. Seuster ${ }^{172}$, H. Severini ${ }^{115}$, T. Sfiligoj ${ }^{78}$, F. Sforza ${ }^{32}$, A. Sfyrla ${ }^{52}$,

E. Shabalina ${ }^{57}$, N.W. Shaikh148a,148b, L.Y. Shan ${ }^{35 a}$, R. Shang ${ }^{169}$, J.T. Shank ${ }^{24}$, M. Shapiro ${ }^{16}$,

P.B. Shatalov ${ }^{99}$, K. Shaw ${ }^{167 a, 167 b}$, S.M. Shaw ${ }^{87}$, A. Shcherbakova ${ }^{148 a, 148 b}$, C.Y. Shehu ${ }^{151}$,

Y. Shen ${ }^{115}$, N. Sherafati ${ }^{31}$, P. Sherwood ${ }^{81}$, L. Shi ${ }^{153, a m}$, S. Shimizu ${ }^{70}$, C.O. Shimmin ${ }^{179}$,

M. Shimojima ${ }^{104}$, I.P.J. Shipsey ${ }^{122}$, S. Shirabe ${ }^{73}$, M. Shiyakova ${ }^{68, a n}$, J. Shlomi ${ }^{175}$, A. Shmeleva ${ }^{98}$,

D. Shoaleh Saadi ${ }^{97}$, M.J. Shochet ${ }^{33}$, S. Shojaii ${ }^{94 a}$, D.R. Shope ${ }^{115}$, S. Shrestha ${ }^{113}$, E. Shulga ${ }^{100}$,

M.A. Shupe ${ }^{7}$, P. Sicho ${ }^{129}$, A.M. Sickles ${ }^{169}$, P.E. Sidebo ${ }^{149}$, E. Sideras Haddad ${ }^{147 c}$,

O. Sidiropoulou ${ }^{177}$, A. Sidoti ${ }^{22 a, 22 b}$, F. Siegert ${ }^{47}$, Dj. Sijacki ${ }^{14}$, J. Silva ${ }^{128 a, 128 d}$,

S.B. Silverstein ${ }^{148 a}$, V. Simak ${ }^{130}$, Lj. Simic $^{14}$, S. Simion ${ }^{119}$, E. Simioni ${ }^{86}$, B. Simmons ${ }^{81}$,

M. Simon ${ }^{86}$, P. Sinervo ${ }^{161}$, N.B. Sinev ${ }^{118}$, M. Sioli ${ }^{22 a, 22 b}$, G. Siragusa ${ }^{177}$, I. Siral ${ }^{92}$,

S.Yu. Sivoklokov ${ }^{101}$, J. Sjölin ${ }^{148 a, 148 b}$, M.B. Skinner ${ }^{75}$, P. Skubic ${ }^{115}$, M. Slater ${ }^{19}$, T. Slavicek ${ }^{130}$,

M. Slawinska ${ }^{42}$, K. Sliwa ${ }^{165}$, R. Slovak ${ }^{131}$, V. Smakhtin ${ }^{175}$, B.H. Smart ${ }^{5}$, J. Smiesko ${ }^{146 a}$,

N. Smirnov ${ }^{100}$, S.Yu. Smirnov ${ }^{100}$, Y. Smirnov ${ }^{100}$, L.N. Smirnova ${ }^{101, a o}$, O. Smirnova ${ }^{84}$,

J.W. Smith ${ }^{57}$, M.N.K. Smith ${ }^{38}$, R.W. Smith ${ }^{38}$, M. Smizanska ${ }^{75}$, K. Smolek ${ }^{130}$, A.A. Snesarev ${ }^{98}$,

I.M. Snyder ${ }^{118}$, S. Snyder ${ }^{27}$, R. Sobie ${ }^{172, o}$, F. Socher ${ }^{47}$, A. Soffer ${ }^{155}$, D.A. Soh ${ }^{153}$,

G. Sokhrannyi ${ }^{78}$, C.A. Solans Sanchez ${ }^{32}$, M. Solar ${ }^{130}$, E.Yu. Soldatov ${ }^{100}$, U. Soldevila ${ }^{170}$,

A.A. Solodkov ${ }^{132}$, A. Soloshenko ${ }^{68}$, O.V. Solovyanov ${ }^{132}$, V. Solovyev ${ }^{125}$, P. Sommer ${ }^{51}$, H. Son ${ }^{165}$,

A. Sopczak ${ }^{130}$, D. Sosa ${ }^{60 b}$, C.L. Sotiropoulou ${ }^{126 a, 126 b}$, R. Soualah ${ }^{167 a, 167 c}$, A.M. Soukharev ${ }^{111, c}$,

D. South ${ }^{45}$, B.C. Sowden ${ }^{80}$, S. Spagnolo ${ }^{76 a, 76 b}$, M. Spalla ${ }^{126 a, 126 b}$, M. Spangenberg ${ }^{173}$,

F. Spanò ${ }^{80}$, D. Sperlich ${ }^{17}$, F. Spettel ${ }^{103}$, T.M. Spieker ${ }^{60 a}$, R. Spighi ${ }^{22 a}$, G. Spigo ${ }^{32}$, L.A. Spiller ${ }^{91}$,

M. Spousta ${ }^{131}$, R.D. St. Denis ${ }^{56, *}$, A. Stabile ${ }^{94 a}$, R. Stamen ${ }^{60 a}$, S. Stamm ${ }^{17}$, E. Stanecka ${ }^{42}$,

R.W. Stanek ${ }^{6}$, C. Stanescu ${ }^{136 a}$, M.M. Stanitzki ${ }^{45}$, B.S. Stapf ${ }^{109}$, S. Stapnes ${ }^{121}$,

E.A. Starchenko ${ }^{132}$, G.H. Stark ${ }^{33}$, J. Stark $^{58}$, S.H Stark ${ }^{39}$, P. Staroba ${ }^{129}$, P. Starovoitov ${ }^{60 a}$,

S. Stärz ${ }^{32}$, R. Staszewski ${ }^{42}$, P. Steinberg ${ }^{27}$, B. Stelzer ${ }^{144}$, H.J. Stelzer ${ }^{32}$, O. Stelzer-Chilton ${ }^{163 a}$,

H. Stenzel ${ }^{55}$, G.A. Stewart ${ }^{56}$, M.C. Stockton ${ }^{118}$, M. Stoebe ${ }^{90}$, G. Stoicea ${ }^{28 b}$, P. Stolte ${ }^{57}$,

S. Stonjek ${ }^{103}$, A.R. Stradling ${ }^{8}$, A. Straessner ${ }^{47}$, M.E. Stramaglia ${ }^{18}$, J. Strandberg ${ }^{149}$,

S. Strandberg ${ }^{148 a, 148 b}$, M. Strauss ${ }^{115}$, P. Strizenec ${ }^{146 b}$, R. Ströhmer ${ }^{177}$, D.M. Strom ${ }^{118}$,

R. Stroynowski ${ }^{43}$, A. Strubig ${ }^{108}$, S.A. Stucci ${ }^{27}$, B. Stugu ${ }^{15}$, N.A. Styles ${ }^{45}$, D. Su ${ }^{145}$, J. Su$^{127}$,

S. Suchek ${ }^{60 a}$, Y. Sugaya ${ }^{120}$, M. Suk ${ }^{130}$, V.V. Sulin ${ }^{98}$, DMS Sultan ${ }^{162 a, 162 b}$, S. Sultansoy ${ }^{4 c}$,

T. Sumida ${ }^{71}$, S. Sun ${ }^{59}$, X. Sun ${ }^{3}$, K. Suruliz ${ }^{151}$, C.J.E. Suster ${ }^{152}$, M.R. Sutton ${ }^{151}$, S. Suzuki ${ }^{69}$,

M. Svatos ${ }^{129}$, M. Swiatlowski ${ }^{33}$, S.P. Swift ${ }^{2}$, I. Sykora ${ }^{146 a}$, T. Sykora ${ }^{131}$, D. Ta ${ }^{51}$, K. Tackmann ${ }^{45}$, 


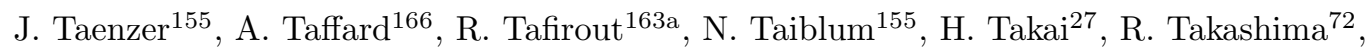
E.H. Takasugi ${ }^{103}$, T. Takeshita ${ }^{142}$, Y. Takubo ${ }^{69}$, M. Talby ${ }^{88}$, A.A. Talyshev ${ }^{111, c}$, J. Tanaka ${ }^{157}$, M. Tanaka ${ }^{159}$, R. Tanaka ${ }^{119}$, S. Tanaka ${ }^{69}$, R. Tanioka ${ }^{70}$, B.B. Tannenwald ${ }^{113}$, S. Tapia Araya ${ }^{34 b}$, S. Tapprogge ${ }^{86}$, S. Tarem ${ }^{154}$, G.F. Tartarelli ${ }^{94 a}$, P. Tas ${ }^{131}$, M. Tasevsky ${ }^{129}$, T. Tashiro ${ }^{71}$, E. Tassi ${ }^{40 a, 40 b}$, A. Tavares Delgado ${ }^{128 a, 128 b}$, Y. Tayalati ${ }^{137 e}$, A.C. Taylor ${ }^{107}$, G.N. Taylor ${ }^{91}$, P.T.E. Taylor ${ }^{91}$, W. Taylor ${ }^{163 b}$, P. Teixeira-Dias ${ }^{80}$, D. Temple ${ }^{144}$, H. Ten Kate $^{32}$, P.K. Teng ${ }^{153}$,

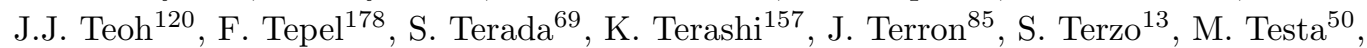
R.J. Teuscher ${ }^{161, o}$, T. Theveneaux-Pelzer ${ }^{88}$, J.P. Thomas ${ }^{19}$, J. Thomas-Wilsker ${ }^{80}$, P.D. Thompson ${ }^{19}$, A.S. Thompson ${ }^{56}$, L.A. Thomsen ${ }^{179}$, E. Thomson ${ }^{124}$, M.J. Tibbetts ${ }^{16}$, R.E. Ticse Torres ${ }^{88}$, V.O. Tikhomirov ${ }^{98, a p}$, Yu.A. Tikhonov ${ }^{111, c}$, S. Timoshenko ${ }^{100}$, P. Tipton ${ }^{179}$, S. Tisserant ${ }^{88}$, K. Todome ${ }^{159}$, S. Todorova-Nova ${ }^{5}$, J. Tojo ${ }^{73}$, S. Tokár ${ }^{146 a}$, K. Tokushuku ${ }^{69}$, E. Tolley ${ }^{59}$, L. Tomlinson ${ }^{87}$, M. Tomoto ${ }^{105}$, L. Tompkins ${ }^{145, a q}$, K. Toms ${ }^{107}$, B. Tong ${ }^{59}$,

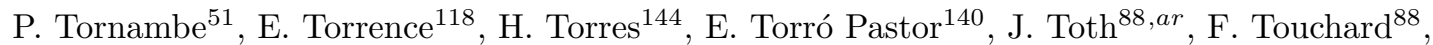

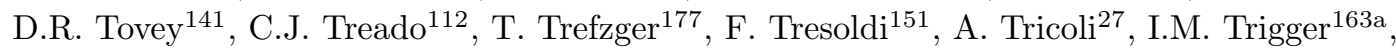
S. Trincaz-Duvoid ${ }^{83}$, M.F. Tripiana ${ }^{13}$, W. Trischuk ${ }^{161}$, B. Trocmé ${ }^{58}$, A. Trofymov ${ }^{45}$, C. Troncon ${ }^{94 a}$, M. Trottier-McDonald ${ }^{16}$, M. Trovatelli ${ }^{172}$, L. Truong ${ }^{167 a, 167 c}$, M. Trzebinski ${ }^{42}$, A. Trzupek ${ }^{42}$, K.W. Tsang ${ }^{62 a}$, J.C-L. Tseng ${ }^{122}$, P.V. Tsiareshka ${ }^{95}$, G. Tsipolitis ${ }^{10}$,

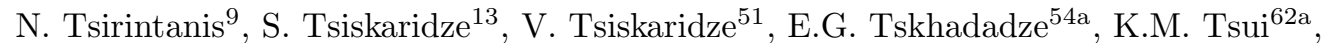

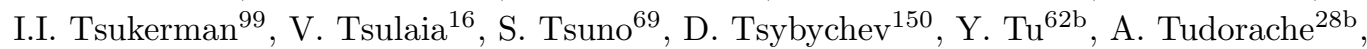
V. Tudorache ${ }^{28 b}$, T.T. Tulbure ${ }^{28 a}$, A.N. Tuna ${ }^{59}$, S.A. Tupputi ${ }^{22 a, 22 b}$, S. Turchikhin ${ }^{68}$, D. Turgeman ${ }^{175}$, I. Turk Cakir ${ }^{4 b, a s}$, R. Turra ${ }^{94 a, 94 b}$, P.M. Tuts ${ }^{38}$, G. Ucchielli ${ }^{22 a, 22 b}$, I. Ueda ${ }^{69}$, M. Ughetto ${ }^{148 a, 148 b}$, F. Ukegawa ${ }^{164}$, G. Unal ${ }^{32}$, A. Undrus ${ }^{27}$, G. Unel ${ }^{166}$, F.C. Ungaro ${ }^{91}$, Y. Unno ${ }^{69}$, C. Unverdorben ${ }^{102}$, J. Urban ${ }^{146 b}$, P. Urquijo ${ }^{91}$, P. Urrejola ${ }^{86}$, G. Usai ${ }^{8}$, J. Usui ${ }^{69}$, L. Vacavant ${ }^{88}$, V. Vacek ${ }^{130}$, B. Vachon ${ }^{90}$, C. Valderanis ${ }^{102}$, E. Valdes Santurio ${ }^{148 a, 148 b}$, S. Valentinetti ${ }^{22 \mathrm{a}, 22 \mathrm{~b}}$, A. Valero ${ }^{170}$, L. Valéry ${ }^{13}$, S. Valkar ${ }^{131}$, A. Vallier ${ }^{5}$, J.A. Valls Ferrer ${ }^{170}$, W. Van Den Wollenberg ${ }^{109}$, H. van der Graaf ${ }^{109}$, P. van Gemmeren ${ }^{6}$, J. Van Nieuwkoop ${ }^{144}$, I. van Vulpen ${ }^{109}$, M.C. van Woerden ${ }^{109}$, M. Vanadia ${ }^{135 a, 135 b}$, W. Vandelli ${ }^{32}$, A. Vaniachine ${ }^{160}$,

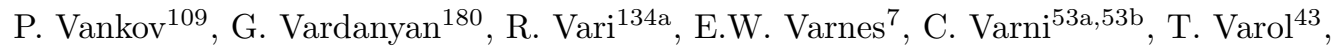

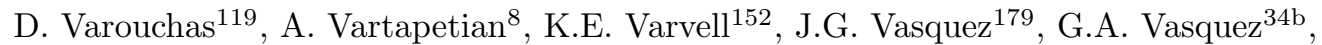
F. Vazeille ${ }^{37}$, T. Vazquez Schroeder ${ }^{90}$, J. Veatch ${ }^{57}$, V. Veeraraghavan ${ }^{7}$, L.M. Veloce ${ }^{161}$, F. Veloso ${ }^{128 a, 128 c}$, S. Veneziano ${ }^{134 a}$, A. Ventura ${ }^{76 a, 76 b}$, M. Venturi ${ }^{172}$, N. Venturi ${ }^{32}$,

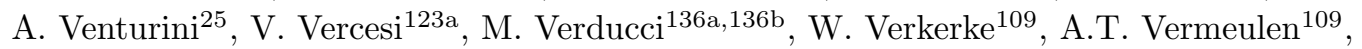
J.C. Vermeulen ${ }^{109}$, M.C. Vetterli ${ }^{144, d}$, N. Viaux Maira ${ }^{34 b}$, O. Viazlo ${ }^{84}$, I. Vichou ${ }^{169, *}$, T. Vickey ${ }^{141}$, O.E. Vickey Boeriu ${ }^{141}$, G.H.A. Viehhauser ${ }^{122}$, S. Viel ${ }^{16}$, L. Vigani $^{122}$,

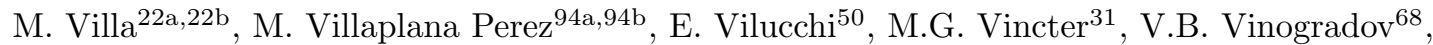
A. Vishwakarma ${ }^{45}$, C. Vittori ${ }^{22 a, 22 b}$, I. Vivarelli ${ }^{151}$, S. Vlachos ${ }^{10}$, M. Vlasak ${ }^{130}$, M. Vogel $^{178}$, P. Vokac ${ }^{130}$, G. Volpi ${ }^{126 a, 126 b}$, H. von der $\operatorname{Schmitt}^{103}$, E. von Toerne ${ }^{23}$, V. Vorobel ${ }^{131}$, K. Vorobev ${ }^{100}$, M. $\operatorname{Vos}^{170}$, R. Voss $^{32}$, J.H. Vossebeld ${ }^{77}$, N. Vranjes ${ }^{14}$, M. Vranjes Milosavljevic ${ }^{14}$, V. Vrba ${ }^{130}$, M. Vreeswijk ${ }^{109}$, R. Vuillermet ${ }^{32}$, I. Vukotic ${ }^{33}$, P. Wagner ${ }^{23}$, W. Wagner ${ }^{178}$, J. Wagner-Kuhr ${ }^{102}$, H. Wahlberg ${ }^{74}$, S. Wahrmund ${ }^{47}$, J. Wakabayashi ${ }^{105}$, J. Walder ${ }^{75}$, R. Walker ${ }^{102}$, W. Walkowiak ${ }^{143}$, V. Wallangen ${ }^{148 a, 148 b}$, C. Wang ${ }^{35 b}$, C. Wang ${ }^{36 b, a t}$, F. Wang ${ }^{176}$,

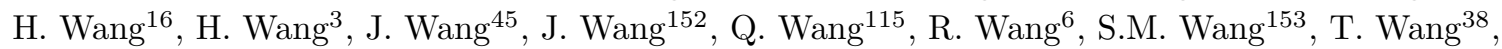
W. Wang ${ }^{153, a u}$, W. Wang ${ }^{36 a}$, Z. Wang ${ }^{36 c}$, C. Wanotayaroj ${ }^{118}$, A. Warburton ${ }^{90}$, C.P. Ward ${ }^{30}$, D.R. Wardrope ${ }^{81}$, A. Washbrook ${ }^{49}$, P.M. Watkins ${ }^{19}$, A.T. Watson ${ }^{19}$, M.F. Watson ${ }^{19}$, G. Watts ${ }^{140}$, S. Watts ${ }^{87}$, B.M. Waugh ${ }^{81}$, A.F. Webb ${ }^{11}$, S. Webb ${ }^{86}$, M.S. Weber ${ }^{18}$, S.W. Weber ${ }^{177}$, S.A. Weber ${ }^{31}$, J.S. Webster ${ }^{6}$, A.R. Weidberg ${ }^{122}$, B. Weinert ${ }^{64}$, J. Weingarten ${ }^{57}$, M. Weirich ${ }^{86}$, C. Weiser ${ }^{51}$, H. Weits ${ }^{109}$, P.S. Wells ${ }^{32}$, T. Wenaus ${ }^{27}$, T. Wengler ${ }^{32}$, S. Wenig ${ }^{32}$, N. Wermes ${ }^{23}$, M.D. Werner ${ }^{67}$, P. Werner ${ }^{32}$, M. Wessels ${ }^{60 a}$, K. Whalen ${ }^{118}$, N.L. Whallon ${ }^{140}$, A.M. Wharton ${ }^{75}$, 
A.S. White ${ }^{92}$, A. White ${ }^{8}$, M.J. White ${ }^{1}$, R. White ${ }^{34 b}$, D. Whiteson ${ }^{166}$, F.J. Wickens ${ }^{133}$ W. Wiedenmann ${ }^{176}$, M. Wielers ${ }^{133}$, C. Wiglesworth ${ }^{39}$, L.A.M. Wiik-Fuchs ${ }^{23}$, A. Wildauer ${ }^{103}$, F. Wilk ${ }^{87}$, H.G. Wilkens ${ }^{32}$, H.H. Williams ${ }^{124}$, S. Williams ${ }^{109}$, C. Willis ${ }^{93}$, S. Willocq ${ }^{89}$, J.A. Wilson ${ }^{19}$, I. Wingerter-Seez ${ }^{5}$, E. Winkels ${ }^{151}$, F. Winklmeier ${ }^{118}$, O.J. Winston ${ }^{151}$, B.T. Winter ${ }^{23}$, M. Wittgen ${ }^{145}$, M. Wobisch ${ }^{82, t}$, T.M.H. Wolf ${ }^{109}$, R. Wolff ${ }^{88}$, M.W. Wolter ${ }^{42}$, H. Wolters ${ }^{128 a}$,128c, V.W.S. Wong ${ }^{171}$, S.D. Worm ${ }^{19}$, B.K. Wosiek ${ }^{42}$, J. Wotschack ${ }^{32}$, K.W. Wozniak ${ }^{42}$, M. $\mathrm{Wu}^{33}$, S.L. $\mathrm{Wu}^{176}$, X. $\mathrm{Wu}^{52}$, Y. Wu ${ }^{92}$, T.R. Wyatt ${ }^{87}$, B.M. Wynne ${ }^{49}$, S. Xella ${ }^{39}$, Z. Xi ${ }^{92}$, L. Xia ${ }^{35 c}$, D. Xu ${ }^{35 a}$, L. Xu ${ }^{27}$, B. Yabsley ${ }^{152}$, S. Yacoob ${ }^{147 a}$, D. Yamaguchi ${ }^{159}$, Y. Yamaguchi ${ }^{120}$, A. Yamamoto ${ }^{69}$, S. Yamamoto ${ }^{157}$, T. Yamanaka ${ }^{157}$, M. Yamatani ${ }^{157}$,

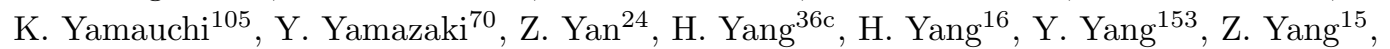
W-M. $\mathrm{Yao}^{16}$, Y.C. Yap ${ }^{83}$, Y. Yasu ${ }^{69}$, E. Yatsenko ${ }^{5}$, K.H. Yau Wong ${ }^{23}$, J. Ye $^{43}, \mathrm{~S} \mathrm{Ye}^{27}$, I. Yeletskikh ${ }^{68}$, E. Yigitbasi ${ }^{24}$, E. Yildirim ${ }^{86}$, K. Yorita ${ }^{174}$, K. Yoshihara ${ }^{124}$, C. Young ${ }^{145}$, C.J.S. Young ${ }^{32}$, J. $\mathrm{Yu}^{8}$, J. Yu ${ }^{67}$, S.P.Y. Yuen ${ }^{23}$, I. Yusuff ${ }^{30, a v}$, B. Zabinski ${ }^{42}$, G. Zacharis ${ }^{10}$, R. Zaidan ${ }^{13}$, A.M. Zaitsev ${ }^{132, a j}$, N. Zakharchuk ${ }^{45}$, J. Zalieckas ${ }^{15}$, A. Zaman ${ }^{150}$, S. Zambito ${ }^{59}$, D. Zanzi ${ }^{91}$, C. Zeitnitz ${ }^{178}$, A. Zemla ${ }^{41 a}$, J.C. Zeng ${ }^{169}$, Q. Zeng ${ }^{145}$, O. Zenin ${ }^{132}$, T. Ženišs ${ }^{146 a}$, D. Zerwas ${ }^{119}$, D. Zhang ${ }^{92}$, F. Zhang ${ }^{176}$, G. Zhang ${ }^{36 a, a w}$, H. Zhang ${ }^{35 b}$, J. Zhang ${ }^{6}$, L. Zhang ${ }^{51}$, L. Zhang ${ }^{36 a}$, M. Zhang ${ }^{169}$, P. Zhang ${ }^{35 b}$, R. Zhang ${ }^{23}$, R. Zhang ${ }^{36 a, a t}$, X. Zhang ${ }^{36 b}$, Y. Zhang ${ }^{35 a}$, Z. Zhang ${ }^{119}$, X. Zhao ${ }^{43}$, Y. Zhao ${ }^{36 b, a x}$, Z. Zhao ${ }^{36 a}$, A. Zhemchugov ${ }^{68}$, B. Zhou ${ }^{92}$, C. Zhou ${ }^{176}$, L. Zhou ${ }^{43}$, M. Zhou ${ }^{35 a}$, M. Zhou ${ }^{150}$, N. Zhou ${ }^{35 c}$, C.G. Zhu ${ }^{36 b}$, H. Zhu ${ }^{35 a}$, J. Zhu ${ }^{92}$, Y. Zhu ${ }^{36 a}$, X. Zhuang ${ }^{35 a}$, K. Zhukov ${ }^{98}$, A. Zibell ${ }^{177}$, D. Zieminska ${ }^{64}$, N.I. Zimine ${ }^{68}$, C. Zimmermann ${ }^{86}$, S. Zimmermann ${ }^{51}$, Z. Zinonos ${ }^{103}$, M. Zinser ${ }^{86}$, M. Ziolkowski ${ }^{143}$, L. Živković ${ }^{14}$, G. Zobernig ${ }^{176}$, A. Zoccoli ${ }^{22 a, 22 b}$, R. Zou ${ }^{33}$, M. zur Nedden ${ }^{17}$, L. Zwalinski ${ }^{32}$.

1 Department of Physics, University of Adelaide, Adelaide, Australia

2 Physics Department, SUNY Albany, Albany NY, United States of America

3 Department of Physics, University of Alberta, Edmonton AB, Canada

4 (a) Department of Physics, Ankara University, Ankara; ${ }^{(b)}$ Istanbul Aydin University, Istanbul; ${ }^{(c)}$ Division of Physics, TOBB University of Economics and Technology, Ankara, Turkey

5 LAPP, CNRS/IN2P3 and Université Savoie Mont Blanc, Annecy-le-Vieux, France

6 High Energy Physics Division, Argonne National Laboratory, Argonne IL, United States of America

7 Department of Physics, University of Arizona, Tucson AZ, United States of America

8 Department of Physics, The University of Texas at Arlington, Arlington TX, United States of America

9 Physics Department, National and Kapodistrian University of Athens, Athens, Greece

10 Physics Department, National Technical University of Athens, Zografou, Greece

11 Department of Physics, The University of Texas at Austin, Austin TX, United States of America

12 Institute of Physics, Azerbaijan Academy of Sciences, Baku, Azerbaijan

13 Institut de Física d'Altes Energies (IFAE), The Barcelona Institute of Science and Technology, Barcelona, Spain

14 Institute of Physics, University of Belgrade, Belgrade, Serbia

15 Department for Physics and Technology, University of Bergen, Bergen, Norway

16 Physics Division, Lawrence Berkeley National Laboratory and University of California, Berkeley CA, United States of America

17 Department of Physics, Humboldt University, Berlin, Germany

18 Albert Einstein Center for Fundamental Physics and Laboratory for High Energy Physics, University of Bern, Bern, Switzerland

19 School of Physics and Astronomy, University of Birmingham, Birmingham, United Kingdom

20 (a) Department of Physics, Bogazici University, Istanbul; ${ }^{(b)}$ Department of Physics Engineering, Gaziantep University, Gaziantep; ${ }^{(d)}$ Istanbul Bilgi University, Faculty of Engineering and Natural Sciences, Istanbul; ${ }^{(e)}$ Bahcesehir University, Faculty of Engineering and Natural Sciences, Istanbul, Turkey 
21 Centro de Investigaciones, Universidad Antonio Narino, Bogota, Colombia

22 (a) INFN Sezione di Bologna; ${ }^{(b)}$ Dipartimento di Fisica e Astronomia, Università di Bologna, Bologna, Italy

23 Physikalisches Institut, University of Bonn, Bonn, Germany

24 Department of Physics, Boston University, Boston MA, United States of America

25 Department of Physics, Brandeis University, Waltham MA, United States of America

26 (a) Universidade Federal do Rio De Janeiro COPPE/EE/IF, Rio de Janeiro; ${ }^{(b)}$ Electrical Circuits Department, Federal University of Juiz de Fora (UFJF), Juiz de Fora; ${ }^{(c)}$ Federal University of Sao Joao del Rei (UFSJ), Sao Joao del Rei; ${ }^{\left({ }^{d}\right)}$ Instituto de Fisica, Universidade de Sao Paulo, Sao Paulo, Brazil

27 Physics Department, Brookhaven National Laboratory, Upton NY, United States of America

28 (a) Transilvania University of Brasov, Brasov; ${ }^{(b)}$ Horia Hulubei National Institute of Physics and Nuclear Engineering, Bucharest; ${ }^{(c)}$ Department of Physics, Alexandru Ioan Cuza University of Iasi, Iasi; ${ }^{(d)}$ National Institute for Research and Development of Isotopic and Molecular Technologies, Physics Department, Cluj Napoca; ${ }^{(e)}$ University Politehnica Bucharest, Bucharest; (f) West University in Timisoara, Timisoara, Romania

29 Departamento de Física, Universidad de Buenos Aires, Buenos Aires, Argentina

30 Cavendish Laboratory, University of Cambridge, Cambridge, United Kingdom

31 Department of Physics, Carleton University, Ottawa ON, Canada

32 CERN, Geneva, Switzerland

33 Enrico Fermi Institute, University of Chicago, Chicago IL, United States of America

34 (a) Departamento de Física, Pontificia Universidad Católica de Chile, Santiago; ${ }^{(b)}$ Departamento de Física, Universidad Técnica Federico Santa María, Valparaíso, Chile

35 (a) Institute of High Energy Physics, Chinese Academy of Sciences, Beijing; ${ }^{(b)}$ Department of Physics, Nanjing University, Jiangsu; ${ }^{(c)}$ Physics Department, Tsinghua University, Beijing 100084, China

36 (a) Department of Modern Physics and State Key Laboratory of Particle Detection and Electronics, University of Science and Technology of China, Anhui; ${ }^{(b)}$ School of Physics, Shandong University, Shandong; (c) Department of Physics and Astronomy, Key Laboratory for Particle Physics, Astrophysics and Cosmology, Ministry of Education; Shanghai Key Laboratory for Particle Physics and Cosmology, Shanghai Jiao Tong University, Shanghai(also at PKU-CHEP);, China

37 Université Clermont Auvergne, CNRS/IN2P3, LPC, Clermont-Ferrand, France

38 Nevis Laboratory, Columbia University, Irvington NY, United States of America

39 Niels Bohr Institute, University of Copenhagen, Kobenhavn, Denmark

40 (a) INFN Gruppo Collegato di Cosenza, Laboratori Nazionali di Frascati; ${ }^{(b)}$ Dipartimento di Fisica, Università della Calabria, Rende, Italy

41 (a) AGH University of Science and Technology, Faculty of Physics and Applied Computer Science, Krakow; ${ }^{(b)}$ Marian Smoluchowski Institute of Physics, Jagiellonian University, Krakow, Poland

42 Institute of Nuclear Physics Polish Academy of Sciences, Krakow, Poland

43 Physics Department, Southern Methodist University, Dallas TX, United States of America

44 Physics Department, University of Texas at Dallas, Richardson TX, United States of America

45 DESY, Hamburg and Zeuthen, Germany

46 Lehrstuhl für Experimentelle Physik IV, Technische Universität Dortmund, Dortmund, Germany

47 Institut für Kern- und Teilchenphysik, Technische Universität Dresden, Dresden, Germany

48 Department of Physics, Duke University, Durham NC, United States of America

49 SUPA - School of Physics and Astronomy, University of Edinburgh, Edinburgh, United Kingdom

50 INFN Laboratori Nazionali di Frascati, Frascati, Italy

51 Fakultät für Mathematik und Physik, Albert-Ludwigs-Universität, Freiburg, Germany

52 Departement de Physique Nucleaire et Corpusculaire, Université de Genève, Geneva, Switzerland

53 (a) INFN Sezione di Genova; ${ }^{(b)}$ Dipartimento di Fisica, Università di Genova, Genova, Italy

54 (a) E. Andronikashvili Institute of Physics, Iv. Javakhishvili Tbilisi State University, Tbilisi; ${ }^{(b)}$ High Energy Physics Institute, Tbilisi State University, Tbilisi, Georgia 
56 SUPA - School of Physics and Astronomy, University of Glasgow, Glasgow, United Kingdom

57 II Physikalisches Institut, Georg-August-Universität, Göttingen, Germany

58 Laboratoire de Physique Subatomique et de Cosmologie, Université Grenoble-Alpes, CNRS/IN2P3, Grenoble, France

59 Laboratory for Particle Physics and Cosmology, Harvard University, Cambridge MA, United States of America

60 (a) Kirchhoff-Institut für Physik, Ruprecht-Karls-Universität Heidelberg, Heidelberg; ${ }^{(b)}$ Physikalisches Institut, Ruprecht-Karls-Universität Heidelberg, Heidelberg; (c) ZITI Institut für technische Informatik, Ruprecht-Karls-Universität Heidelberg, Mannheim, Germany

61 Faculty of Applied Information Science, Hiroshima Institute of Technology, Hiroshima, Japan

62 (a) Department of Physics, The Chinese University of Hong Kong, Shatin, N.T., Hong Kong; (b) Department of Physics, The University of Hong Kong, Hong Kong; (c) Department of Physics and Institute for Advanced Study, The Hong Kong University of Science and Technology, Clear Water Bay, Kowloon, Hong Kong, China

63 Department of Physics, National Tsing Hua University, Taiwan, Taiwan

64 Department of Physics, Indiana University, Bloomington IN, United States of America

65 Institut für Astro- und Teilchenphysik, Leopold-Franzens-Universität, Innsbruck, Austria

66 University of Iowa, Iowa City IA, United States of America

67 Department of Physics and Astronomy, Iowa State University, Ames IA, United States of America

68 Joint Institute for Nuclear Research, JINR Dubna, Dubna, Russia

69 KEK, High Energy Accelerator Research Organization, Tsukuba, Japan

70 Graduate School of Science, Kobe University, Kobe, Japan

71 Faculty of Science, Kyoto University, Kyoto, Japan

72 Kyoto University of Education, Kyoto, Japan

73 Research Center for Advanced Particle Physics and Department of Physics, Kyushu University, Fukuoka, Japan

74 Instituto de Física La Plata, Universidad Nacional de La Plata and CONICET, La Plata, Argentina

75 Physics Department, Lancaster University, Lancaster, United Kingdom

76 (a) INFN Sezione di Lecce; (b) Dipartimento di Matematica e Fisica, Università del Salento, Lecce, Italy

77 Oliver Lodge Laboratory, University of Liverpool, Liverpool, United Kingdom

78 Department of Experimental Particle Physics, Jožef Stefan Institute and Department of Physics, University of Ljubljana, Ljubljana, Slovenia

79 School of Physics and Astronomy, Queen Mary University of London, London, United Kingdom

80 Department of Physics, Royal Holloway University of London, Surrey, United Kingdom

81 Department of Physics and Astronomy, University College London, London, United Kingdom

82 Louisiana Tech University, Ruston LA, United States of America

83 Laboratoire de Physique Nucléaire et de Hautes Energies, UPMC and Université Paris-Diderot and CNRS/IN2P3, Paris, France

84 Fysiska institutionen, Lunds universitet, Lund, Sweden

85 Departamento de Fisica Teorica C-15, Universidad Autonoma de Madrid, Madrid, Spain

86 Institut für Physik, Universität Mainz, Mainz, Germany

87 School of Physics and Astronomy, University of Manchester, Manchester, United Kingdom

88 CPPM, Aix-Marseille Université and CNRS/IN2P3, Marseille, France

89 Department of Physics, University of Massachusetts, Amherst MA, United States of America

90 Department of Physics, McGill University, Montreal QC, Canada

91 School of Physics, University of Melbourne, Victoria, Australia

92 Department of Physics, The University of Michigan, Ann Arbor MI, United States of America

93 Department of Physics and Astronomy, Michigan State University, East Lansing MI, United States of America

94 (a) INFN Sezione di Milano; ${ }^{(b)}$ Dipartimento di Fisica, Università di Milano, Milano, Italy 
B.I. Stepanov Institute of Physics, National Academy of Sciences of Belarus, Minsk, Republic of Belarus

96 Research Institute for Nuclear Problems of Byelorussian State University, Minsk, Republic of Belarus

97 Group of Particle Physics, University of Montreal, Montreal QC, Canada

98 P.N. Lebedev Physical Institute of the Russian Academy of Sciences, Moscow, Russia

99 Institute for Theoretical and Experimental Physics (ITEP), Moscow, Russia

100 National Research Nuclear University MEPhI, Moscow, Russia

101 D.V. Skobeltsyn Institute of Nuclear Physics, M.V. Lomonosov Moscow State University, Moscow, Russia

102 Fakultät für Physik, Ludwig-Maximilians-Universität München, München, Germany

103 Max-Planck-Institut für Physik (Werner-Heisenberg-Institut), München, Germany

104 Nagasaki Institute of Applied Science, Nagasaki, Japan

105 Graduate School of Science and Kobayashi-Maskawa Institute, Nagoya University, Nagoya, Japan

106 (a) INFN Sezione di Napoli; ${ }^{(b)}$ Dipartimento di Fisica, Università di Napoli, Napoli, Italy

107 Department of Physics and Astronomy, University of New Mexico, Albuquerque NM, United States of America

108 Institute for Mathematics, Astrophysics and Particle Physics, Radboud University Nijmegen/Nikhef, Nijmegen, Netherlands

109 Nikhef National Institute for Subatomic Physics and University of Amsterdam, Amsterdam, Netherlands

110 Department of Physics, Northern Illinois University, DeKalb IL, United States of America

111 Budker Institute of Nuclear Physics, SB RAS, Novosibirsk, Russia

112 Department of Physics, New York University, New York NY, United States of America

113 Ohio State University, Columbus OH, United States of America

114 Faculty of Science, Okayama University, Okayama, Japan

115 Homer L. Dodge Department of Physics and Astronomy, University of Oklahoma, Norman OK, United States of America

116 Department of Physics, Oklahoma State University, Stillwater OK, United States of America

117 Palacký University, RCPTM, Olomouc, Czech Republic

118 Center for High Energy Physics, University of Oregon, Eugene OR, United States of America

119 LAL, Univ. Paris-Sud, CNRS/IN2P3, Université Paris-Saclay, Orsay, France

120 Graduate School of Science, Osaka University, Osaka, Japan

121 Department of Physics, University of Oslo, Oslo, Norway

122 Department of Physics, Oxford University, Oxford, United Kingdom

123 (a) INFN Sezione di Pavia; ${ }^{(b)}$ Dipartimento di Fisica, Università di Pavia, Pavia, Italy

124 Department of Physics, University of Pennsylvania, Philadelphia PA, United States of America

125 National Research Centre "Kurchatov Institute" B.P.Konstantinov Petersburg Nuclear Physics Institute, St. Petersburg, Russia

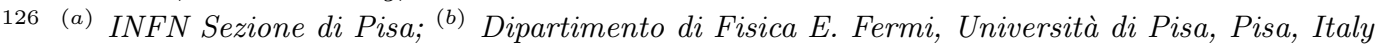

127 Department of Physics and Astronomy, University of Pittsburgh, Pittsburgh PA, United States of America

$128{ }^{(a)}$ Laboratório de InstrumentaC cão e Física Experimental de Partículas - LIP, Lisboa; ${ }^{(b)}$

Faculdade de Ciências, Universidade de Lisboa, Lisboa; ${ }^{(c)}$ Department of Physics, University of

Coimbra, Coimbra; ${ }^{\left({ }^{d}\right)}$ Centro de Física Nuclear da Universidade de Lisboa, Lisboa; ${ }^{(e)}$

Departamento de Fisica, Universidade do Minho, Braga; ${ }^{(f)}$ Departamento de Fisica Teorica y del

Cosmos and CAFPE, Universidad de Granada, Granada; ${ }^{(g)}$ Dep Fisica and CEFITEC of

Faculdade de Ciencias e Tecnologia, Universidade Nova de Lisboa, Caparica, Portugal

129 Institute of Physics, Academy of Sciences of the Czech Republic, Praha, Czech Republic

130 Czech Technical University in Prague, Praha, Czech Republic

131 Charles University, Faculty of Mathematics and Physics, Prague, Czech Republic

132 State Research Center Institute for High Energy Physics (Protvino), NRC KI, Russia 
133 Particle Physics Department, Rutherford Appleton Laboratory, Didcot, United Kingdom

134 (a) INFN Sezione di Roma; ${ }^{\left({ }^{b}\right)}$ Dipartimento di Fisica, Sapienza Università di Roma, Roma, Italy

135 (a) INFN Sezione di Roma Tor Vergata; ${ }^{(b)}$ Dipartimento di Fisica, Università di Roma Tor Vergata, Roma, Italy

136 (a) INFN Sezione di Roma Tre; ${ }^{(b)}$ Dipartimento di Matematica e Fisica, Università Roma Tre, Roma, Italy

137 (a) Faculté des Sciences Ain Chock, Réseau Universitaire de Physique des Hautes Energies Université Hassan II, Casablanca; ${ }^{(b)}$ Centre National de l'Energie des Sciences Techniques Nucleaires, Rabat; ${ }^{(c)}$ Faculté des Sciences Semlalia, Université Cadi Ayyad, LPHEA-Marrakech; (d) Faculté des Sciences, Université Mohamed Premier and LPTPM, Oujda; ${ }^{(e)}$ Faculté des sciences, Université Mohammed V, Rabat, Morocco

138 DSM/IRFU (Institut de Recherches sur les Lois Fondamentales de l'Univers), CEA Saclay (Commissariat à l'Energie Atomique et aux Energies Alternatives), Gif-sur-Yvette, France

139 Santa Cruz Institute for Particle Physics, University of California Santa Cruz, Santa Cruz CA, United States of America

140 Department of Physics, University of Washington, Seattle WA, United States of America

141 Department of Physics and Astronomy, University of Sheffield, Sheffield, United Kingdom

142 Department of Physics, Shinshu University, Nagano, Japan

143 Department Physik, Universität Siegen, Siegen, Germany

144 Department of Physics, Simon Fraser University, Burnaby BC, Canada

145 SLAC National Accelerator Laboratory, Stanford CA, United States of America

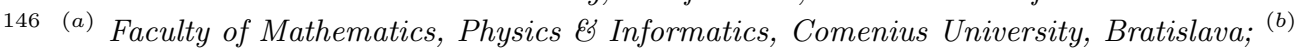

Department of Subnuclear Physics, Institute of Experimental Physics of the Slovak Academy of Sciences, Kosice, Slovak Republic

147 (a) Department of Physics, University of Cape Town, Cape Town; ${ }^{(b)}$ Department of Physics, University of Johannesburg, Johannesburg; ${ }^{(c)}$ School of Physics, University of the Witwatersrand, Johannesburg, South Africa

148 (a) Department of Physics, Stockholm University; ${ }^{(b)}$ The Oskar Klein Centre, Stockholm, Sweden

149 Physics Department, Royal Institute of Technology, Stockholm, Sweden

150 Departments of Physics $\&$ Astronomy and Chemistry, Stony Brook University, Stony Brook NY, United States of America

151 Department of Physics and Astronomy, University of Sussex, Brighton, United Kingdom

152 School of Physics, University of Sydney, Sydney, Australia

153 Institute of Physics, Academia Sinica, Taipei, Taiwan

154 Department of Physics, Technion: Israel Institute of Technology, Haifa, Israel

155 Raymond and Beverly Sackler School of Physics and Astronomy, Tel Aviv University, Tel Aviv, Israel

156 Department of Physics, Aristotle University of Thessaloniki, Thessaloniki, Greece

157 International Center for Elementary Particle Physics and Department of Physics, The University of Tokyo, Tokyo, Japan

158 Graduate School of Science and Technology, Tokyo Metropolitan University, Tokyo, Japan

159 Department of Physics, Tokyo Institute of Technology, Tokyo, Japan

160 Tomsk State University, Tomsk, Russia

161 Department of Physics, University of Toronto, Toronto ON, Canada

162 (a) INFN-TIFPA; ${ }^{(b)}$ University of Trento, Trento, Italy

163 (a) TRIUMF, Vancouver BC; ${ }^{(b)}$ Department of Physics and Astronomy, York University, Toronto ON, Canada

164 Faculty of Pure and Applied Sciences, and Center for Integrated Research in Fundamental Science and Engineering, University of Tsukuba, Tsukuba, Japan

165 Department of Physics and Astronomy, Tufts University, Medford MA, United States of America

166 Department of Physics and Astronomy, University of California Irvine, Irvine CA, United States of America 
(a) INFN Gruppo Collegato di Udine, Sezione di Trieste, Udine; ${ }^{(b)}$ ICTP, Trieste; ${ }^{(c)}$ Dipartimento di Chimica, Fisica e Ambiente, Università di Udine, Udine, Italy

168 Department of Physics and Astronomy, University of Uppsala, Uppsala, Sweden

169 Department of Physics, University of Illinois, Urbana IL, United States of America

170 Instituto de Fisica Corpuscular (IFIC) and Departamento de Fisica Atomica, Molecular y Nuclear and Departamento de Ingeniería Electrónica and Instituto de Microelectrónica de Barcelona (IMB-CNM), University of Valencia and CSIC, Valencia, Spain

171 Department of Physics, University of British Columbia, Vancouver BC, Canada

172 Department of Physics and Astronomy, University of Victoria, Victoria BC, Canada

173 Department of Physics, University of Warwick, Coventry, United Kingdom

174 Waseda University, Tokyo, Japan

175 Department of Particle Physics, The Weizmann Institute of Science, Rehovot, Israel

176 Department of Physics, University of Wisconsin, Madison WI, United States of America

177 Fakultät für Physik und Astronomie, Julius-Maximilians-Universität, Würzburg, Germany

178 Fakultät für Mathematik und Naturwissenschaften, Fachgruppe Physik, Bergische Universität Wuppertal, Wuppertal, Germany

179 Department of Physics, Yale University, New Haven CT, United States of America

180 Yerevan Physics Institute, Yerevan, Armenia

181 CH-1211 Geneva 23, Switzerland

182 Centre de Calcul de l'Institut National de Physique Nucléaire et de Physique des Particules (IN2P3), Villeurbanne, France

183 Academia Sinica Grid Computing, Institute of Physics, Academia Sinica, Taipei, Taiwan

a Also at Department of Physics, King's College London, London, United Kingdom

${ }^{b}$ Also at Institute of Physics, Azerbaijan Academy of Sciences, Baku, Azerbaijan

c Also at Novosibirsk State University, Novosibirsk, Russia

${ }^{d}$ Also at TRIUMF, Vancouver BC, Canada

e Also at Department of Physics $\mathcal{G}$ Astronomy, University of Louisville, Louisville, KY, United States of America

${ }^{f}$ Also at Physics Department, An-Najah National University, Nablus, Palestine

$g$ Also at Department of Physics, California State University, Fresno CA, United States of America

${ }^{h}$ Also at Department of Physics, University of Fribourg, Fribourg, Switzerland

i Also at II Physikalisches Institut, Georg-August-Universität, Göttingen, Germany

${ }^{j}$ Also at Departament de Fisica de la Universitat Autonoma de Barcelona, Barcelona, Spain

${ }^{k}$ Also at Departamento de Fisica e Astronomia, Faculdade de Ciencias, Universidade do Porto, Portugal

${ }^{l}$ Also at Tomsk State University, Tomsk, Russia

$m$ Also at The Collaborative Innovation Center of Quantum Matter (CICQM), Beijing, China

$n$ Also at Universita di Napoli Parthenope, Napoli, Italy

o Also at Institute of Particle Physics (IPP), Canada

${ }^{p}$ Also at Horia Hulubei National Institute of Physics and Nuclear Engineering, Bucharest, Romania

$q$ Also at Department of Physics, St. Petersburg State Polytechnical University, St. Petersburg, Russia

${ }^{r}$ Also at Borough of Manhattan Community College, City University of New York, New York City, United States of America

$s$ Also at Centre for High Performance Computing, CSIR Campus, Rosebank, Cape Town, South Africa

${ }^{t}$ Also at Louisiana Tech University, Ruston LA, United States of America

u Also at Institucio Catalana de Recerca i Estudis Avancats, ICREA, Barcelona, Spain

$v$ Also at Graduate School of Science, Osaka University, Osaka, Japan

w Also at Fakultät für Mathematik und Physik, Albert-Ludwigs-Universität, Freiburg, Germany

${ }^{x}$ Also at Institute for Mathematics, Astrophysics and Particle Physics, Radboud University Nijmegen/Nikhef, Nijmegen, Netherlands 
y Also at Department of Physics, The University of Texas at Austin, Austin TX, United States of America

$z$ Also at Institute of Theoretical Physics, Ilia State University, Tbilisi, Georgia

a Also at CERN, Geneva, Switzerland

ab Also at Georgian Technical University (GTU), Tbilisi, Georgia

ac Also at Ochadai Academic Production, Ochanomizu University, Tokyo, Japan

ad Also at Manhattan College, New York NY, United States of America

ae Also at Departamento de Física, Pontificia Universidad Católica de Chile, Santiago, Chile

af Also at Department of Physics, The University of Michigan, Ann Arbor MI, United States of America

ag Also at School of Physics, Shandong University, Shandong, China

ah Also at Departamento de Fisica Teorica y del Cosmos and CAFPE, Universidad de Granada, Granada, Portugal

ai Also at Department of Physics, California State University, Sacramento CA, United States of America

aj Also at Moscow Institute of Physics and Technology State University, Dolgoprudny, Russia

ak Also at Departement de Physique Nucleaire et Corpusculaire, Université de Genève, Geneva, Switzerland

al Also at Institut de Física d'Altes Energies (IFAE), The Barcelona Institute of Science and Technology, Barcelona, Spain

am Also at School of Physics, Sun Yat-sen University, Guangzhou, China

an Also at Institute for Nuclear Research and Nuclear Energy (INRNE) of the Bulgarian Academy of Sciences, Sofia, Bulgaria

ao Also at Faculty of Physics, M.V.Lomonosov Moscow State University, Moscow, Russia

ap Also at National Research Nuclear University MEPhI, Moscow, Russia

aq Also at Department of Physics, Stanford University, Stanford CA, United States of America

ar Also at Institute for Particle and Nuclear Physics, Wigner Research Centre for Physics, Budapest, Hungary

as Also at Giresun University, Faculty of Engineering, Turkey

at Also at CPPM, Aix-Marseille Université and CNRS/IN2P3, Marseille, France

au Also at Department of Physics, Nanjing University, Jiangsu, China

av Also at University of Malaya, Department of Physics, Kuala Lumpur, Malaysia

aw Also at Institute of Physics, Academia Sinica, Taipei, Taiwan

ax Also at LAL, Univ. Paris-Sud, CNRS/IN2P3, Université Paris-Saclay, Orsay, France

* Deceased 